Supporting information for:

\title{
Synthesis and stereoselective DNA binding abilities of new optically active open-chain polyamines
}

\author{
Carmen Peña, ${ }^{\mathrm{a}}$ Ignacio Alfonso, ${ }^{\mathrm{b},{ }^{*}}$ Blake Tooth, ${ }^{\mathrm{c}}$ Nicolas H. Voelcker ${ }^{\mathrm{c}}$ and \\ Vicente Gotor ${ }^{\mathrm{a},}$, \\ ${ }^{a}$ Departamento de Química Orgánica e Inorgánica, Facultad de Química, Universidad de Oviedo, Julián \\ Clavería, 8, E-33006, Oviedo (Spain) \\ ${ }^{b}$ Departamento de Química Inorgánica y Orgánica, ESTCE, Universidad Jaume I, Campus del Riu Sec, \\ Avenida Sos Baynat, s/n, E-12071, Castellón (Spain) \\ ${ }^{c}$ School of Chemistry, Physics and Earth Sciences, Flinders University of South Australia, Bedford Park, \\ South Australia, 5042 (Australia)
}

e-mail: ialfonso@qio.uji.es and vgs@fq.uniovi.es

Table of contents:

As the spectral data for both enantiomers of a given compound are identical, only copies of the NMR spectra for one enantiomer of every derivative are given.

General Techniques $\quad$ S3

Compounds characterization, for those not given in the manuscript $\quad \mathrm{S} 4$

${ }^{1} \mathrm{H}$ NMR spectrum of $(R, R)-\mathbf{3 a}\left(\mathrm{CDCl}_{3}, 300 \mathrm{MHz}\right) \quad \mathrm{S} 11$

${ }^{13} \mathrm{C}$ NMR spectrum of $(R, R)-3 \mathbf{3 a}\left(\mathrm{CDCl}_{3}, 75 \mathrm{MHz}\right) \quad \mathrm{S} 12$

DEPT spectrum of $(R, R)-\mathbf{3 a}\left(\mathrm{CDCl}_{3}, 75 \mathrm{MHz}\right) \quad \mathrm{S} 13$

$\begin{array}{ll}{ }^{1} \mathrm{H} \text { NMR spectrum of }(R, R) \mathbf{4 a}\left(\mathrm{CDCl}_{3}, 300 \mathrm{MHz}\right) & \mathrm{S} 14\end{array}$

${ }^{13} \mathrm{C}$ NMR spectrum of $(R, R)-\mathbf{4 a}\left(\mathrm{CDCl}_{3}, 75 \mathrm{MHz}\right) \quad \mathrm{S} 15$

DEPT spectrum of $(R, R)-\mathbf{4 a}\left(\mathrm{CDCl}_{3}, 75 \mathrm{MHz}\right) \quad \mathrm{S} 16$

$\begin{array}{ll}{ }^{1} \mathrm{H} \text { NMR spectrum of }(R, R)-\mathbf{5 a}\left(\mathrm{CDCl}_{3}, 300 \mathrm{MHz}\right) & \mathrm{S} 17\end{array}$

${ }^{13} \mathrm{C}$ NMR spectrum of $(R, R)-\mathbf{5 a}\left(\mathrm{CDCl}_{3}, 75 \mathrm{MHz}\right) \quad \mathrm{S} 18$

DEPT spectrum of $(R, R)-\mathbf{5 a}\left(\mathrm{CDCl}_{3}, 75 \mathrm{MHz}\right) \quad \mathrm{S} 19$

${ }^{1} \mathrm{H}$ NMR spectrum of $(R, R, R, R, R, R)-\mathbf{6 a}\left(\mathrm{CDCl}_{3}, 300 \mathrm{MHz}\right) \quad \mathrm{S} 20$

${ }^{13} \mathrm{C}$ NMR spectrum of $(R, R, R, R, R, R)-\mathbf{6 a}\left(\mathrm{CDCl}_{3}, 75 \mathrm{MHz}\right) \quad \mathrm{S} 21$

DEPT spectrum of $(R, R, R, R, R, R)-\mathbf{6 a}\left(\mathrm{CDCl}_{3}, 75 \mathrm{MHz}\right) \quad \mathrm{S} 22$

${ }^{1} \mathrm{H}$ NMR spectrum of $(R, R, R, R, R, R)-7 \mathbf{a}\left(\mathrm{D}_{2} \mathrm{O}, 300 \mathrm{MHz}\right) \quad \mathrm{S} 23$

${ }^{13} \mathrm{C}$ NMR spectrum of $(R, R, R, R, R, R)-7 \mathbf{a}\left(\mathrm{D}_{2} \mathrm{O}, 75 \mathrm{MHz}\right) \quad \mathrm{S} 24$

DEPT spectrum of $(R, R, R, R, R, R)-7 \mathbf{a}\left(\mathrm{D}_{2} \mathrm{O}, 75 \mathrm{MHz}\right) \quad \mathrm{S} 25$

${ }^{1} \mathrm{H}$ NMR spectrum of $(S, S, R, R, S, S)$-6a $\left(\mathrm{CDCl}_{3}, 300 \mathrm{MHz}\right) \quad \mathrm{S} 26$

$\begin{array}{ll}{ }^{13} \mathrm{C} \text { NMR spectrum of }(S, S, R, R, S, S)-\mathbf{6 a}\left(\mathrm{CDCl}_{3}, 75 \mathrm{MHz}\right) & \mathrm{S} 27\end{array}$

DEPT spectrum of $(S, S, R, R, S, S)-\mathbf{6 a}\left(\mathrm{CDCl}_{3}, 75 \mathrm{MHz}\right) \quad \mathrm{S} 28$

$\begin{array}{ll}{ }^{1} \mathrm{H} \text { NMR spectrum of }(S, S, R, R, S, S)-\mathbf{7 a}\left(\mathrm{CDCl}_{3}, 300 \mathrm{MHz}\right) & \mathrm{S} 29\end{array}$

${ }^{13} \mathrm{C}$ NMR spectrum of $(S, S, R, R, S, S)-7 \mathbf{a}\left(\mathrm{CDCl}_{3}, 75 \mathrm{MHz}\right) \quad \mathrm{S} 30$

DEPT spectrum of $(S, S, R, R, S, S)-7 \mathbf{a}\left(\mathrm{CDCl}_{3}, 75 \mathrm{MHz}\right) \quad \mathrm{S} 31$

${ }^{1} \mathrm{H}$ NMR spectrum of $(R, R)-\mathbf{3 b}\left(\mathrm{CDCl}_{3}, 300 \mathrm{MHz}\right) \quad \mathrm{S} 32$

${ }^{13} \mathrm{C}$ NMR spectrum of $(R, R)-3 \mathbf{b}\left(\mathrm{CDCl}_{3}, 75 \mathrm{MHz}\right) \quad \mathrm{S} 33$

DEPT spectrum of $(R, R)-\mathbf{3 b}\left(\mathrm{CDCl}_{3}, 75 \mathrm{MHz}\right) \quad \mathrm{S} 34$ 
${ }^{1} \mathrm{H}$ NMR spectrum of $(R, R)-\mathbf{4 b}\left(\mathrm{CDCl}_{3}, 300 \mathrm{MHz}\right) \quad \mathrm{S} 35$

${ }^{13} \mathrm{C}$ NMR spectrum of $(R, R)-\mathbf{4 b}\left(\mathrm{CDCl}_{3}, 75 \mathrm{MHz}\right) \quad \mathrm{S} 36$

DEPT spectrum of $(R, R)-\mathbf{4 b}\left(\mathrm{CDCl}_{3}, 75 \mathrm{MHz}\right) \quad \mathrm{S} 37$

${ }^{1} \mathrm{H}$ NMR spectrum of $(R, R, R, R, R, R)-5 \mathbf{b}\left(\mathrm{CDCl}_{3}, 300 \mathrm{MHz}\right) \quad \mathrm{S} 38$

${ }^{13} \mathrm{C}$ NMR spectrum of $(R, R, R, R, R, R)-\mathbf{5 b}\left(\mathrm{CDCl}_{3}, 75 \mathrm{MHz}\right) \quad \mathrm{S} 39$

DEPT spectrum of $(R, R, R, R, R, R)-\mathbf{5 b}\left(\mathrm{CDCl}_{3}, 75 \mathrm{MHz}\right) \quad \mathrm{S} 40$

${ }^{1} \mathrm{H}$ NMR spectrum of $(R, R, R, R, R, R)-\mathbf{6 b}\left(\mathrm{CDCl}_{3}, 300 \mathrm{MHz}\right) \quad \mathrm{S} 41$

${ }^{13} \mathrm{C}$ NMR spectrum of $(R, R, R, R, R, R)-\mathbf{6 b}\left(\mathrm{CDCl}_{3}, 75 \mathrm{MHz}\right) \quad \mathrm{S} 42$

DEPT spectrum of $(R, R, R, R, R, R)-\mathbf{6 b}\left(\mathrm{CDCl}_{3}, 75 \mathrm{MHz}\right) \quad \mathrm{S} 43$

${ }^{1} \mathrm{H}$ NMR spectrum of $(R, R, R, R, R, R)-7 \mathbf{b}\left(\mathrm{D}_{2} \mathrm{O}, 300 \mathrm{MHz}\right) \quad \mathrm{S} 44$

${ }^{13} \mathrm{C}$ NMR spectrum of $(R, R, R, R, R, R)-7 \mathbf{b}\left(\mathrm{D}_{2} \mathrm{O}, 75 \mathrm{MHz}\right) \quad \mathrm{S} 45$

DEPT spectrum of $(R, R, R, R, R, R)-7 \mathbf{b}\left(\mathrm{D}_{2} \mathrm{O}, 75 \mathrm{MHz}\right) \quad \mathrm{S} 46$

${ }^{1} \mathrm{H}$ NMR spectrum of $(R, R)-8\left(\mathrm{CDCl}_{3}, 300 \mathrm{MHz}\right) \quad \mathrm{S} 47$

${ }^{13} \mathrm{C}$ NMR spectrum of $(R, R)-\mathbf{8}\left(\mathrm{CDCl}_{3}, 75 \mathrm{MHz}\right) \quad \mathrm{S} 48$

$\begin{array}{lr}\text { DEPT spectrum of }(R, R)-8\left(\mathrm{CDCl}_{3}, 75 \mathrm{MHz}\right) & \mathrm{S} 49\end{array}$

${ }^{1} \mathrm{H}$ NMR spectrum of $(R, R)-9\left(\mathrm{CDCl}_{3}, 300 \mathrm{MHz}\right) \quad$ S50

${ }^{13} \mathrm{C}$ NMR spectrum of $(R, R)-9\left(\mathrm{CDCl}_{3}, 75 \mathrm{MHz}\right) \quad \mathrm{S} 51$

DEPT spectrum of $(R, R)-9\left(\mathrm{CDCl}_{3}, 75 \mathrm{MHz}\right) \quad \mathrm{S} 52$

${ }^{1} \mathrm{H}$ NMR spectrum of $(R, R)-\mathbf{1 0}\left(\mathrm{CDCl}_{3}, 300 \mathrm{MHz}\right) \quad$ S53

${ }^{13} \mathrm{C}$ NMR spectrum of $(R, R)-\mathbf{1 0}\left(\mathrm{CDCl}_{3}, 75 \mathrm{MHz}\right) \quad \mathrm{S} 54$

DEPT spectrum of $(R, R)-\mathbf{1 0}\left(\mathrm{CDCl}_{3}, 75 \mathrm{MHz}\right) \quad \mathrm{S} 55$

${ }^{1} \mathrm{H}$ NMR spectrum of $(R, R, R, R, R, R)-\mathbf{1 1}\left(\mathrm{CDCl}_{3}, 300 \mathrm{MHz}\right) \quad$ S56

${ }^{13} \mathrm{C}$ NMR spectrum of $(R, R, R, R, R, R)-11\left(\mathrm{CDCl}_{3}, 75 \mathrm{MHz}\right) \quad \mathrm{S} 57$

DEPT spectrum of $(R, R, R, R, R, R)-\mathbf{1 1}\left(\mathrm{CDCl}_{3}, 75 \mathrm{MHz}\right) \quad \mathrm{S} 58$

${ }^{1} \mathrm{H}$ NMR spectrum of $(R, R, R, R, R, R)-\mathbf{1 2}\left(\mathrm{CDCl}_{3}, 300 \mathrm{MHz}\right) \quad$ S59

${ }^{13} \mathrm{C}$ NMR spectrum of $(R, R, R, R, R, R)-\mathbf{1 2}\left(\mathrm{CDCl}_{3}, 75 \mathrm{MHz}\right) \quad \mathrm{S} 60$

DEPT spectrum of $(R, R, R, R, R, R)-\mathbf{1 2}\left(\mathrm{CDCl}_{3}, 75 \mathrm{MHz}\right) \quad$ S61

${ }^{1} \mathrm{H}$ NMR spectrum of $(R, R, R, R, R, R)-13\left(\mathrm{D}_{2} \mathrm{O}, 300 \mathrm{MHz}\right) \quad \mathrm{S} 62$

${ }^{13} \mathrm{C}$ NMR spectrum of $(R, R, R, R, R, R)-\mathbf{1 3}\left(\mathrm{D}_{2} \mathrm{O}, 75 \mathrm{MHz}\right) \quad \mathrm{S} 63$

DEPT spectrum of $(R, R, R, R, R, R)-\mathbf{1 3}\left(\mathrm{D}_{2} \mathrm{O}, 75 \mathrm{MHz}\right) \quad \mathrm{S} 64$ 


\section{General techniques}

Reagents were purchased from commercial sources and used without previous purification. Compounds $(R, R)-\mathbf{2 a}, \mathbf{b}$ and $(S, S)-\mathbf{2 a}, \mathbf{b}$ were prepared as previously described $^{15,16}$ from $(R, R)-\mathbf{1}$ or $(S, S)-\mathbf{1}$, respectively. Solvents were purified by distillation with the appropriate drying agent. Mass spectra were performed using either ESI or (EI) ionization source mass spectrometer. NMR experiments $\left({ }^{1} \mathrm{H},{ }^{13} \mathrm{C}\right.$ NMR and DEPT) were done in a spectrometer operating at $300 \mathrm{MHz}$ for proton nucleus. The AT-rich sequence (5'-GCC AAG AAA GAA AAA AGA CGC), and its complementary (3'-CGG TTC TTT CTT TTT TCT GCG) were synthesized using an automated DNA synthesizer and purified by gel electrophoresis. The GC-rich one 5'-CCT TCG CCT CGC ACA TAG CC and its complementary 3'-GGA AGC GGA GCG TGT ATC GG were purchased from commercial sources and purified by gel electrophoresis. UV melting profiles were obtained on a UV/VIS spectrophotometer by measuring the sample absorbance (in OD) in a quartz cuvette at $260 \mathrm{~nm}$. The sample compartment was equipped with a Peltier thermostatted cell holder. The concentration of duplex DNA was $2 \mu \mathrm{M}(40-42 \mu \mathrm{M}$ base pairs) in $5 \mathrm{mM}$ Tris$\mathrm{HCl}$ buffer containing $5 \mathrm{mM} \mathrm{NaCl}$ at $\mathrm{pH} 7.03$, in the absence and in the presence of 40-42 $\mu \mathrm{M}$ of the corresponding polyamine. The melting experiments were carried out using UVVisible ChemStation software by increasing the temperature from $10-90{ }^{\circ} \mathrm{C}$ in $0.5-1{ }^{\circ} \mathrm{C}$ steps and recording the absorbance at $260 \mathrm{~nm}$ after temperature stabilisation. The $T_{\mathrm{m}}$ values were obtained by polynomial fitting of the obtained melting curves and taken as the temperature corresponding to the half-dissociation of the duplexes.

Molecular modeling for the visualization of the possible interactions was performed as follows. Preliminary conformational searches of the tri-protonated polyamine compounds were carried out using a Monte Carlo approach with MMFFaq force field minimizations 
(available in Spartan 04). Cyclohexanediamine moiety retained its chair-like conformation setting the substituents in trans-diequatorial disposition. The DNA model was built as ideal B-DNA strand (Macromodel). The lowest-energy conformations of the selected polyamines were manually docked to the DNA in different relative dispositions. The system was minimized (MMFFaq) in every case by constraining the DNA strand to its initial conformation in order to avoid strand disruption. Thus, we obtained an estimation of the best aliphatic spacers for an optimal charge distribution (see text) to fit phosphate backbone of ideal DNA. From this simple analysis, we selected two different derivatives $(\mathrm{n}=1$ and 4).

\section{Synthesis of different stereoisomers of polyamines 7a,b}

Compound $(\boldsymbol{R}, \boldsymbol{R})-3 \mathrm{a}$. In a flask under nitrogen atmosphere, $1 \mathrm{mmol}$ of $(R, R)-\mathbf{2 a}$ was dissolved in $7 \mathrm{ml}$ of dry toluene in the presence of a large excess of $\mathrm{Cs}_{2} \mathrm{CO}_{3}(10 \mathrm{mmol})$. The mixture was heated to $110^{\circ} \mathrm{C}$ and after $30 \mathrm{~min}, 5 \mathrm{mmol}$ of MeI were added dropwise. The reaction was followed by TLC in $\mathrm{CH}_{2} \mathrm{Cl}_{2}$ and the total consumption of the starting material was observed after 1 hour. Evaporation of solvent to dryness and subsequent chromatographic purification using $\mathrm{CH}_{2} \mathrm{Cl}_{2}$ afforded the desired product in quantitative

yield. White solid M. P. $=177-179^{\circ} \mathrm{C} ;[\alpha]_{\mathrm{D}}{ }^{20}=-16.6\left(\mathrm{c}=0.76, \mathrm{CHCl}_{3}\right) ; \mathrm{IR}\left(\mathrm{cm}^{-1}\right)(\mathrm{KBr})$ 3058, 3030; Rf (1.1\% AcOEt/ $\left.\mathrm{CH}_{2} \mathrm{Cl}_{2}\right)$ 0.66; ${ }^{1} \mathrm{H} \mathrm{NMR}\left(\mathrm{CDCl}_{3}, 300 \mathrm{MHz}\right) \delta$ (ppm) 1.092.00 (bm, 9H), 2.00-2.27(m, 1H), $2.43(\mathrm{~s}, 6 \mathrm{H}), 2.64(\mathrm{~s}, 3 \mathrm{H}), 2.93-3.24(\mathrm{~m}, 2 \mathrm{H}), 3.30-3.60$ (m, 2H), 3.82-4.13 (m, 2H), 7.12-7.58 (m, 19H), $7.74(\mathrm{~d}, J=8.1 \mathrm{~Hz}, 2 \mathrm{H}), 7.82(\mathrm{~d}, J=8.1$ $\mathrm{Hz}, 2 \mathrm{H}) ;{ }^{13} \mathrm{C} \mathrm{NMR}\left(\mathrm{CDCl}_{3}, 75 \mathrm{MHz}\right) \delta(\mathrm{ppm}) 21.5\left(\mathrm{CH}_{3}\right), 24.9\left(\mathrm{CH}_{2}\right), 25.4\left(\mathrm{CH}_{2}\right), 28.7$ $\left(\mathrm{CH}_{2}\right), 28.9\left(\mathrm{CH}_{3}\right), 31.1\left(\mathrm{CH}_{2}\right), 32.3\left(\mathrm{CH}_{2}\right), 41.8\left(\mathrm{CH}_{2}\right), 57.0(\mathrm{CH}), 57.6(\mathrm{CH}), 61.3\left(\mathrm{CH}_{2}\right)$, $86.4(\mathrm{C}), 126.8(\mathrm{CH}), 127.3(\mathrm{CH}), 127.4(\mathrm{CH}), 127.7(\mathrm{CH}), 128.6(\mathrm{CH}), 129.5(\mathrm{CH}), 129.6$ 
(CH), 136.5 (C), 138.4 (C), 142.8 (C), 143.0 (C), 144.1 (C). HRMS/EI (m/z) calculated for $\mathrm{C}_{43} \mathrm{H}_{48} \mathrm{~N}_{2} \mathrm{~S}_{2} \mathrm{O}_{5} 736.30046$ found 736.29690 .

Compound $(\boldsymbol{S}, \boldsymbol{S})-\mathbf{3 a}$. The synthesis of the title compound was performed as for its enantiomer but starting from $(S, S)$-2a, showing the expected spectroscopic and analytical data. Yield: quantitative; $[\alpha]_{\mathrm{D}}^{20}=+15.6\left(\mathrm{c}=0.76, \mathrm{CHCl}_{3}\right)$.

Compound $(\boldsymbol{R}, \boldsymbol{R})-\mathbf{3 b}$. The synthesis of the title compound was performed as for $(R, R)-\mathbf{3 a}$ but starting from $(R, R)-\mathbf{2 b}$. Yield: quantitative; white solid M. P. $=69-72{ }^{\circ} \mathrm{C} ;[\alpha]_{\mathrm{D}}{ }^{20}=-8.5$ $\left(\mathrm{c}=0.61, \mathrm{CHCl}_{3}\right) ; \mathrm{IR}\left(\mathrm{cm}^{-1}\right)(\mathrm{KBr}) 3058,3030 ; \mathrm{Rf}\left(1.1 \% \mathrm{AcOEt} / \mathrm{CH}_{2} \mathrm{Cl}_{2}\right) 0.66 ;{ }^{1} \mathrm{H} \mathrm{NMR}$ $\left(\mathrm{CDCl}_{3}, 300 \mathrm{MHz}\right) \delta(\mathrm{ppm}) 1.10-1.90(\mathrm{~m}, 16 \mathrm{H}), 2.40(\mathrm{~s}, 6 \mathrm{H}), 2.60(\mathrm{~s}, 3 \mathrm{H}), 3.10-3.20(\mathrm{~m}$, 2H), 3.20-3.40 (m, 2H), 3.80-4.00 (m, 2H), 7.10-7.40 (m, $15 \mathrm{H}), 7.50(\mathrm{~d}, J=8.3 \mathrm{~Hz}, 4 \mathrm{H})$, $7.77(\mathrm{~d}, J=8.1 \mathrm{~Hz}, 2 \mathrm{H}), 7.81(\mathrm{~d}, J=8.1 \mathrm{~Hz}, 2 \mathrm{H}) ;{ }^{13} \mathrm{C} \mathrm{NMR}\left(\mathrm{CDCl}_{3}, 75 \mathrm{MHz}\right) \delta(\mathrm{ppm})$ $21.2\left(\mathrm{CH}_{3}\right), 24.7\left(\mathrm{CH}_{2}\right), 25.2\left(\mathrm{CH}_{2}\right), 25.7\left(\mathrm{CH}_{2}\right), 27.1\left(\mathrm{CH}_{2}\right), 28.6\left(\mathrm{CH}_{2}\right), 28.6\left(\mathrm{CH}_{3}\right), 29.7$ $\left(\mathrm{CH}_{2}\right), 30.4\left(\mathrm{CH}_{2}\right), 32.4\left(\mathrm{CH}_{2}\right), 44.1\left(\mathrm{CH}_{2}\right), 56.8(\mathrm{CH}), 57.4(\mathrm{CH}), 63.2\left(\mathrm{CH}_{2}\right), 86.0(\mathrm{C})$, $126.5(\mathrm{CH}), 127.0(\mathrm{CH})$, 127.1(CH), $127.4(\mathrm{CH}), 128.4(\mathrm{CH}), 129.3(\mathrm{CH}), 129.4(\mathrm{CH})$, 136.2 (C), 138.6 (C),138.6 (C), 142.6 (C), 143.0 (C), 144.2 (C); ESI-MS (m/z) 801.5 $\left[(\mathrm{M}+\mathrm{Na})^{+}, 100\right]$. Elemental analysis: calculated for $\mathrm{C}_{46} \mathrm{H}_{54} \mathrm{~N}_{2} \mathrm{O}_{5} \mathrm{~S}_{2} \mathrm{C}, 70.92 ; \mathrm{H}, 6.99 ; \mathrm{N}$, 3.60 found $\mathrm{C}, 70.75 ; \mathrm{H}, 7.20 ; \mathrm{N}, 3.40$.

Compound $(\boldsymbol{S}, \boldsymbol{S})$-3b. The synthesis of the title compound was performed as for $(R, R)-\mathbf{3 a}$ but starting from $(S, S)-\mathbf{2} \mathbf{b}$, showing the expected spectroscopic and analytical data. Yield: quantitative; $[\alpha]_{\mathrm{D}}^{20}=+8.3\left(\mathrm{c}=0.68, \mathrm{CHCl}_{3}\right)$.

Alcohol $(\boldsymbol{R}, \boldsymbol{R})$-4a. Trityl group deprotection was carried out by dissolving $1 \mathrm{mmol}$ of $(R, R)-3 \mathbf{a}$ in $12 \mathrm{ml}$ of $\mathrm{CH}_{2} \mathrm{Cl}_{2}$ and $4.6 \mathrm{ml}$ of $\mathrm{MeOH}$. Then, $3 \mathrm{mmol}$ of TFA were added at room temperature. After $12 \mathrm{~h}$, the reaction was complete by TLC $\left(\mathrm{CH}_{2} \mathrm{Cl}_{2}\right.$ : AcOEt 9:0.5), 
and the solvent was evaporated to dryness. The title product was quantitatively obtained after filtration over silica gel using the same mixture of solvents than for the TLC. Yield: 90\%; white solid; M. P. $=72-74{ }^{\circ} \mathrm{C} ;[\alpha]_{\mathrm{D}}{ }^{20}=-1.6\left(\mathrm{c}=0.61, \mathrm{CHCl}_{3}\right) ; \mathrm{IR}\left(\mathrm{cm}^{-1}\right)(\mathrm{KBr}) 3546$, 2937, 2864; Rf (5.8\% AcOEt/ $\left.\mathrm{CH}_{2} \mathrm{Cl}_{2}\right)$ 0.10; ${ }^{1} \mathrm{H} \mathrm{NMR}\left(\mathrm{CDCl}_{3}, 300 \mathrm{MHz}\right) \delta(\mathrm{ppm}) 1.03-$ 1.94 (m, 9H), 2.0-2.17 (m, 2H), 2.44 (s, 6H), 2.57 (s, 3H), 3.25-3.64 (m, 3H), 3.71-4.01 (m, $3 \mathrm{H}), 7.19-7.38(\mathrm{~m}, 4 \mathrm{H}), 7.69(\mathrm{~d}, J=8.1 \mathrm{~Hz}, 2 \mathrm{H}), 7.77(\mathrm{~d}, J=8.1 \mathrm{~Hz}, 2 \mathrm{H}) ;{ }^{13} \mathrm{C}$ NMR $\left(\mathrm{CDCl}_{3}, 75 \mathrm{MHz}\right) \delta(\mathrm{ppm}) 21.5\left(\mathrm{CH}_{3}\right), 24.7\left(\mathrm{CH}_{2}\right), 25.2\left(\mathrm{CH}_{2}\right), 28.3\left(\mathrm{CH}_{2}\right), 28.6\left(\mathrm{CH}_{3}\right)$, 31.0 $\left(\mathrm{CH}_{2}\right), 33.7\left(\mathrm{CH}_{2}\right), 40.7\left(\mathrm{CH}_{2}\right), 57.4(\mathrm{CH}), 58.3(\mathrm{CH}), 60.1\left(\mathrm{CH}_{2}\right), 127.2(\mathrm{CH}), 127.3$ (CH), $129.6(\mathrm{CH}), 129.7(\mathrm{CH}), 136.3(\mathrm{C}), 137.9$ (C), $143.2(\mathrm{C}), 143.3(\mathrm{C})$; EI-MS (m/z) 339.1[(M-Ts $)^{+}$, 100]. Elemental analysis: calculated for $\mathrm{C}_{24} \mathrm{H}_{34} \mathrm{~N}_{2} \mathrm{O}_{5} \mathrm{~S}_{2} \mathrm{C}, 58.27 ; \mathrm{H}, 6.93$; N, 5.66 found C, 58.02; H, 6.99; N, 5.34.

Alcohol $(\boldsymbol{S}, \boldsymbol{S})$-4a. The synthesis of the title compound was performed as for its enantiomer but starting from $(S, S)$-3a, showing the expected spectroscopic and analytical data. Yield: $92 \% ;[\alpha]_{\mathrm{D}}^{20}=+1.6\left(\mathrm{c}=0.74, \mathrm{CHCl}_{3}\right)$.

Alcohol $(\boldsymbol{R}, \boldsymbol{R})-\mathbf{4 b}$. The synthesis of the title compound was performed as for $(R, R)-\mathbf{4 a}$ but starting from $(R, R)-3 \mathbf{b}$. Yield: $90 \%$; white solid; M. P. $=43-45^{\circ} \mathrm{C} ;[\alpha]_{\mathrm{D}}{ }^{20}=-17.6(\mathrm{c}=0.63$, $\left.\mathrm{CHCl}_{3}\right)$; IR $\left(\mathrm{cm}^{-1}\right)(\mathrm{KBr}) 3546,2937,2864 ; \mathrm{Rf}\left(5.8 \% \mathrm{AcOEt} / \mathrm{CH}_{2} \mathrm{Cl}_{2}\right)$ 0.10; ${ }^{1} \mathrm{H} \mathrm{NMR}$ $\left(\mathrm{CDCl}_{3}, 300 \mathrm{MHz}\right) \delta(\mathrm{ppm}) 1.10-1.90(\mathrm{~m}, 16 \mathrm{H}), 2.40(\mathrm{~s}, 6 \mathrm{H}), 2.60(\mathrm{~s}, 3 \mathrm{H}), 3.10-3.30(\mathrm{~m}$, 2H), 3.60-4.00 (m, 4H), $7.28(\mathrm{~d}, J=5.2 \mathrm{~Hz}, 2 \mathrm{H}), 7.31(\mathrm{~d}, J=6.6 \mathrm{~Hz}, 2 \mathrm{H}), 7.71(\mathrm{~d}, J=8.3$ $\mathrm{Hz}, 2 \mathrm{H},), 7.77(\mathrm{~d}, J=8.3 \mathrm{~Hz}, 2 \mathrm{H}) ;{ }^{13} \mathrm{C} \mathrm{NMR}\left(\mathrm{CDCl}_{3}, 75 \mathrm{MHz}\right) \delta(\mathrm{ppm}) 21.4\left(\mathrm{CH}_{3}\right), 24.8$ $\left(\mathrm{CH}_{2}\right), 25.1\left(\mathrm{CH}_{2}\right), 25.3\left(\mathrm{CH}_{2}\right), 27.0\left(\mathrm{CH}_{2}\right), 28.7\left(\mathrm{CH}_{2}\right), 30.4\left(\mathrm{CH}_{2}\right), 32.4\left(\mathrm{CH}_{2}\right), 40.2$ $\left(\mathrm{CH}_{2}\right), 57.1(\mathrm{CH}), 57.6(\mathrm{CH}), 62.6\left(\mathrm{CH}_{2}\right), 127.2(\mathrm{CH}), 127.2(\mathrm{CH}), 129.4(\mathrm{CH}), 129.5$ $(\mathrm{CH}), 136.6(\mathrm{C}), 138.6(\mathrm{C}), 142.8(\mathrm{C}), 143.2(\mathrm{C}) ; \mathrm{ESI}-\mathrm{MS}(\mathrm{m} / \mathrm{z}) 559.4\left[(\mathrm{M}+\mathrm{Na})^{+}, 100\right]$. 
Elemental analysis: calculated for $\mathrm{C}_{27} \mathrm{H}_{40} \mathrm{~N}_{2} \mathrm{O}_{5} \mathrm{~S}_{2} \mathrm{C}, 60.42 ; \mathrm{H}, 7.51 ; \mathrm{N}, 5.22$ found $\mathrm{C}, 60.20$; H, 7.60; N, 5.05.

Alcohol $(\boldsymbol{S}, \boldsymbol{S})-\mathbf{4 b}$. The synthesis of the title compound was performed as for $(R, R)-\mathbf{4 a}$ but starting from $(S, S)-\mathbf{3 b}$, showing the expected spectroscopic and analytical data. Yield: $88 \%$; $[\alpha]_{\mathrm{D}}^{20}=+17.6\left(\mathrm{c}=0.63, \mathrm{CHCl}_{3}\right)$.

Mesylate $(\boldsymbol{R}, \boldsymbol{R})-5 \mathrm{a}$. To a solution of $(R, R)-4 \mathbf{a}(1 \mathrm{mmol})$ in $10 \mathrm{ml}$ of $\mathrm{CH}_{2} \mathrm{Cl}_{2}$ and $0.3 \mathrm{ml}$ of $\mathrm{NEt}_{3}$ under nitrogen atmosphere, methanosulfonyl chloride $(1.5 \mathrm{mmol}, 0.17 \mathrm{ml})$ was added at $0{ }^{\circ} \mathrm{C}$. The mixture was stirred at RT until consumption of the starting material. Then, the reaction mixture was extracted with $\mathrm{CH}_{2} \mathrm{Cl}_{2}$ and $1 \mathrm{~N} \mathrm{HCl}$, the combined organic layers were dried and evaporated yielding the title compound, which was used for the next step without further purification. Yield $=$ quantitative; white solid M. P. $=84-86^{\circ} \mathrm{C} ;[\alpha]_{\mathrm{D}}{ }^{20}=-6.3(\mathrm{c}=$ 0.53, $\left.\mathrm{CHCl}_{3}\right) ; \mathrm{IR}\left(\mathrm{cm}^{-1}\right)(\mathrm{KBr}) 2939,2865 ; \mathrm{Rf}\left(1.2 \% \mathrm{AcOEt} / \mathrm{CH}_{2} \mathrm{Cl}_{2}\right)$ 0.5; ${ }^{1} \mathrm{H} \mathrm{NMR}\left(\mathrm{CDCl}_{3}\right.$, $300 \mathrm{MHz}) \delta(\mathrm{ppm})$ 0.92-1.75 (bm, 8H), 2.14-2.39 (m, 2H), $2.40(\mathrm{~s}, 6 \mathrm{H}), 2.54(\mathrm{~s}, 3 \mathrm{H}), 3.03$ (s, 3H), 3.21-3.51 (m, 2H), 3.64-3.98 (m, 2H), 4.18-4.47 (m, 2H), $7.28(\mathrm{~d}, J=7.99 \mathrm{~Hz}$, 4H), $7.66(\mathrm{~d}, J=7.99 \mathrm{~Hz}, 2 \mathrm{H}), 7.74(\mathrm{~d}, J=7.99 \mathrm{~Hz}, 2 \mathrm{H}) ;{ }^{13} \mathrm{C} \mathrm{NMR}\left(\mathrm{CDCl}_{3}, 75 \mathrm{MHz}\right) \delta$ (ppm) $21.09\left(\mathrm{CH}_{3}\right), 24.35\left(\mathrm{CH}_{2}\right), 24.87\left(\mathrm{CH}_{2}\right), 28.02\left(\mathrm{CH}_{2}\right), 28.36\left(\mathrm{CH}_{3}\right), 29.95\left(\mathrm{CH}_{2}\right)$, $30.96\left(\mathrm{CH}_{2}\right), 36.82\left(\mathrm{CH}_{3}\right), 40.49\left(\mathrm{CH}_{2}\right), 57.13(\mathrm{CH}), 57.92(\mathrm{CH}), \quad 68.57\left(\mathrm{CH}_{2}\right), 126.79$ (CH), $126.89(\mathrm{CH}), 129.38(\mathrm{CH}), 136.03(\mathrm{C}), 137.42$ (C),142.99 (C), 143.02 (C); EI-MS $(\mathrm{m} / \mathrm{z}) 417.0\left[(\mathrm{M}-\mathrm{Ts})^{+}, 100\right]$. Elemental analysis: calculated for $\mathrm{C}_{25} \mathrm{H}_{36} \mathrm{~N}_{2} \mathrm{O}_{7} \mathrm{~S}_{3} \mathrm{C}, 52.42 ; \mathrm{H}$, 6.34; N, 4.89 found $\mathrm{C}, 52.22 ; \mathrm{H}, 6.54 ; \mathrm{N}, 4.57$.

Mesylate $(\boldsymbol{S}, \boldsymbol{S})$-5a. The synthesis of the title compound was performed as for its enantiomer but starting from $(S, S)$-4a, showing the expected spectroscopic and analytical data. Yield: quantitative; $[\alpha]_{\mathrm{D}}^{20}=+6.4\left(\mathrm{c}=0.49, \mathrm{CHCl}_{3}\right)$. 
Mesylate $(\boldsymbol{R}, \boldsymbol{R})-\mathbf{5 b}$. The synthesis of the title compound was performed as for $(R, R)-\mathbf{5 a}$ but starting from $(R, R)-\mathbf{4 b}$. Yield: quantitative; white foamy solid M. P. $=42-48^{\circ} \mathrm{C} ;[\alpha]_{\mathrm{D}}{ }^{20}=$ $-13.9\left(\mathrm{c}=0.54, \mathrm{CHCl}_{3}\right) ; \mathrm{IR}\left(\mathrm{cm}^{-1}\right)(\mathrm{KBr}) 2939,2865 ; \mathrm{Rf}\left(1.2 \% \mathrm{AcOEt} / \mathrm{CH}_{2} \mathrm{Cl}_{2}\right) 0.5 ;{ }^{1} \mathrm{H}$ NMR $\left(\mathrm{CDCl}_{3}, 300 \mathrm{MHz}\right) \delta(\mathrm{ppm})\left(\mathrm{CDCl}_{3}, 300 \mathrm{MHz}\right) \delta(\mathrm{ppm}) 1.10-1.80(\mathrm{~m}, 16 \mathrm{H}), 2.30(\mathrm{~s}$, $6 \mathrm{H}), 2.50(\mathrm{~s}, 3 \mathrm{H}), 2.90(\mathrm{~s}, 3 \mathrm{H}), 3.10-3.30(\mathrm{~m}, 4 \mathrm{H}), 4.10-4.30(\mathrm{~m}, 2 \mathrm{H}), 7.20(\mathrm{dd}, J=8.0 \mathrm{~Hz}$, $J=3.5 \mathrm{~Hz}, 4 \mathrm{H}), 7.71(\mathrm{~d}, J=8.3 \mathrm{~Hz}, 2 \mathrm{H}), 7.77(\mathrm{~d}, J=8.2 \mathrm{~Hz}, 2 \mathrm{H}) ;{ }^{13} \mathrm{C} \mathrm{NMR}\left(\mathrm{CDCl}_{3}, 75\right.$ MHz) $\delta(\mathrm{ppm}) 21.1\left(\mathrm{CH}_{3}\right), 24.5\left(\mathrm{CH}_{2}\right), 24.5\left(\mathrm{CH}_{2}\right), 24.9\left(\mathrm{CH}_{2}\right), 26.3\left(\mathrm{CH}_{2}\right), 28.2\left(\mathrm{CH}_{2}\right)$, $28.4\left(\mathrm{CH}_{2}\right), 28.4\left(\mathrm{CH}_{3}\right), 30.0\left(\mathrm{CH}_{2}\right), 31.9\left(\mathrm{CH}_{2}\right), 36.8\left(\mathrm{CH}_{3}\right), 43.8\left(\mathrm{CH}_{2}\right), 56.9\left(\mathrm{CH}_{2}\right), 57.4$ (CH), $59.9(\mathrm{CH}), 69.9\left(\mathrm{CH}_{2}\right), 126.9(\mathrm{CH}), 129.2(\mathrm{CH}), 129.3(\mathrm{CH}), 136.1(\mathrm{C}), 138.3(\mathrm{C})$, 142.7 (C), 142.9 (C); ESI-MS (m/z) $637.4\left[(\mathrm{M}+\mathrm{Na})^{+}, 100\right]$. Elemental analysis: calculated for $\mathrm{C}_{28} \mathrm{H}_{42} \mathrm{~N}_{2} \mathrm{O}_{7} \mathrm{~S}_{3} \mathrm{C}, 54.70 ; \mathrm{H}, 6.89 ; \mathrm{N}, 4.56$ found $\mathrm{C}, 54.53 ; \mathrm{H}, 6.93 ; \mathrm{N}, 4.42$.

Mesylate $(\boldsymbol{S}, \boldsymbol{S})$-5b. The synthesis of the title compound was performed as for its enantiomer but starting from $(S, S) \mathbf{- 4 b}$, showing the expected spectroscopic and analytical data. Yield: quantitative; $[\alpha]_{\mathrm{D}}{ }^{20}=+13.8\left(\mathrm{c}=0.60, \mathrm{CHCl}_{3}\right)$.

\section{Synthesis of polyamine $(R, R, R, R, R)-13$}

Compound $(\boldsymbol{R}, \boldsymbol{R})-8$. Compound $(R, R)-\mathbf{6 b}(1.1 \mathrm{mmol})$ was dissolved in $11 \mathrm{ml}$ of dry toluene and an excess of $\mathrm{Cs}_{2} \mathrm{CO}_{3}(1.78 \mathrm{~g}, 5.45 \mathrm{mmol})$ and tetrabutylammonium bromide $(0.11 \mathrm{mmol}, 0.4 \mathrm{mg})$ were added. After refluxing the reaction mixture for half an hour, $N$ (3-bromopropyl)phthalimide (1.46 g, $5.45 \mathrm{mmol}$ ) was added and the stirring and heating continued afte consumption of the starting materials (TLC using $\mathrm{CH}_{2} \mathrm{Cl}_{2}$ as eluent). Then, the crude reaction is evaporated and extracted with $3 \mathrm{~N} \mathrm{HCl}$ and $\mathrm{CH}_{2} \mathrm{Cl}_{2}$, the combined organic layers were dried and concentrated in vacuum. The title compound was finally isolated after flash chromatographic purification using $\mathrm{CH}_{2} \mathrm{Cl}_{2}$. Yield: $75 \%$; white foamy 
solid; M. P. $71-74{ }^{\circ} \mathrm{C} ;[\alpha]_{\mathrm{D}}{ }^{20}=-24.1\left(\mathrm{c}=0.63, \mathrm{CHCl}_{3}\right) ; \mathrm{Rf}\left(\mathrm{CH}_{2} \mathrm{Cl}_{2}\right) 0.1 ; \mathrm{IR}\left(\mathrm{cm}^{-1}\right)(\mathrm{KBr})$ 1710, 2926; ${ }^{1} \mathrm{H}$ NMR $\left(\mathrm{CDCl}_{3}, 300 \mathrm{MHz}\right) \delta(\mathrm{ppm})$ 0.72-1.88 (m, 18H), 1.88-2.18 (m, 2H ), 2.18-2.54 (m, 6H), 2.68-4.05 (m, 10H), 6.93-7.52 (m, 19H), 7.52-8.09 (m, 6H) ${ }^{13} \mathrm{C}$ NMR $\left(\mathrm{CDCl}_{3}, 75 \mathrm{MHz}\right) \delta(\mathrm{ppm}) 20.9\left(\mathrm{CH}_{3}\right), 24.8\left(\mathrm{CH}_{2}\right), 25.3\left(\mathrm{CH}_{2}\right), 26.8\left(\mathrm{CH}_{2}\right), 28.8\left(\mathrm{CH}_{2}\right)$, $29.4\left(\mathrm{CH}_{2}\right), 31.4\left(\mathrm{CH}_{2}\right), 31.5\left(\mathrm{CH}_{2}\right), 35.5\left(\mathrm{CH}_{2}\right), 41.6\left(\mathrm{CH}_{2}\right), 43.7\left(\mathrm{CH}_{2}\right), 57.8(\mathrm{CH}), 58.1$ $(\mathrm{CH}), 59.8\left(\mathrm{CH}_{2}\right), 62.9\left(\mathrm{CH}_{2}\right), 62.3\left(\mathrm{CH}_{2}\right), 85.7(\mathrm{C}), 122.5(\mathrm{CH}), 126.2(\mathrm{CH}), 126.7(\mathrm{CH})$, $126.9(\mathrm{CH}), 127.1(\mathrm{CH}), 128.1(\mathrm{CH}), 128.9(\mathrm{CH}), 129.0(\mathrm{CH}), 131.6(\mathrm{CH}), 133.2(\mathrm{CH})$, 137.5 (C), 138.4 (C), 142.3 (C), 142.6 (C), 143.9 (C), 167.9 (C); ESI-MS (m/z) 952.51 $\left[(\mathrm{M}+1)^{+}, 20\right]$. Elemental analysis: calculated for $\mathrm{C}_{56} \mathrm{H}_{63} \mathrm{~N}_{3} \mathrm{O}_{7} \mathrm{~S}_{2} \mathrm{C}, 70.48 ; \mathrm{H}, 6.65 ; \mathrm{N}, 4.40$ found $\mathrm{C}, 70.27 ; \mathrm{H}, 6.85 ; \mathrm{N}, 4.10$.

Alcohol $(\boldsymbol{R}, \boldsymbol{R})$-9. The synthesis of the title compound was performed as for $(R, R)-\mathbf{4 a}$ but starting from $(R, R)-8$. Yield: $90 \%$; white foamy solid; M. P. $71-74{ }^{\circ} \mathrm{C} ;[\alpha]_{\mathrm{D}}{ }^{20}=-40.7(\mathrm{c}=$ 0.50, AcOEt); Rf (AcOEt) 0.1; IR $\left(\mathrm{cm}^{-1}\right)(\mathrm{KBr}) 1710,3417 ;{ }^{1} \mathrm{H} \mathrm{NMR}\left(\mathrm{CDCl}_{3}, 300 \mathrm{MHz}\right) \delta$ (ppm) 1.07-1.54 (m, 16H), $2.03(\mathrm{~m}, 2 \mathrm{H}), 2.24(\mathrm{~s}, 3 \mathrm{H}), 2.30(\mathrm{~s}, 3 \mathrm{H}), 2.82-3.79(\mathrm{~m}, 10 \mathrm{H})$, 2.88-3.19 (m, 1H), $7.10(\mathrm{~d}, J=6.6 \mathrm{~Hz}, 2 \mathrm{H}), 7.16(\mathrm{~d}, J=6.4 \mathrm{~Hz} 2 \mathrm{H}), 7.57-7.72(\mathrm{~m}, 8 \mathrm{H}) ;{ }^{13} \mathrm{C}$ $\operatorname{NMR}\left(\mathrm{CDCl}_{3}, 75 \mathrm{MHz}\right) \delta(\mathrm{ppm}) 21.1\left(\mathrm{CH}_{3}\right), 24.9\left(\mathrm{CH}_{2}\right), 24.9\left(\mathrm{CH}_{2}\right), 26.8\left(\mathrm{CH}_{2}\right), 29.0$ $\left(\mathrm{CH}_{2}\right), 29.6\left(\mathrm{CH}_{2}\right), 31.3\left(\mathrm{CH}_{2}\right), 32.1\left(\mathrm{CH}_{2}\right), 35.7\left(\mathrm{CH}_{2}\right), 41.7\left(\mathrm{CH}_{2}\right), 44.0\left(\mathrm{CH}_{2}\right), 58.2(\mathrm{CH})$, $58.5(\mathrm{CH}), 62.1\left(\mathrm{CH}_{2}\right), 122.7(\mathrm{CH}), 126.9(\mathrm{CH}), 127.0(\mathrm{CH}), 129.2(\mathrm{CH}), 129.3(\mathrm{CH})$, $133.6(\mathrm{CH}), 137.5(\mathrm{C}), 138.4(\mathrm{C}), 142.6$ (C), 142.9 (C), 168.0 (C); ESI-MS (m/z) 731.1 $\left.\left[(\mathrm{M}+\mathrm{Na})^{+}, 100\right], 732.1[(\mathrm{M}+1)+\mathrm{Na})^{+}, 100\right]$. Elemental analysis: calculated for $\mathrm{C}_{37} \mathrm{H}_{49} \mathrm{~N}_{3} \mathrm{O}_{7} \mathrm{~S}_{2} \mathrm{C}, 62.42 ; \mathrm{H}, 6.94 ; \mathrm{N}, 5.90$ found $\mathrm{C}, 62.23 ; \mathrm{H}, 7.05 ; \mathrm{N}, 5.58$.

Mesylate $(\boldsymbol{R}, \boldsymbol{R})-\mathbf{1 0}$. The synthesis of the title compound was performed as for $(R, R)-\mathbf{5 a}$ but starting from $(R, R)-9$. Quantitative yield, white foamy solid; M. P. $65-68{ }^{\circ} \mathrm{C} ;[\alpha]_{\mathrm{D}}{ }^{20}=$ 
$-36.4(\mathrm{c}=0.70, \mathrm{AcOEt}) ; \mathrm{Rf}\left(\mathrm{CH}_{2} \mathrm{Cl}_{2}: \mathrm{AcOEt} 95: 5\right) 0.6 ; \mathrm{IR}\left(\mathrm{cm}^{-1}\right)(\mathrm{KBr}) 1710 ;{ }^{1} \mathrm{H} \mathrm{NMR}$ $\left(\mathrm{CDCl}_{3}, 300 \mathrm{MHz}\right) \delta(\mathrm{ppm})$ 1.15-2.06 (m, 18H), $2.31(\mathrm{~s}, 3 \mathrm{H}), 2.38(\mathrm{~s}, 3 \mathrm{H}), 3.17-4.15(\mathrm{~m}$, $13 \mathrm{H}), 7.15(\mathrm{~d}, J=7.1 \mathrm{~Hz}, 2 \mathrm{H}), 7.23(\mathrm{~d}, J=6.8 \mathrm{~Hz}, 2 \mathrm{H}), 7.61-7.78(\mathrm{~m}, 8 \mathrm{H}) ;{ }^{13} \mathrm{C}$ NMR $\left(\mathrm{CDCl}_{3}, 75 \mathrm{MHz}\right) \delta(\mathrm{ppm}) 21.0\left(\mathrm{CH}_{3}\right), 24.4\left(\mathrm{CH}_{2}\right), 24.8\left(\mathrm{CH}_{2}\right), 26.2\left(\mathrm{CH}_{2}\right), 28.3\left(\mathrm{CH}_{2}\right)$, $28.8\left(\mathrm{CH}_{2}\right), 29.3\left(\mathrm{CH}_{2}\right), 31.0\left(\mathrm{CH}_{2}\right), 35.5\left(\mathrm{CH}_{2}\right), 36.7\left(\mathrm{CH}_{3}\right), 41.6\left(\mathrm{CH}_{2}\right), 43.7\left(\mathrm{CH}_{2}\right), 58.2$ $(\mathrm{CH}), 58.5(\mathrm{CH}), 69.9\left(\mathrm{CH}_{2}\right), 122.6(\mathrm{CH}), 126.7(\mathrm{CH}), 127.9(\mathrm{CH}), 129.2(\mathrm{CH}), 133.5(\mathrm{CH}$ ), $137.4(\mathrm{C}), 138.1(\mathrm{C}), 142.7$ (C), $142.8(\mathrm{C}), 167.1(\mathrm{C})$; ESI-MS (m/z) $810.1[(\mathrm{M}+1)+\mathrm{Na})^{+}$, 100]. Elemental analysis: calculated for $\mathrm{C}_{38} \mathrm{H}_{51} \mathrm{~N}_{3} \mathrm{O}_{9} \mathrm{~S}_{3} \mathrm{C}, 57.77 ; \mathrm{H}, 6.51 ; \mathrm{N}, 5.32$ found $\mathrm{C}$, $57.52 ; \mathrm{H}, 6.73 ; \mathrm{N}, 5.08$. 
${ }^{1} \mathrm{H}$ NMR spectrum of $(R, R)-\mathbf{3 a}\left(\mathrm{CDCl}_{3}, 300 \mathrm{MHz}\right)$

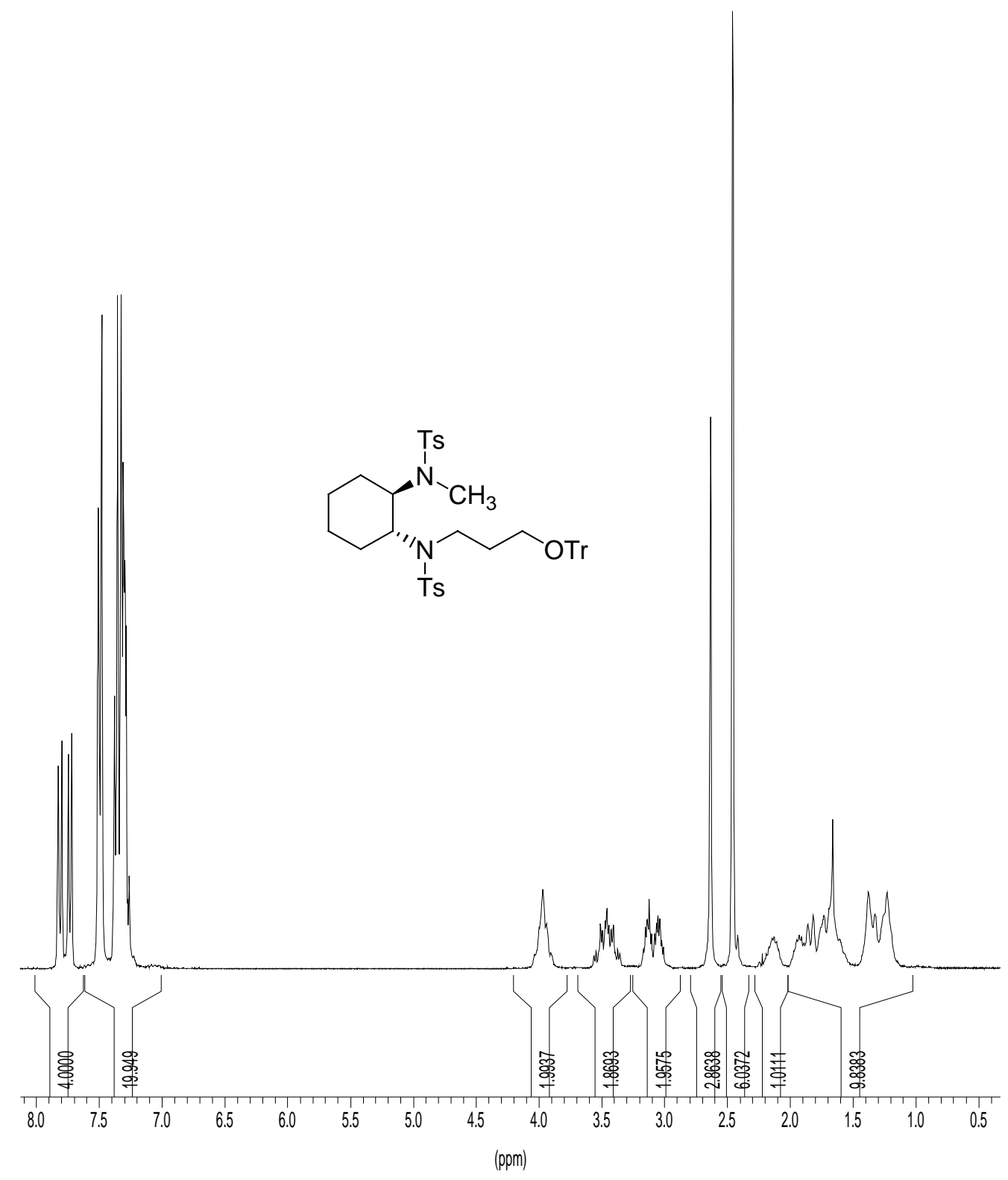


${ }^{13} \mathrm{C}$ NMR spectrum of $(R, R)-3 \mathbf{a}\left(\mathrm{CDCl}_{3}, 75 \mathrm{MHz}\right)$

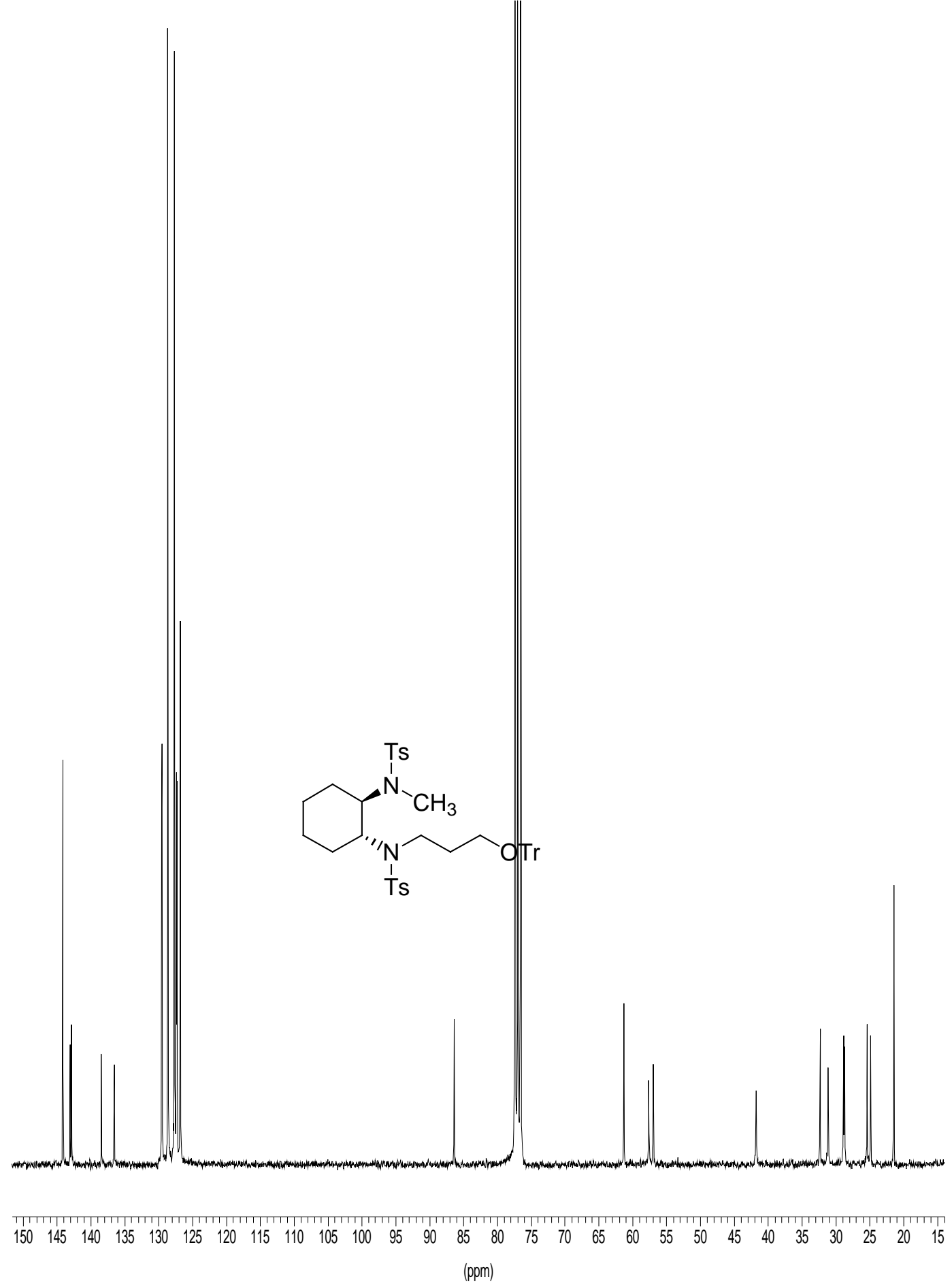


DEPT spectrum of $(R, R)-\mathbf{3 a}\left(\mathrm{CDCl}_{3}, 75 \mathrm{MHz}\right)$

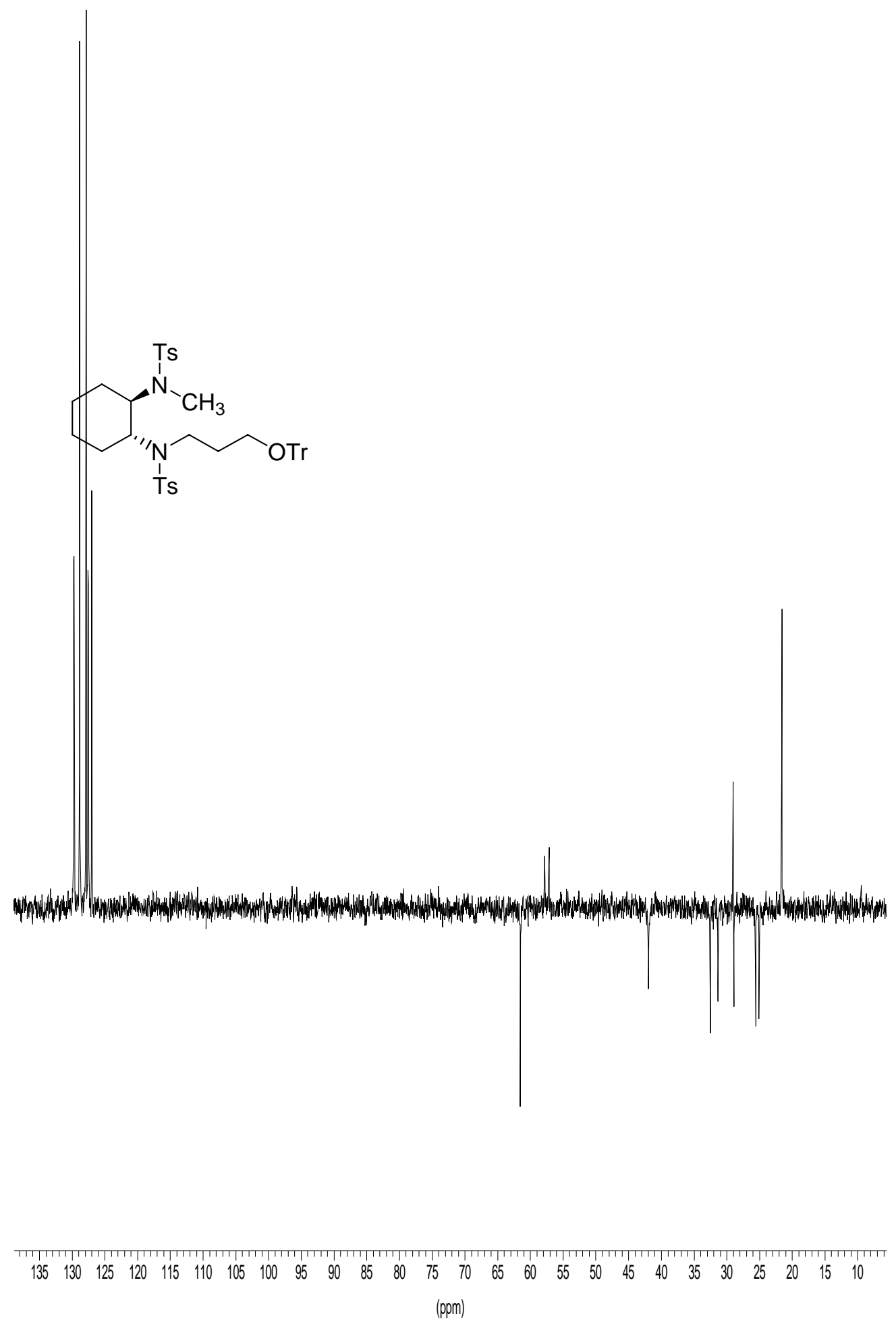


${ }^{1} \mathrm{H}$ NMR spectrum of $(R, R)-4 a\left(\mathrm{CDCl}_{3}, 300 \mathrm{MHz}\right)$

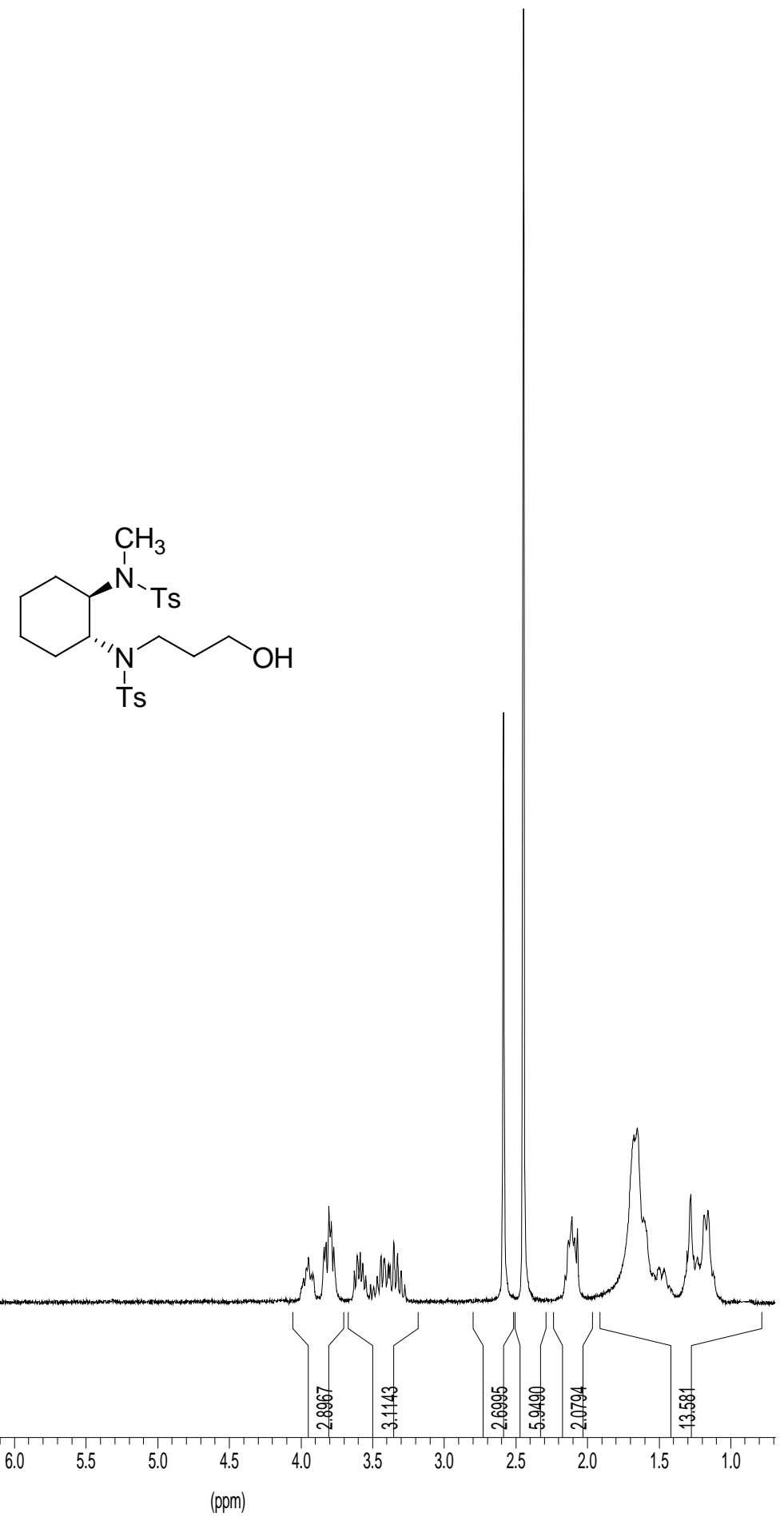


${ }^{13} \mathrm{C}$ NMR spectrum of $(R, R)-\mathbf{4 a}\left(\mathrm{CDCl}_{3}, 75 \mathrm{MHz}\right)$
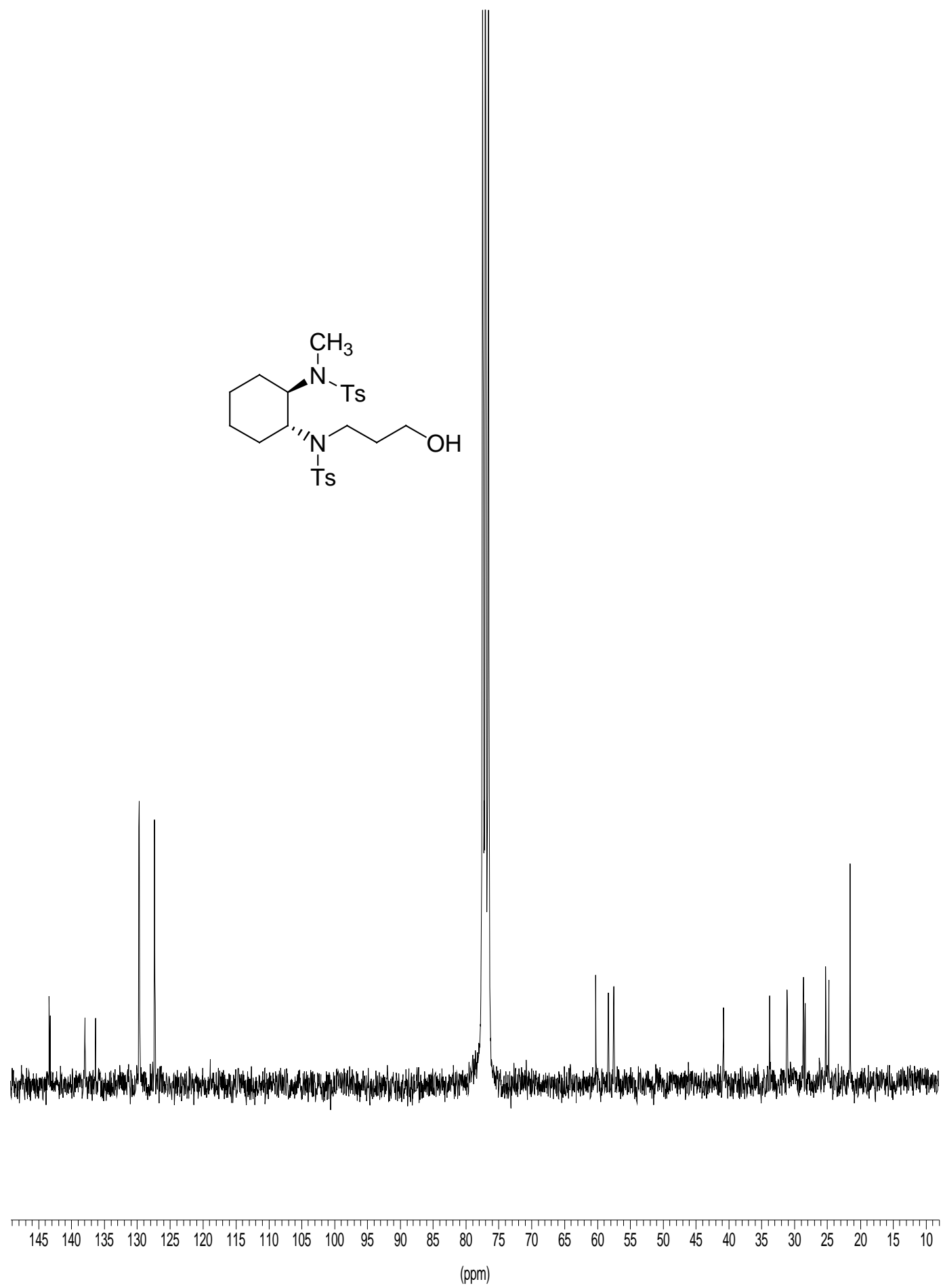

S-15 
DEPT NMR spectrum of $(R, R)-\mathbf{4 a}\left(\mathrm{CDCl}_{3}, 75 \mathrm{MHz}\right)$

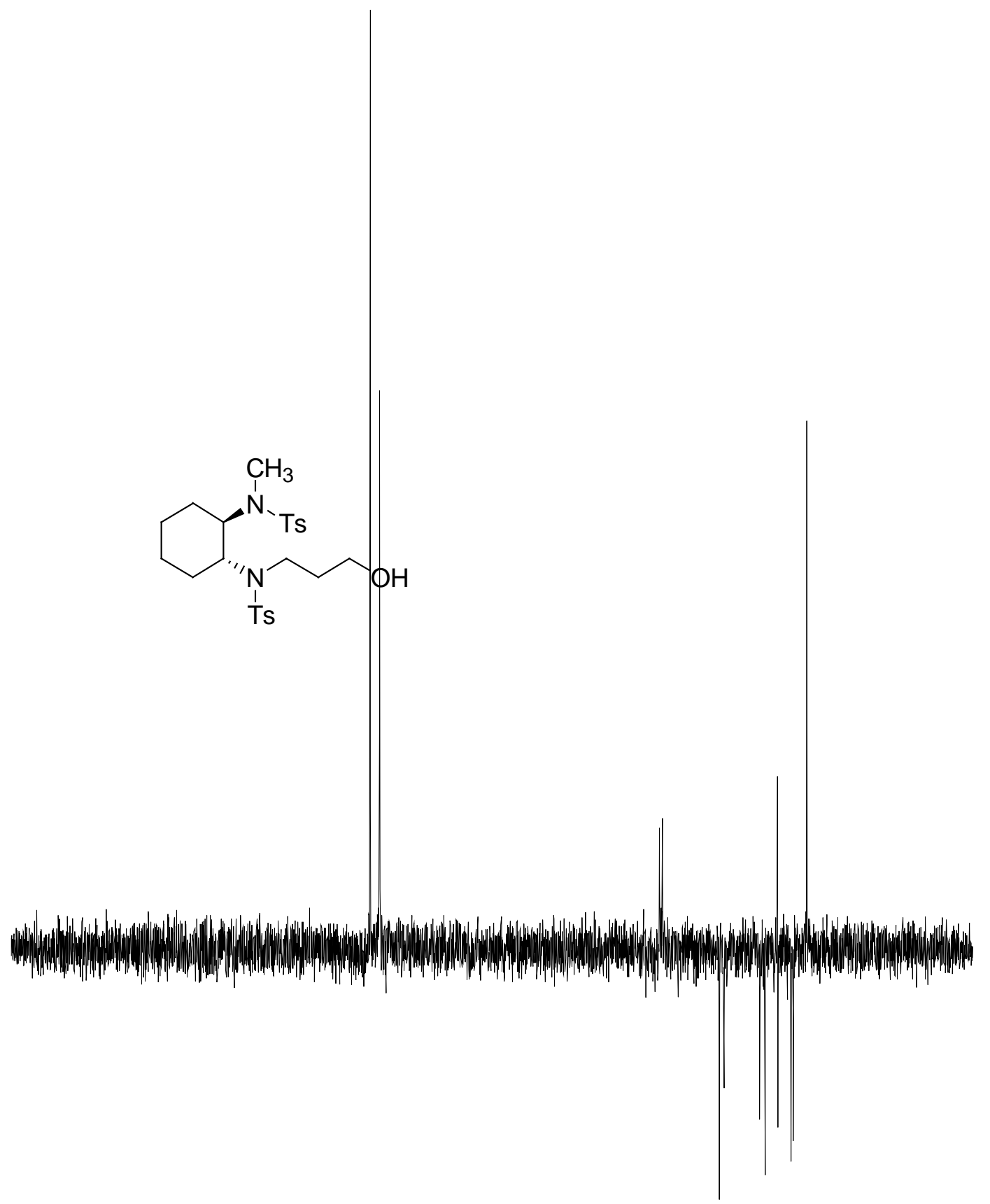

ताTाTा| 200

180

$160 \quad 140$

120

100

80

60

$40 \quad 20$

(ppom) 
${ }^{1} \mathrm{H}$ NMR spectrum of $(R, R)-5 \mathbf{a}\left(\mathrm{CDCl}_{3}, 300 \mathrm{MHz}\right)$

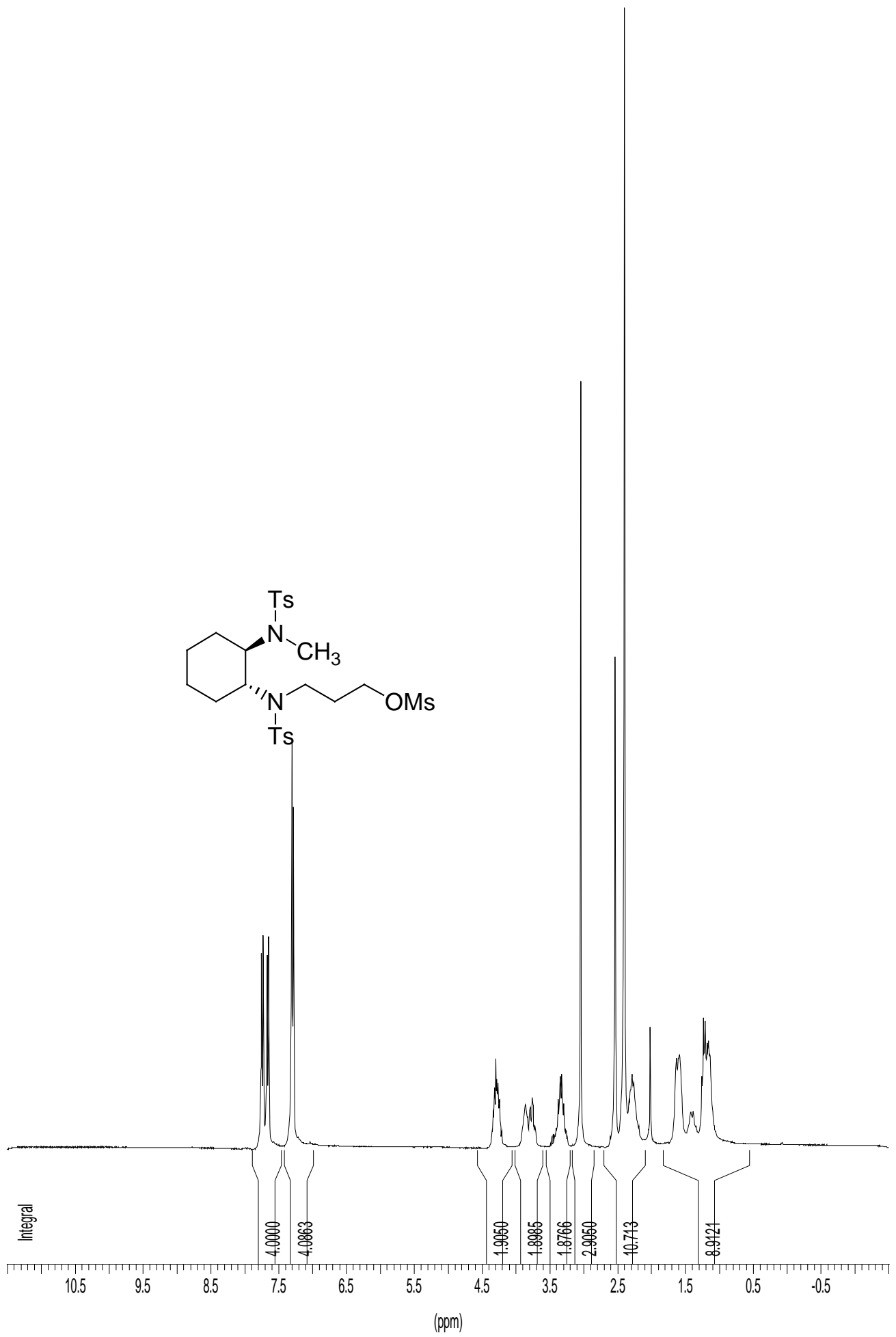


${ }^{13} \mathrm{C}$ NMR spectrum of $(R, R)-5 \mathbf{a}\left(\mathrm{CDCl}_{3}, 75 \mathrm{MHz}\right)$

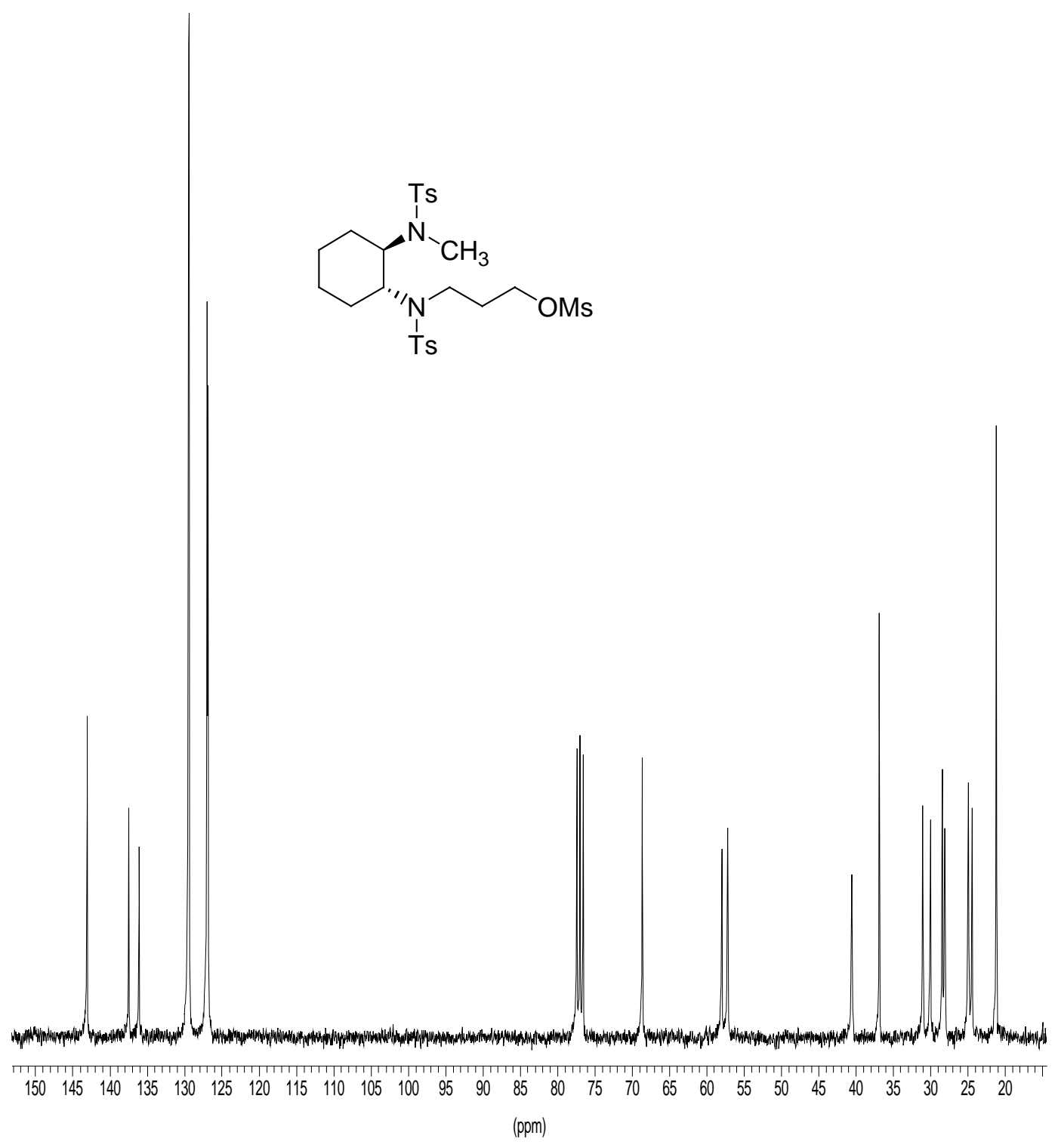


DEPT NMR spectrum of $(R, R)-5 \mathbf{a}\left(\mathrm{CDCl}_{3}, 75 \mathrm{MHz}\right)$

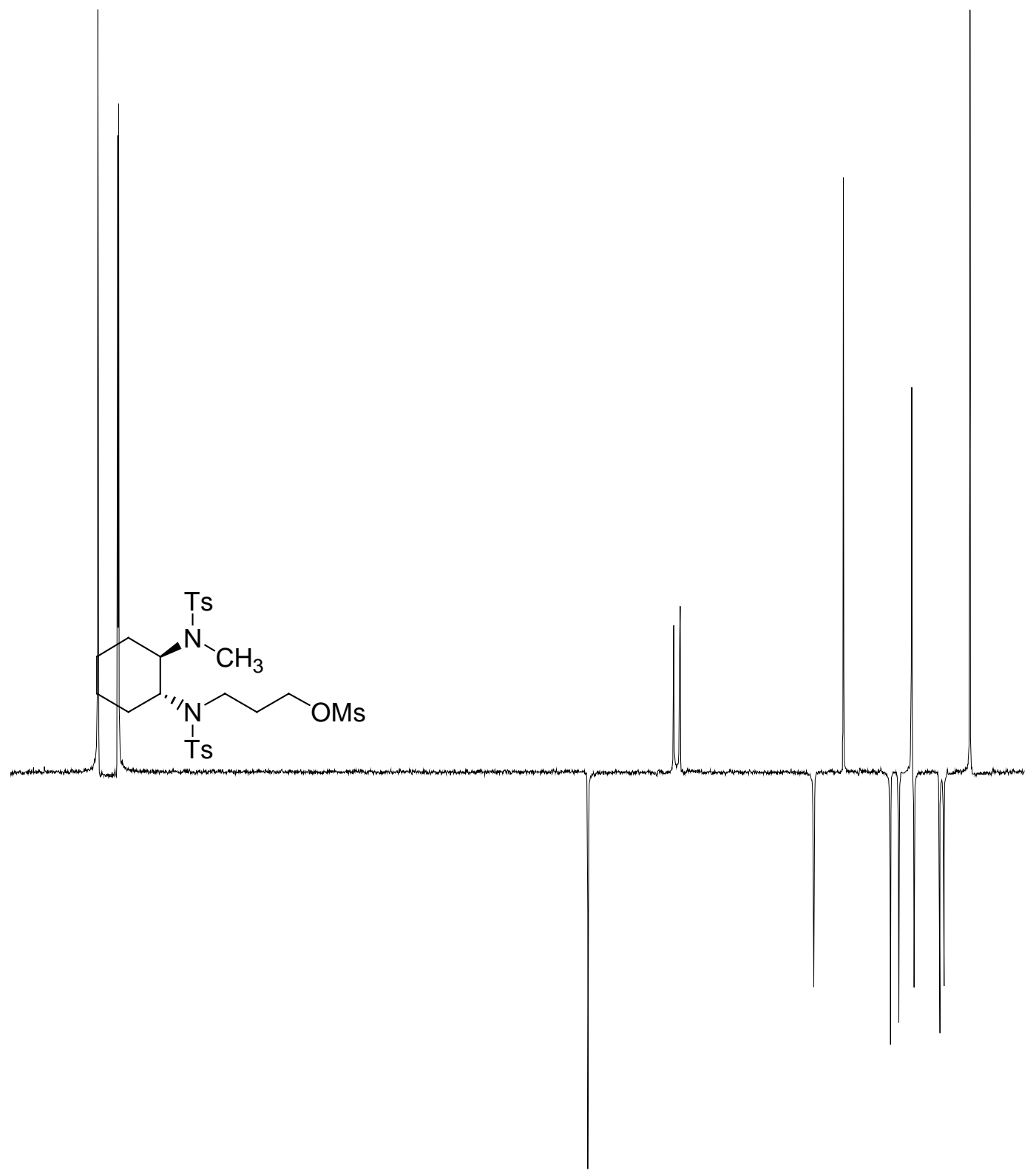

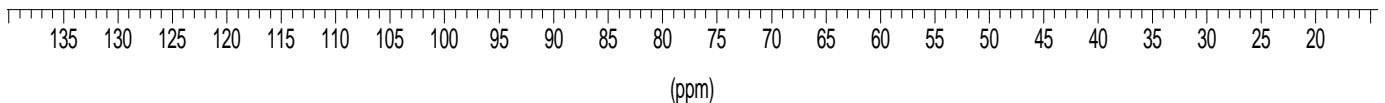


${ }^{1} \mathrm{H}$ NMR spectrum of $(R, R, R, R, R, R)-6 \mathbf{a}\left(\mathrm{CDCl}_{3}, 300 \mathrm{MHz}\right)$

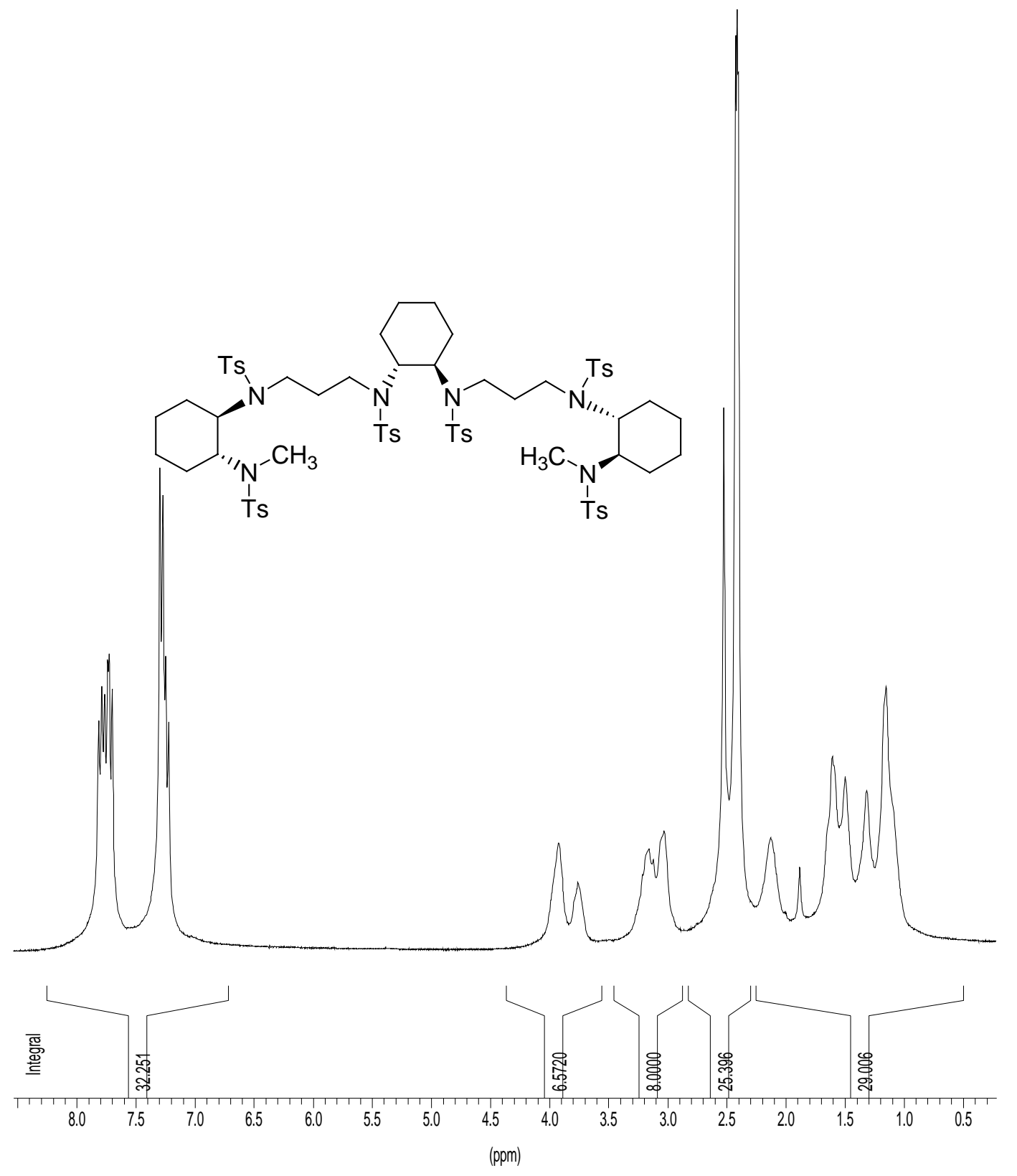


${ }^{13} \mathrm{C}$ NMR spectrum of $(R, R, R, R, R, R)-6 \mathbf{a}\left(\mathrm{CDCl}_{3}, 75 \mathrm{MHz}\right)$

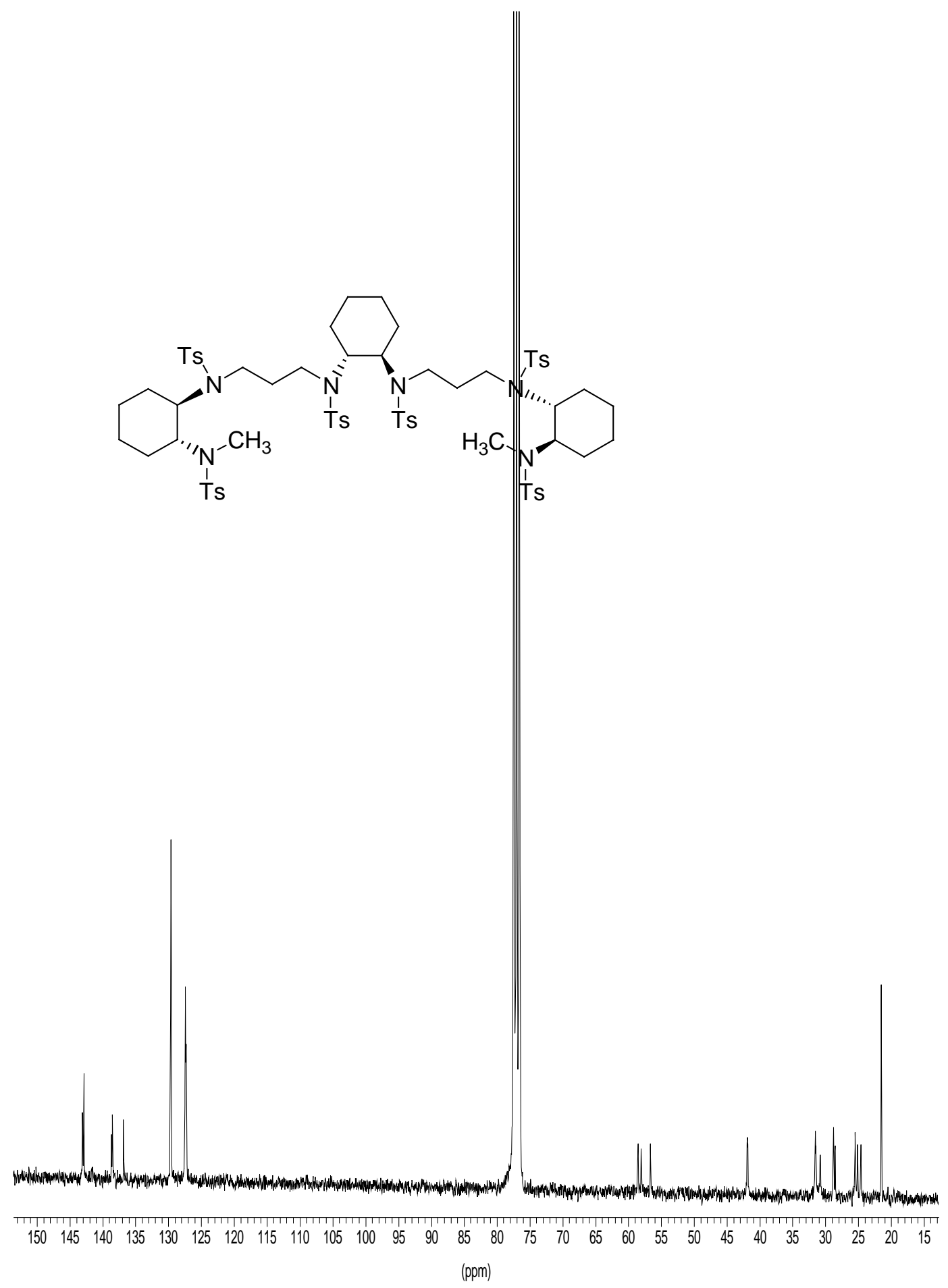


DEPT NMR spectrum of $(R, R, R, R, R, R)-6 \mathbf{a}\left(\mathrm{CDCl}_{3}, 75 \mathrm{MHz}\right)$
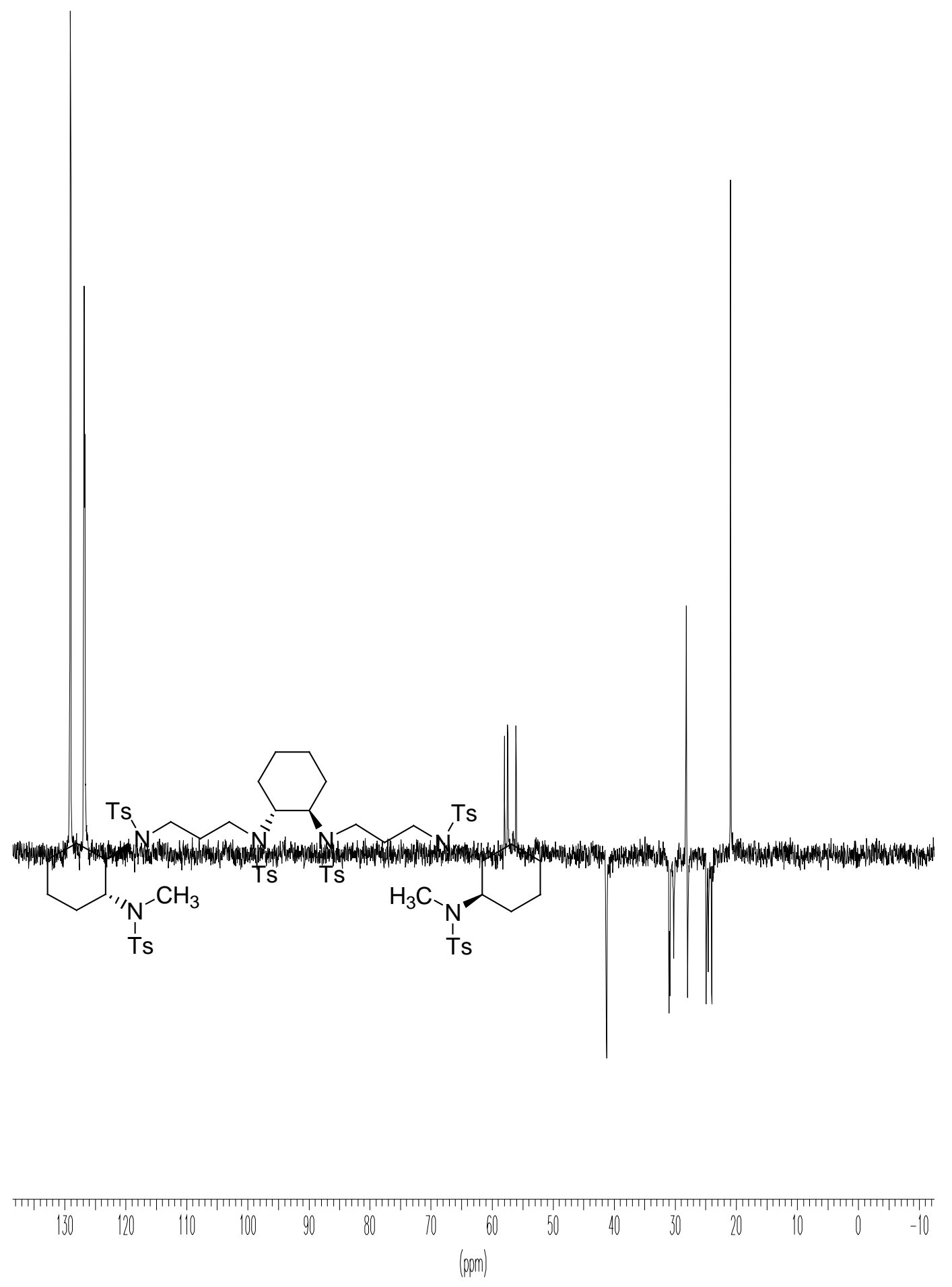

S-22 
${ }^{1} \mathrm{H}$ NMR spectrum of $(R, R, R, R, R, R)-7 \mathbf{a}\left(\mathrm{D}_{2} \mathrm{O}, 300 \mathrm{MHz}\right)$

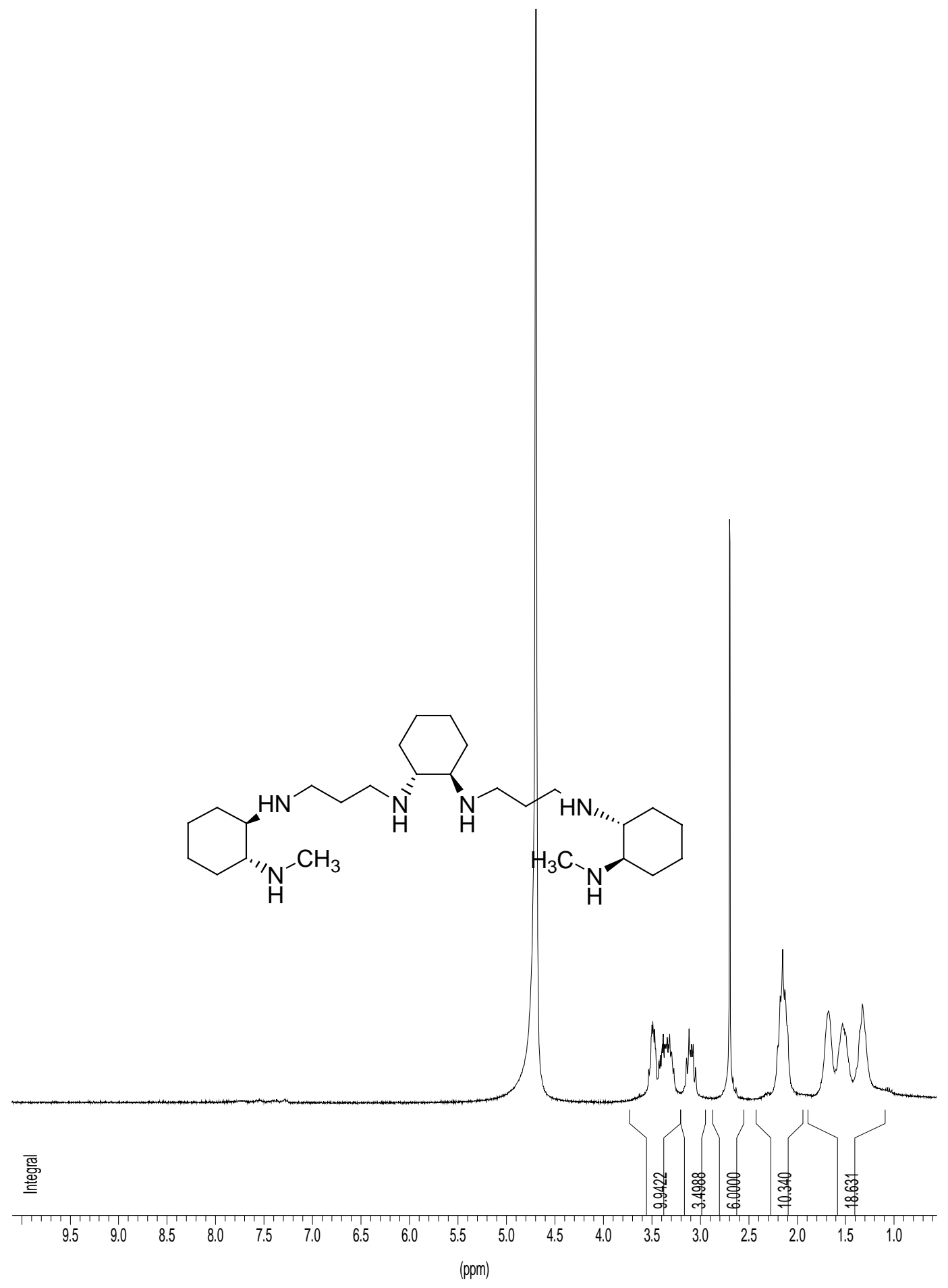


${ }^{13} \mathrm{C}$ NMR spectrum of $(R, R, R, R, R, R)-7 \mathbf{a}\left(\mathrm{D}_{2} \mathrm{O}, 75 \mathrm{MHz}\right)$
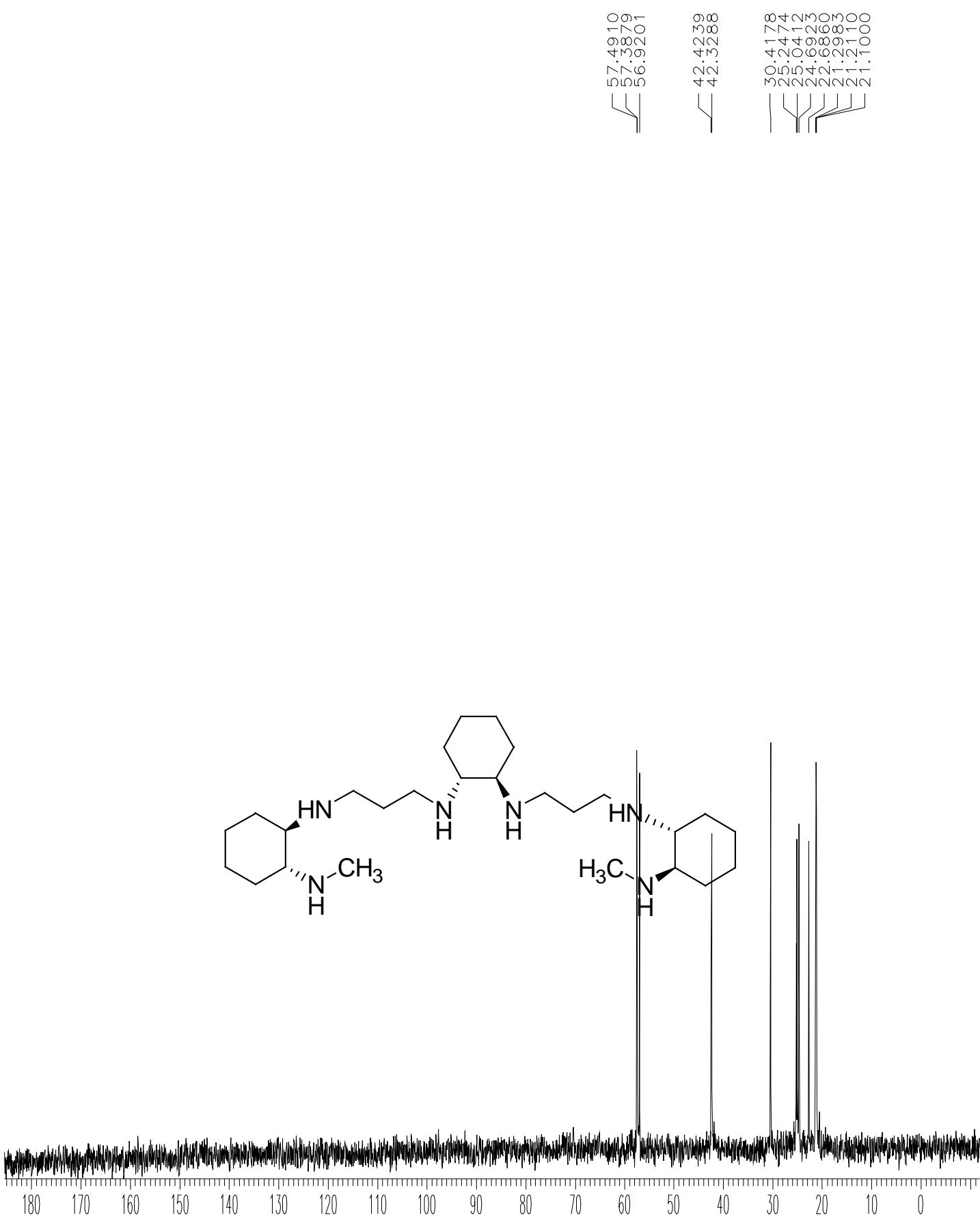

(pom) 
DEPT NMR spectrum of $(R, R, R, R, R, R)-7 \mathbf{a}\left(\mathrm{D}_{2} \mathrm{O}, 75 \mathrm{MHz}\right)$

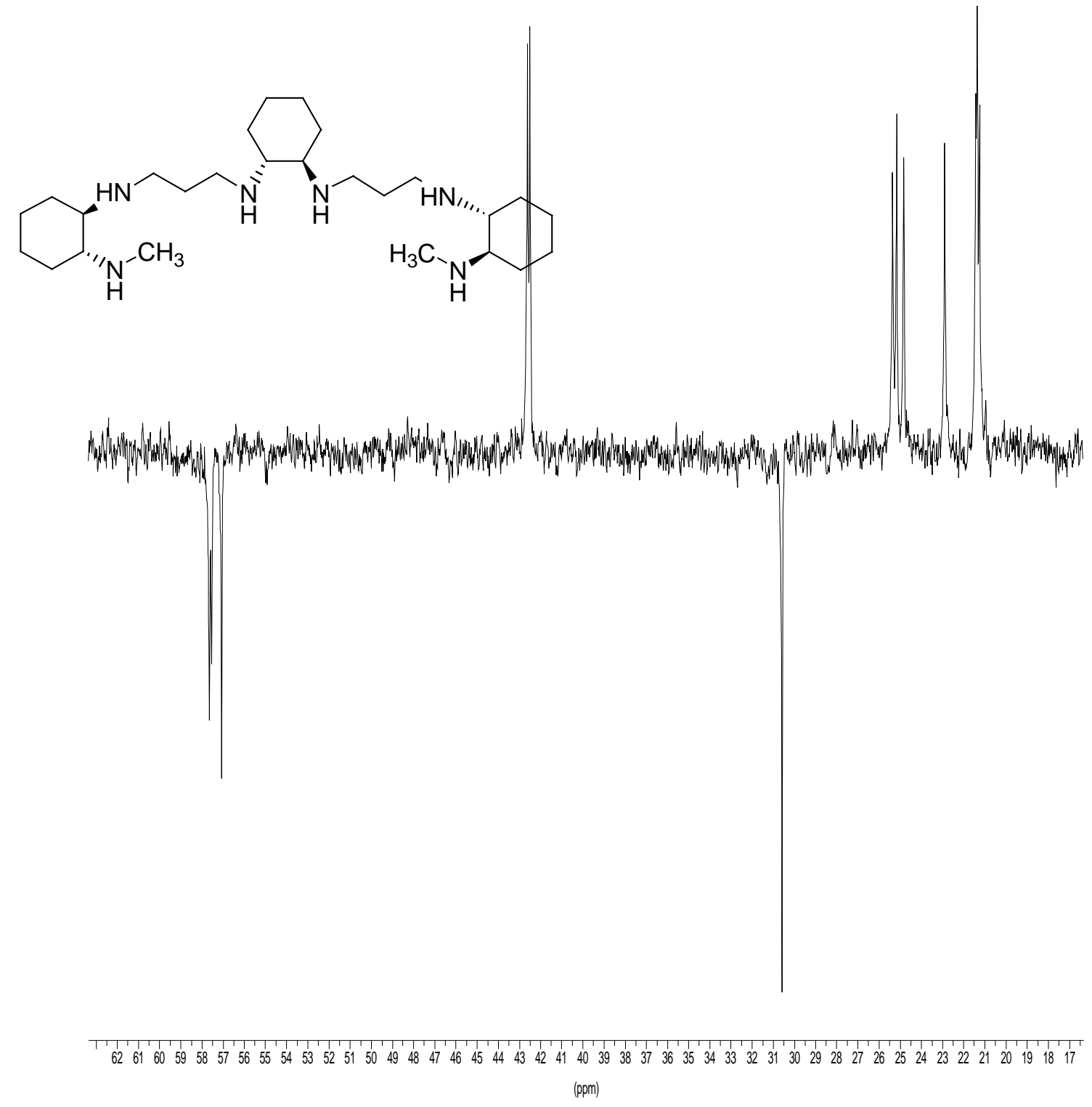


${ }^{1} \mathrm{H}$ NMR spectrum of $(S, S, R, R, S, S)-6 \mathbf{a}\left(\mathrm{CDCl}_{3}, 300 \mathrm{MHz}\right)$

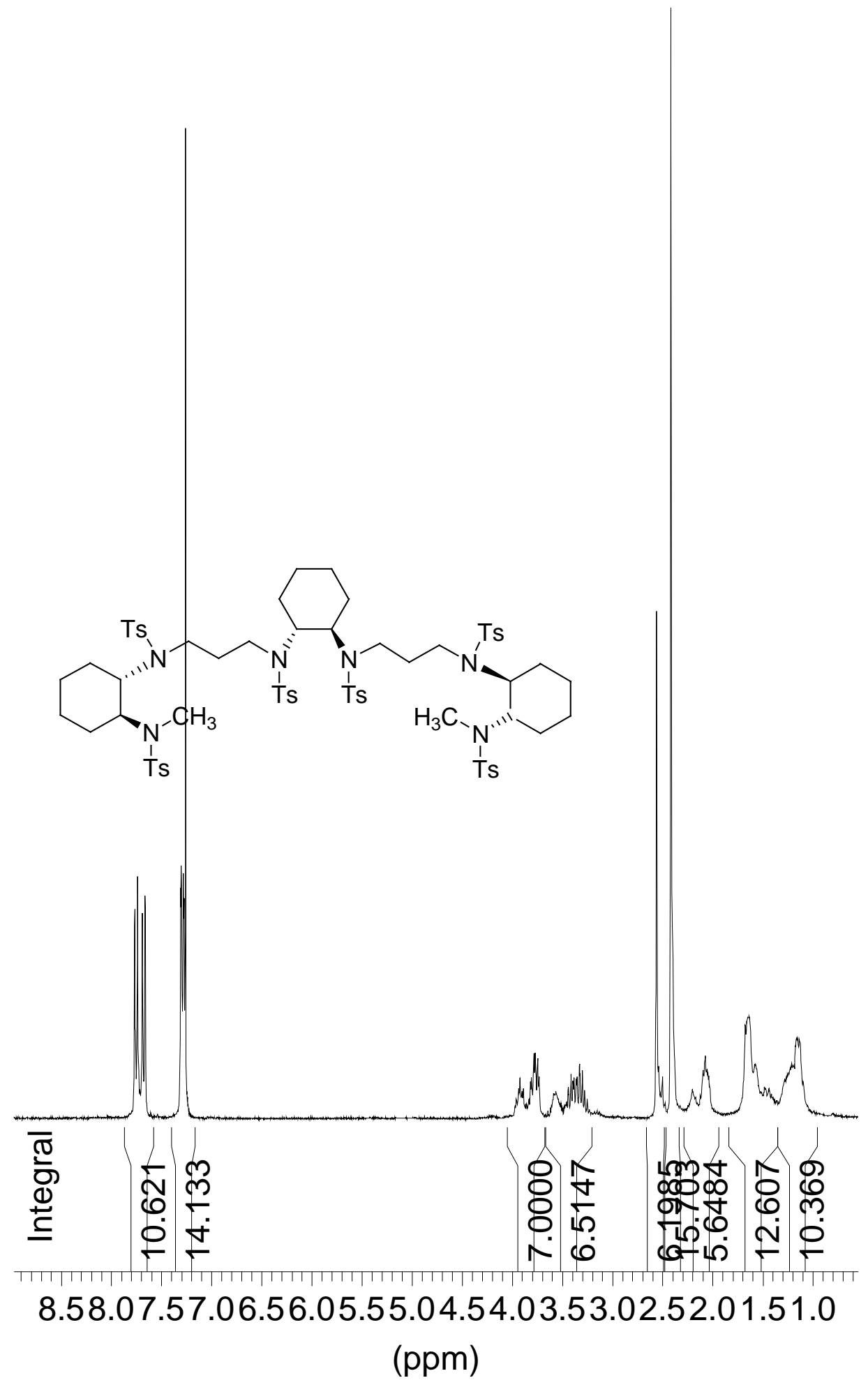


${ }^{13} \mathrm{C}$ NMR spectrum of $(S, S, R, R, S, S)-\mathbf{6 a}\left(\mathrm{CDCl}_{3}, 75 \mathrm{MHz}\right)$
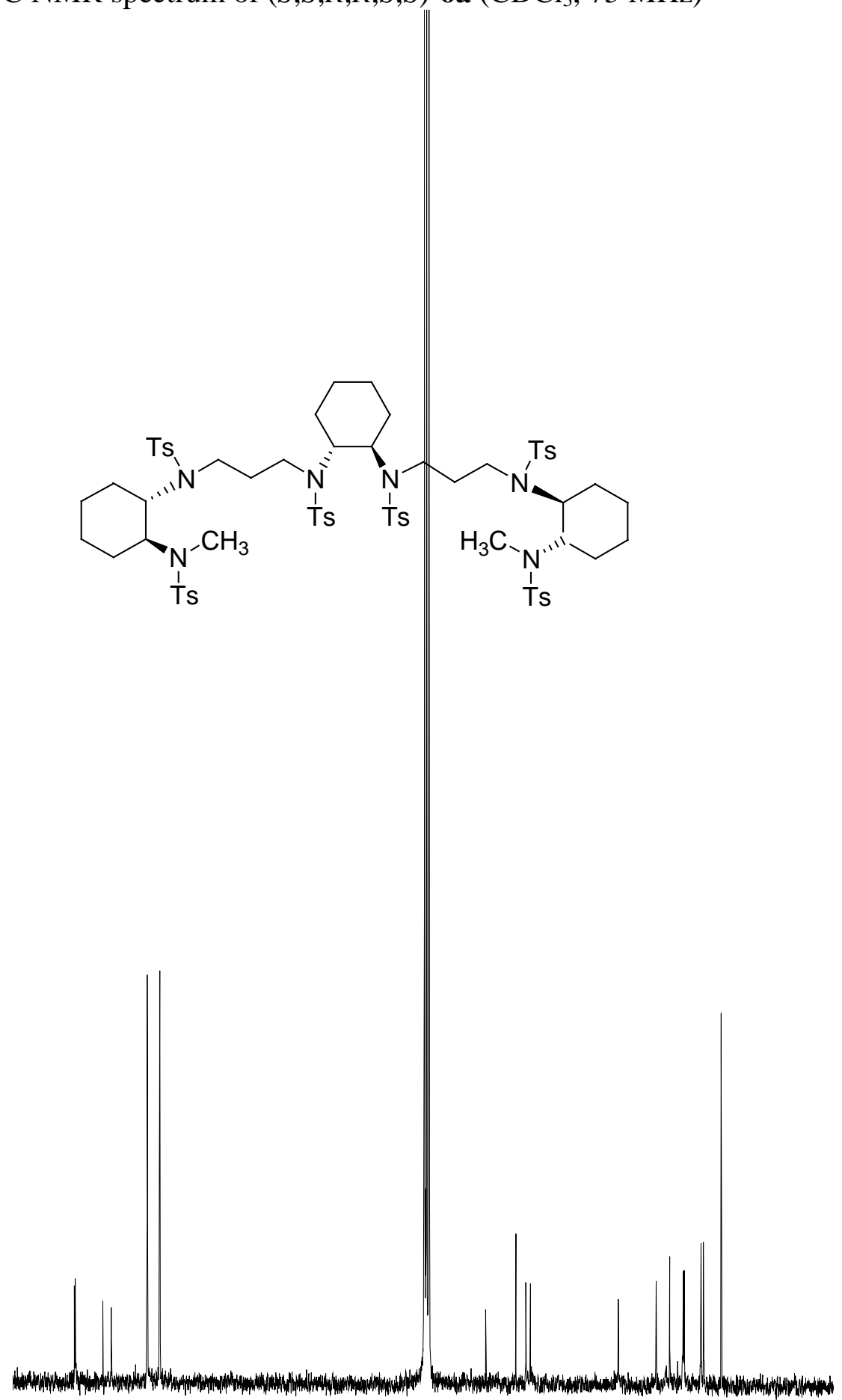

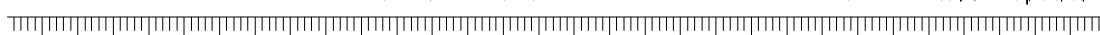

150140130120110100908070605040302010 (ppm) 
DEPT spectrum of $(S, S, R, R, S, S)-\mathbf{6 a}\left(\mathrm{CDCl}_{3}, 75 \mathrm{MHz}\right)$

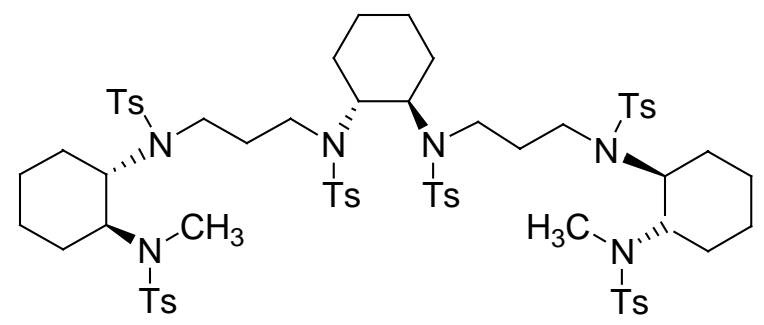

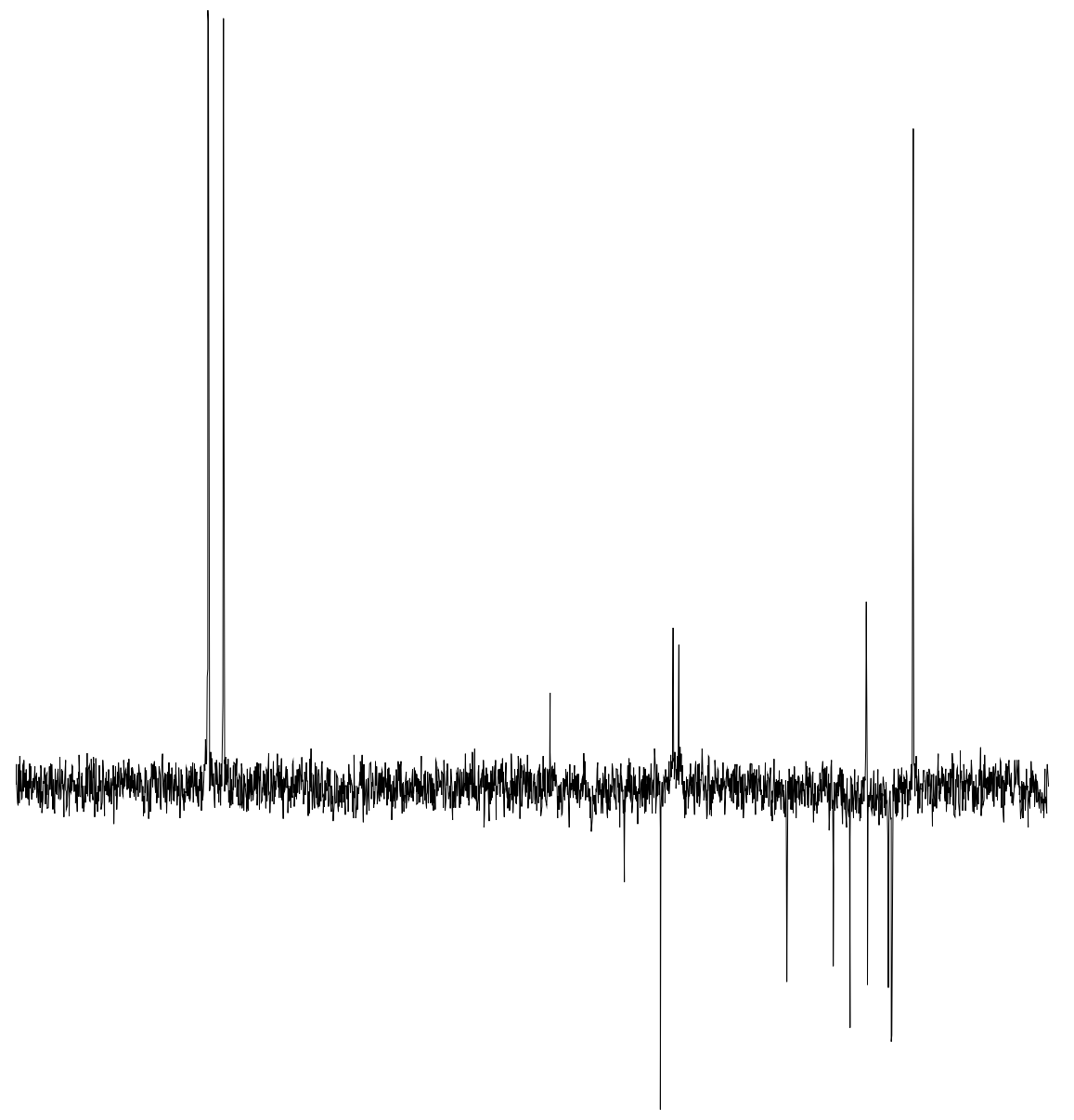

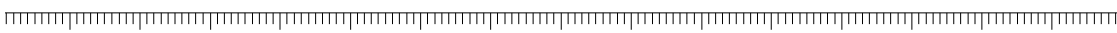
i0150140130120110100 $90 \quad 80 \quad 70 \quad 60 \quad 50 \quad 40 \quad 30 \quad 20 \quad 10$ (ppm) 
${ }^{1} \mathrm{H}$ NMR spectrum of $(S, S, R, R, S, S)-7 \mathbf{a}\left(\mathrm{D}_{2} \mathrm{O}, 300 \mathrm{MHz}\right)$

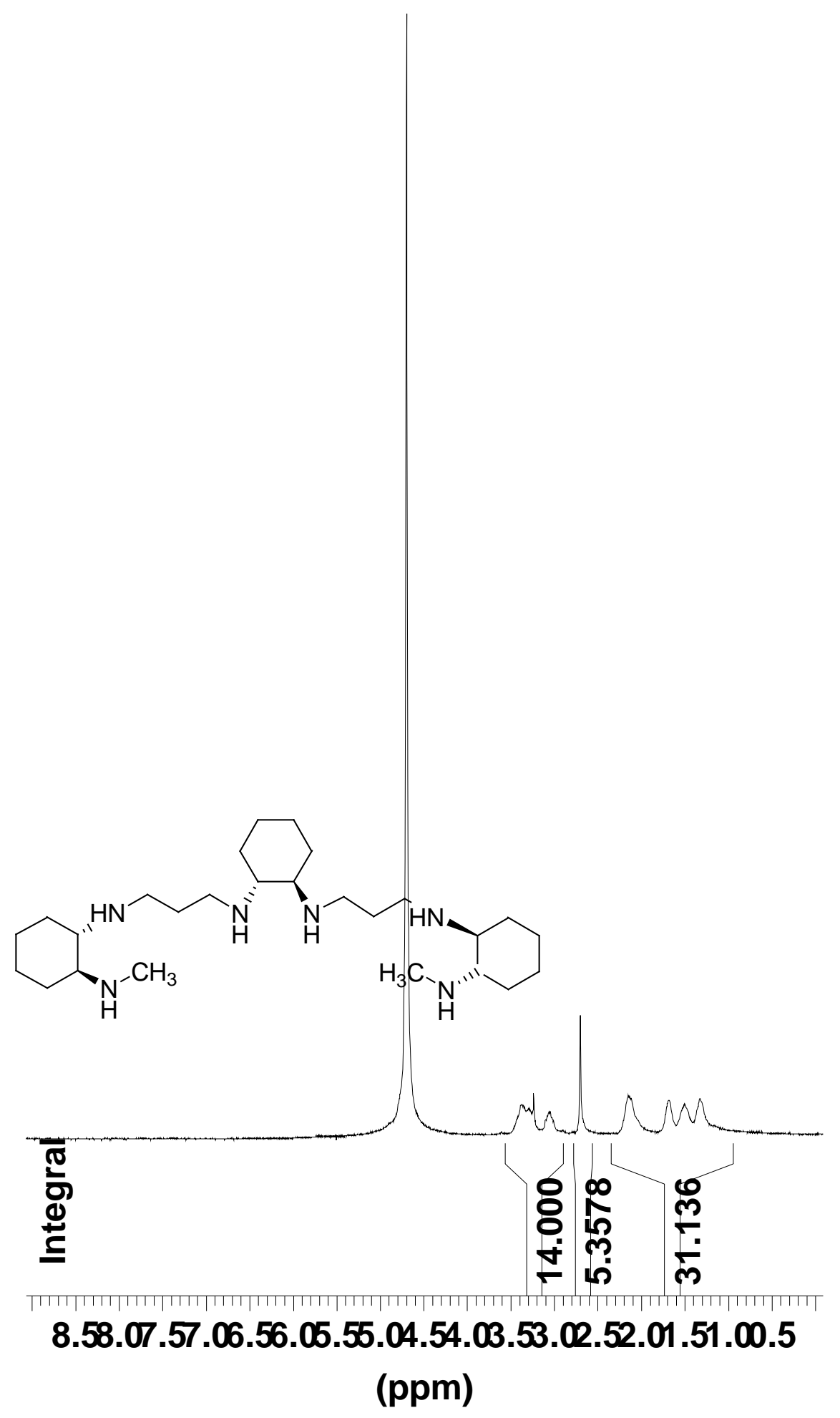


${ }^{13} \mathrm{C}$ NMR spectrum of $(S, S, R, R, S, S)-7 \mathbf{a}\left(\mathrm{D}_{2} \mathrm{O}, 75 \mathrm{MHz}\right)$

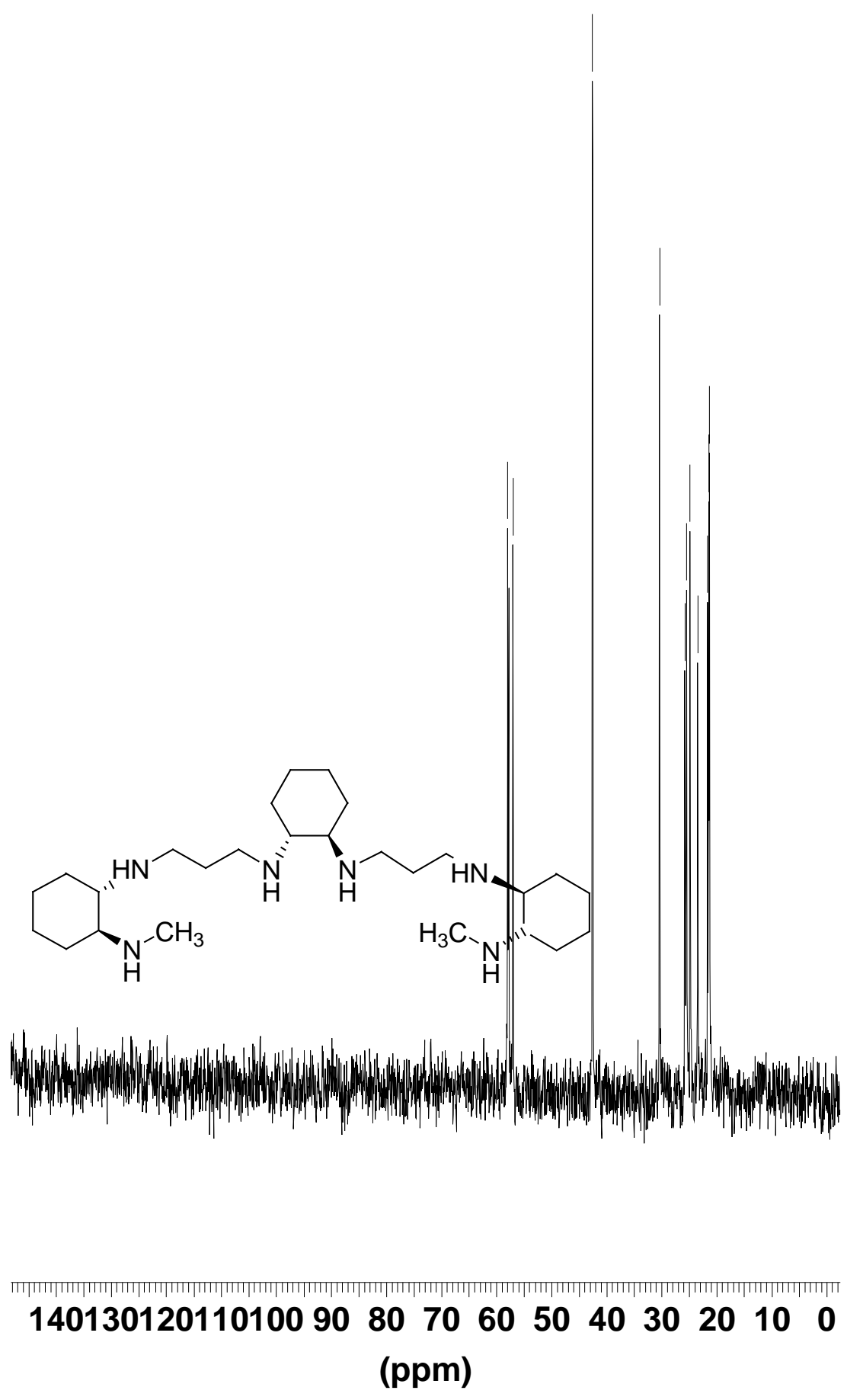


DEPT spectrum of $(S, S, R, R, S, S)-7 \mathbf{a}\left(\mathrm{D}_{2} \mathrm{O}, 75 \mathrm{MHz}\right)$<smiles>CN[C@H]1CCCC[C@@H]1NCCCN[C@@H]1CCCC[C@@H]1NCCCN[C@@H]1CCCC[C@@H]1NC</smiles>

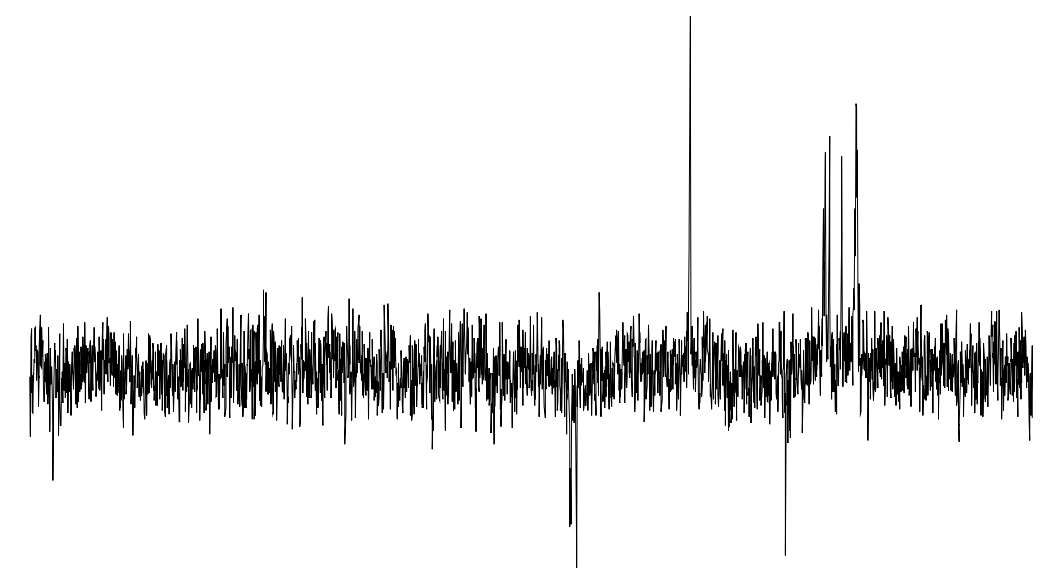

$\begin{array}{lllllllllll}120110100 & 90 & 80 & 70 & 60 & 50 & 40 & 30 & 20 & 10 & 0\end{array}$ (ppm) 
${ }^{1} \mathrm{H}$ NMR spectrum of $(R, R, R, R, R, R)-\mathbf{3 b}\left(\mathrm{CDCl}_{3}, 300 \mathrm{MHz}\right)$

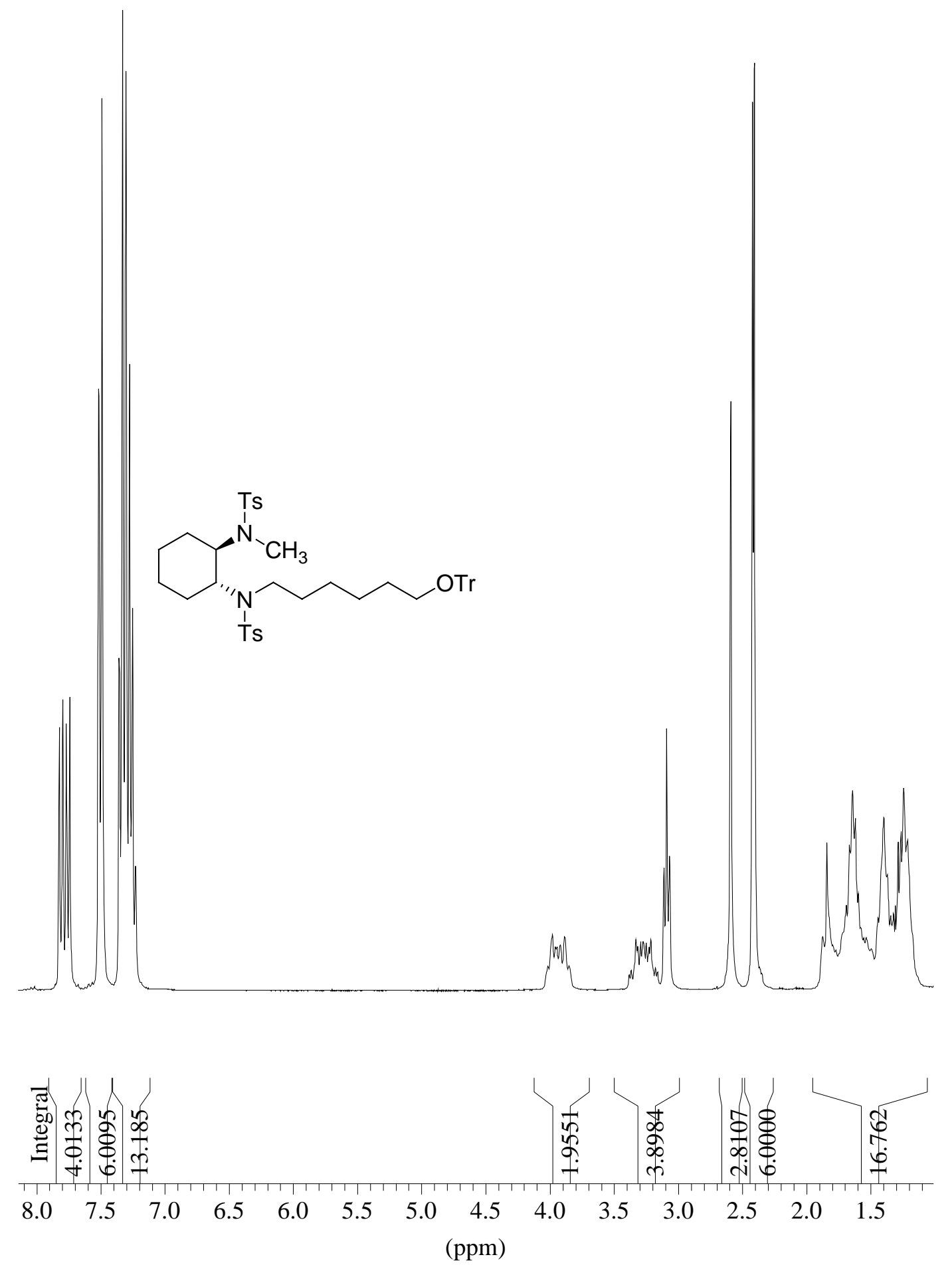


${ }^{13} \mathrm{C}$ NMR spectrum of $(R, R, R, R, R, R)-3 \mathbf{b}\left(\mathrm{CDCl}_{3}, 75 \mathrm{MHz}\right)$

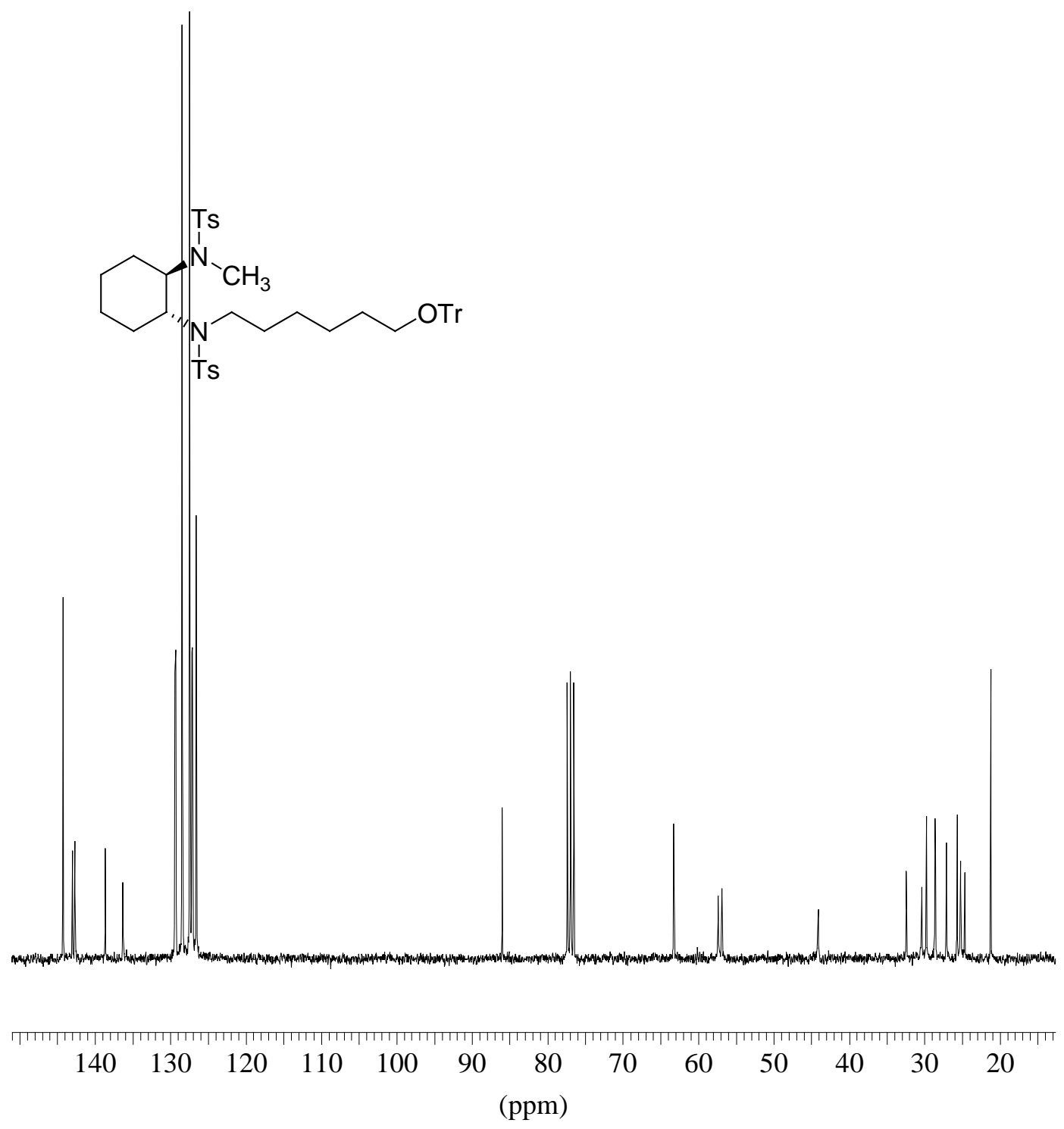


DEPT NMR spectrum of $(R, R, R, R, R, R)-3 \mathbf{b}\left(\mathrm{CDCl}_{3}, 75 \mathrm{MHz}\right)$

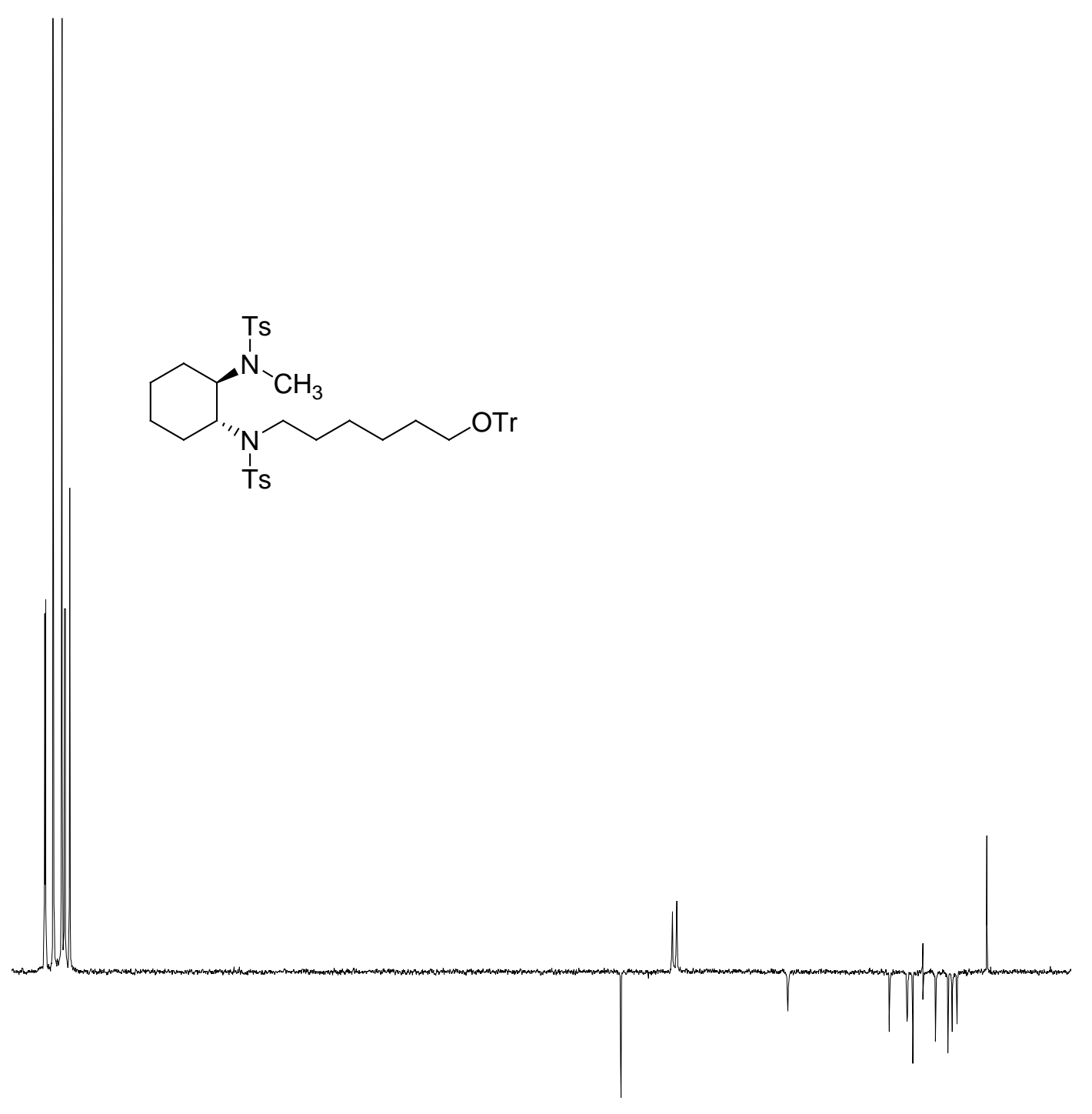
(ppm) 
${ }^{1} \mathrm{H}$ NMR spectrum of $(R, R, R, R, R, R)-\mathbf{4 b}\left(\mathrm{CDCl}_{3}, 300 \mathrm{MHz}\right)$

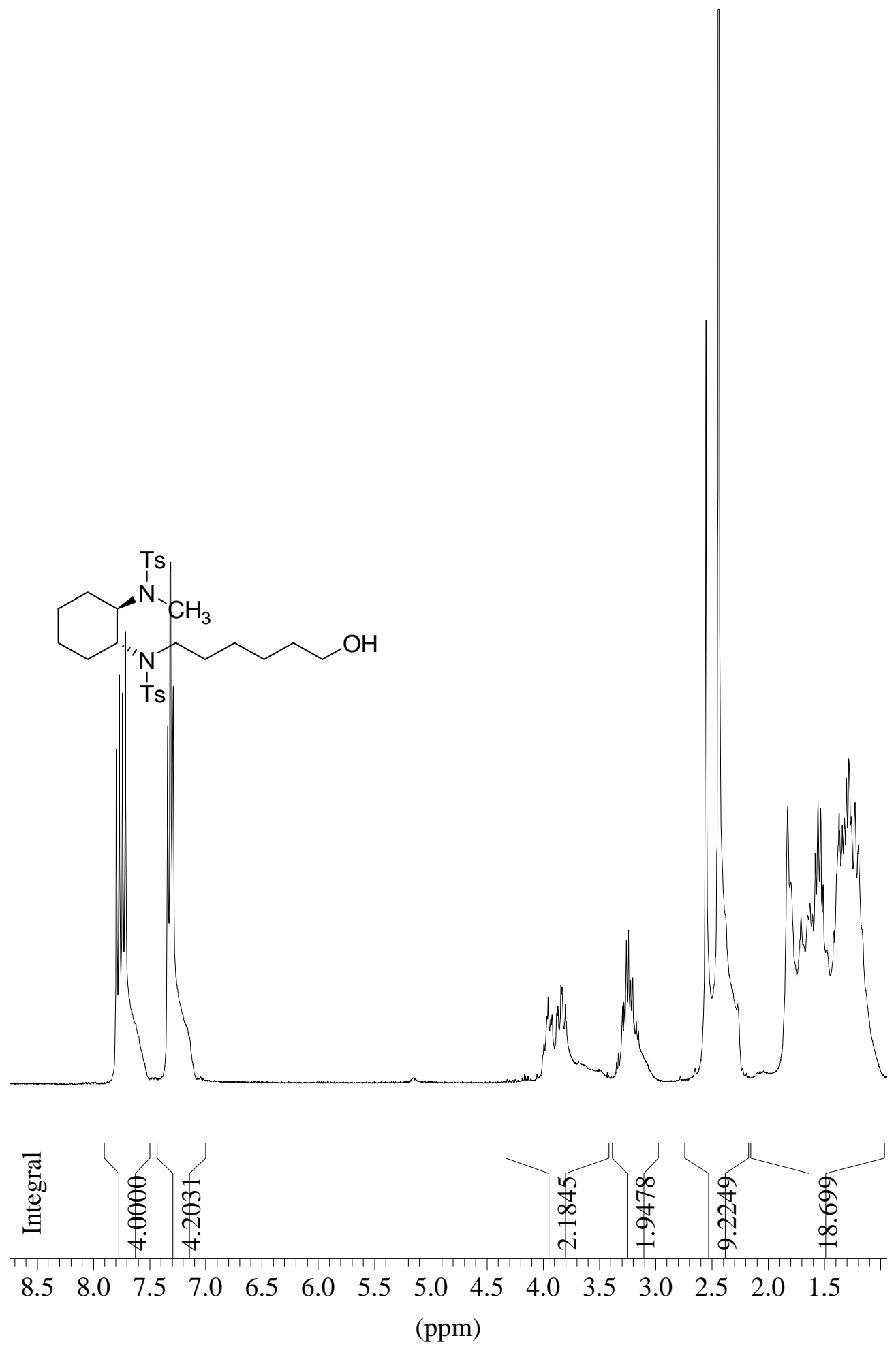


${ }^{13} \mathrm{C}$ NMR spectrum of $(R, R, R, R, R, R)-\mathbf{4 b}\left(\mathrm{CDCl}_{3}, 75 \mathrm{MHz}\right)$

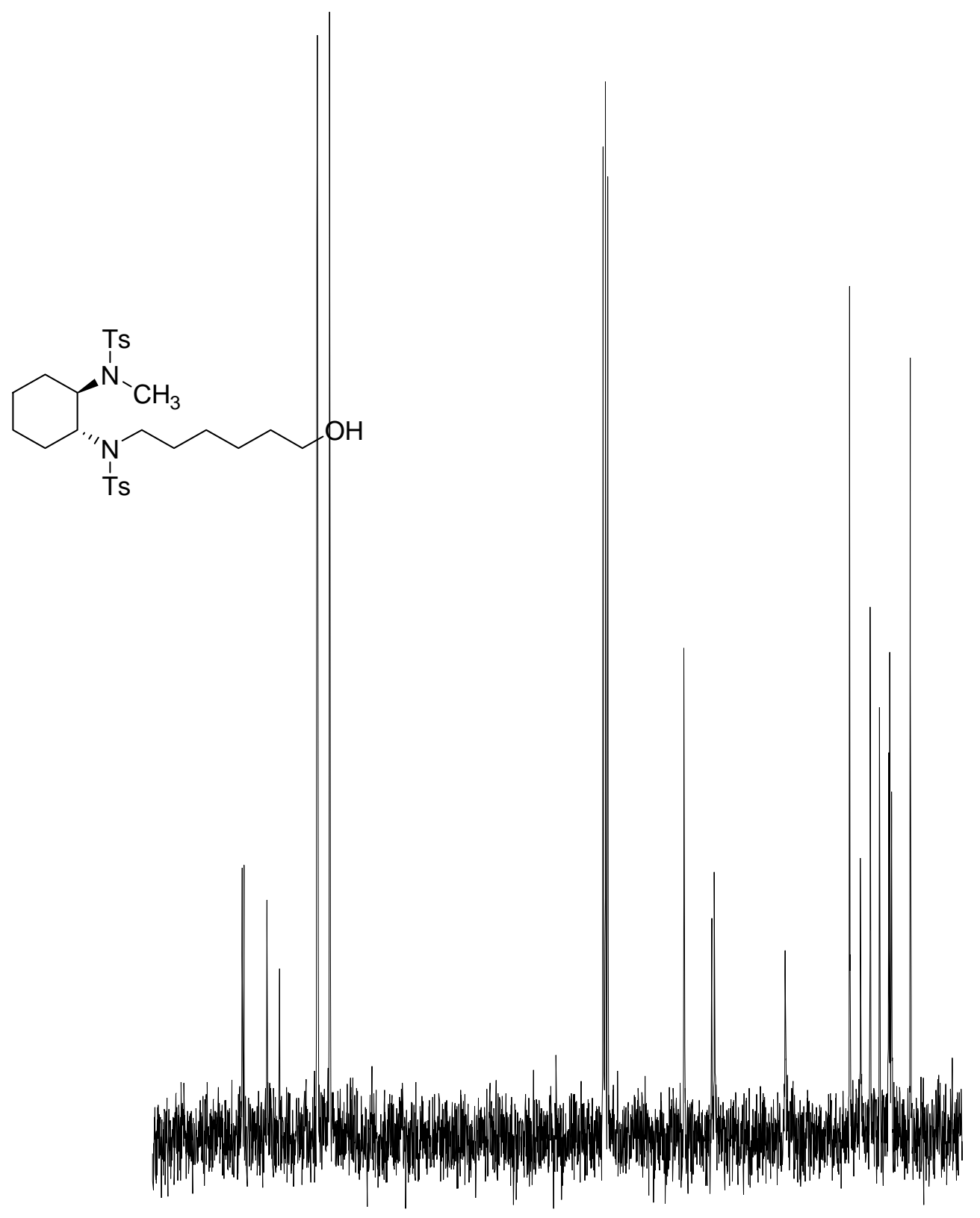

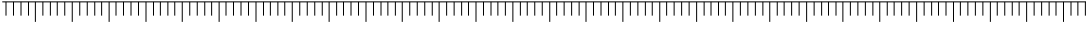

$\begin{array}{lllllllll}150140130120110100 & 90 & 80 & 70 & 60 & 50 & 40 & 30 & 20\end{array}$ (ppm)
} 
DEPT NMR spectrum of $(R, R, R, R, R, R)-\mathbf{4 b}\left(\mathrm{CDCl}_{3}, 75 \mathrm{MHz}\right)$

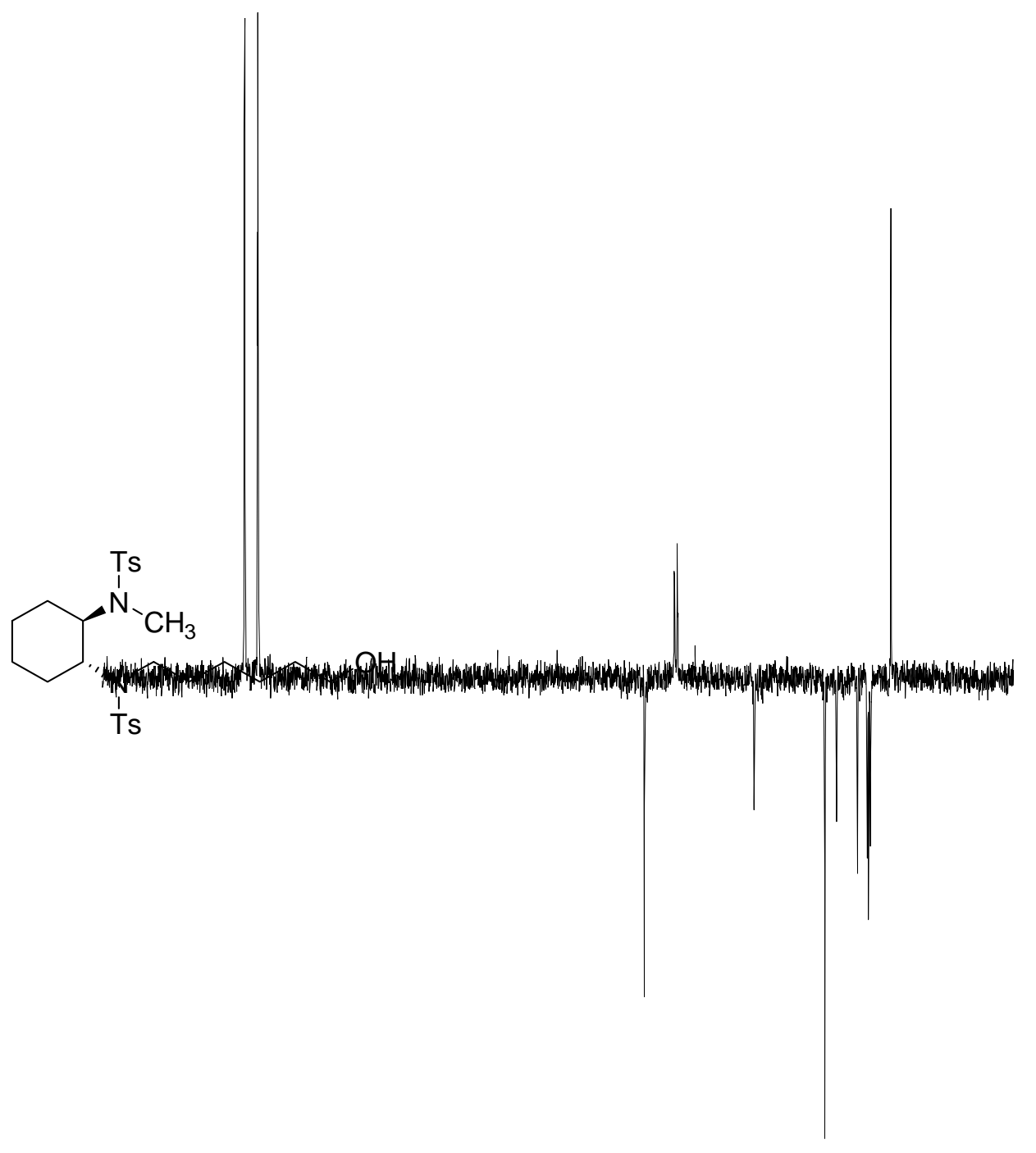

आ $15014013012011010090 \quad 80 \quad 70 \quad 60 \quad 50 \quad 40 \quad 30 \quad 20 \quad 10$ (ppm) 
${ }^{1} \mathrm{H}$ NMR spectrum of $(R, R, R, R, R, R)-5 \mathbf{b}\left(\mathrm{CDCl}_{3}, 300 \mathrm{MHz}\right)$

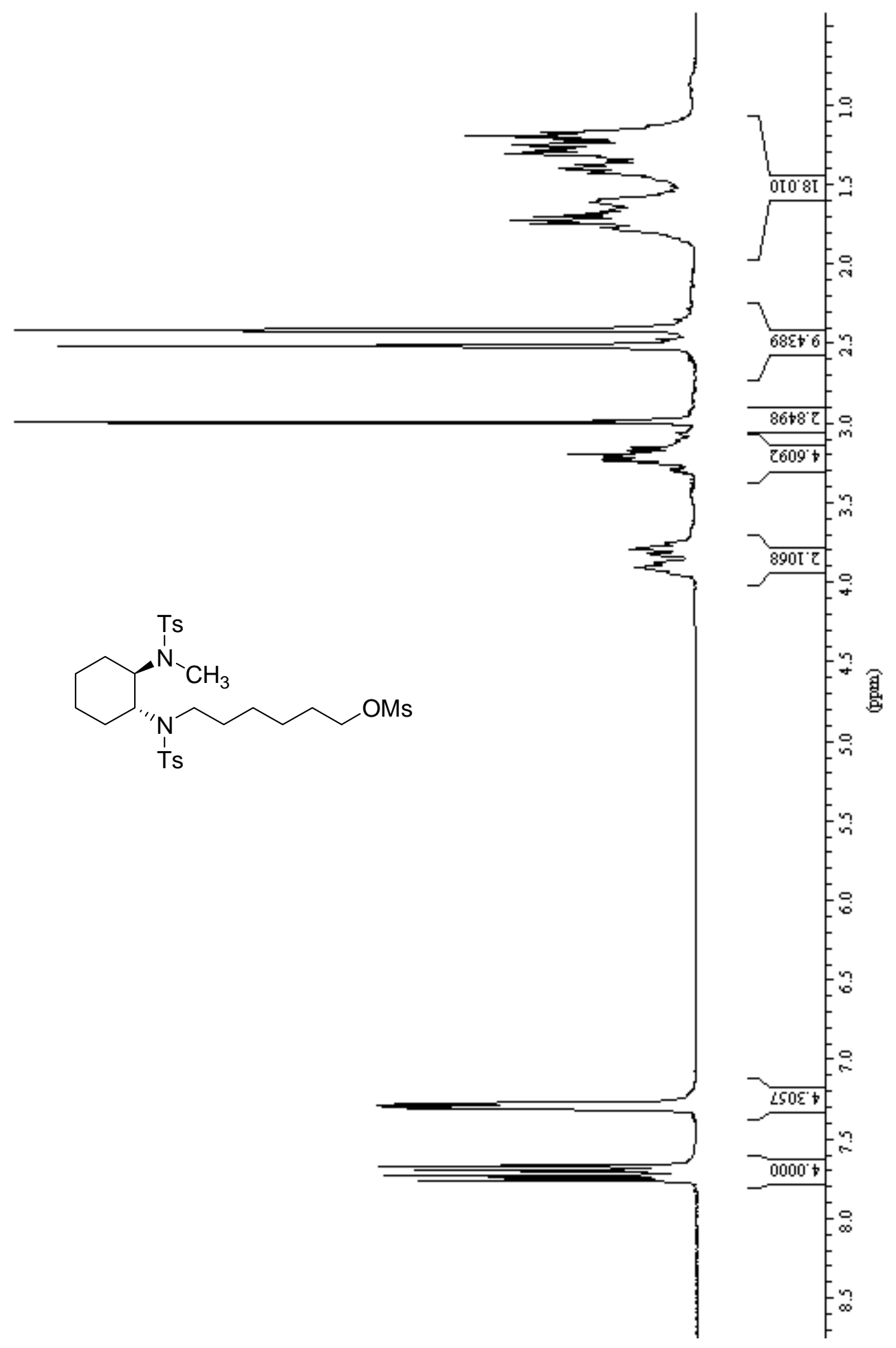


${ }^{13} \mathrm{C}$ NMR spectrum of $(R, R, R, R, R, R)-5 \mathbf{b}\left(\mathrm{CDCl}_{3}, 75 \mathrm{MHz}\right)$

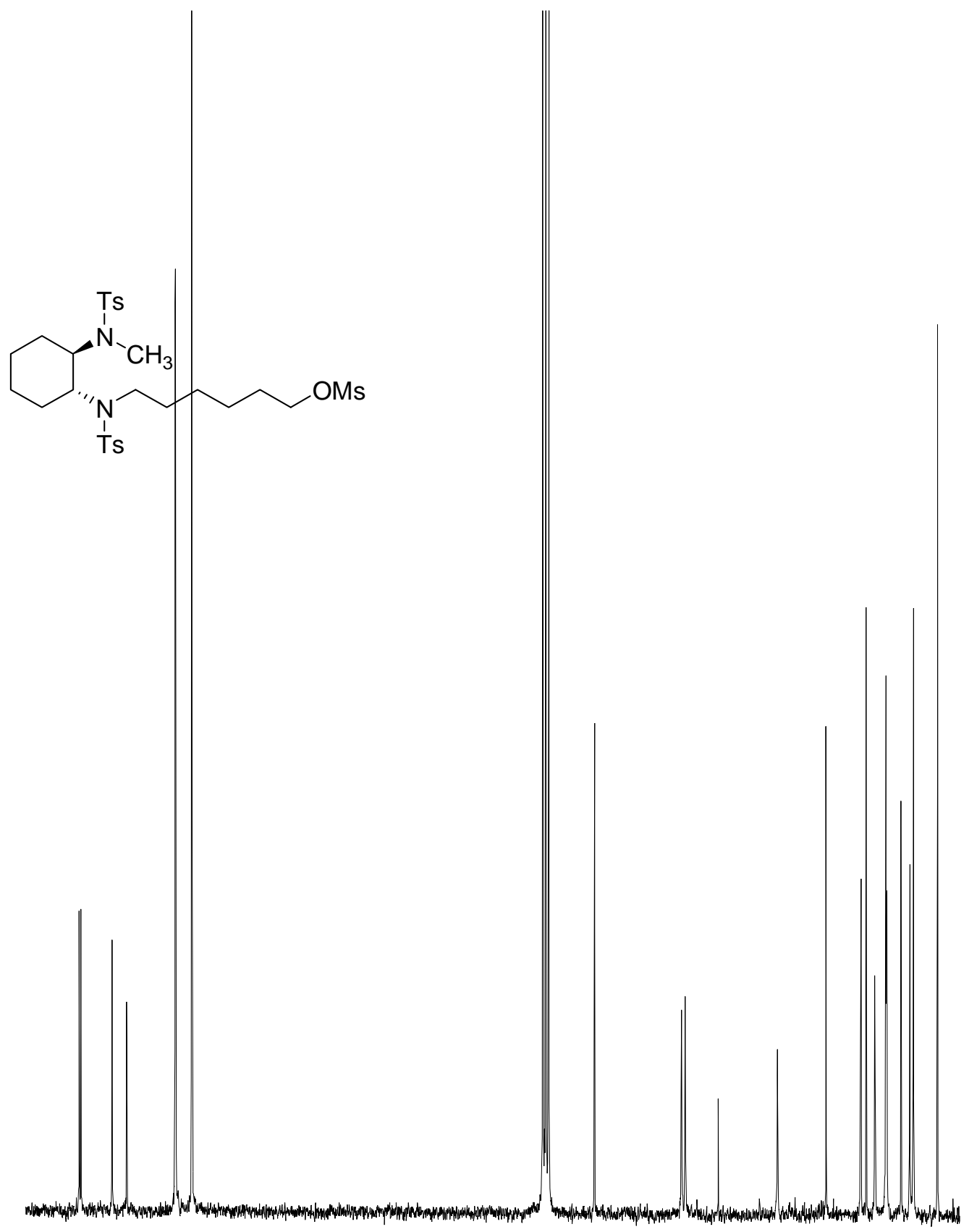

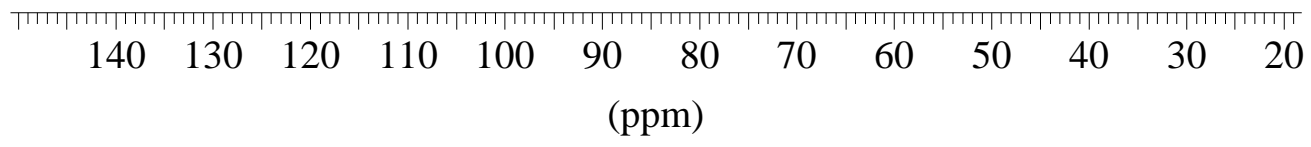


DEPT NMR spectrum of $(R, R, R, R, R, R)-5 \mathbf{b}\left(\mathrm{CDCl}_{3}, 75 \mathrm{MHz}\right)$

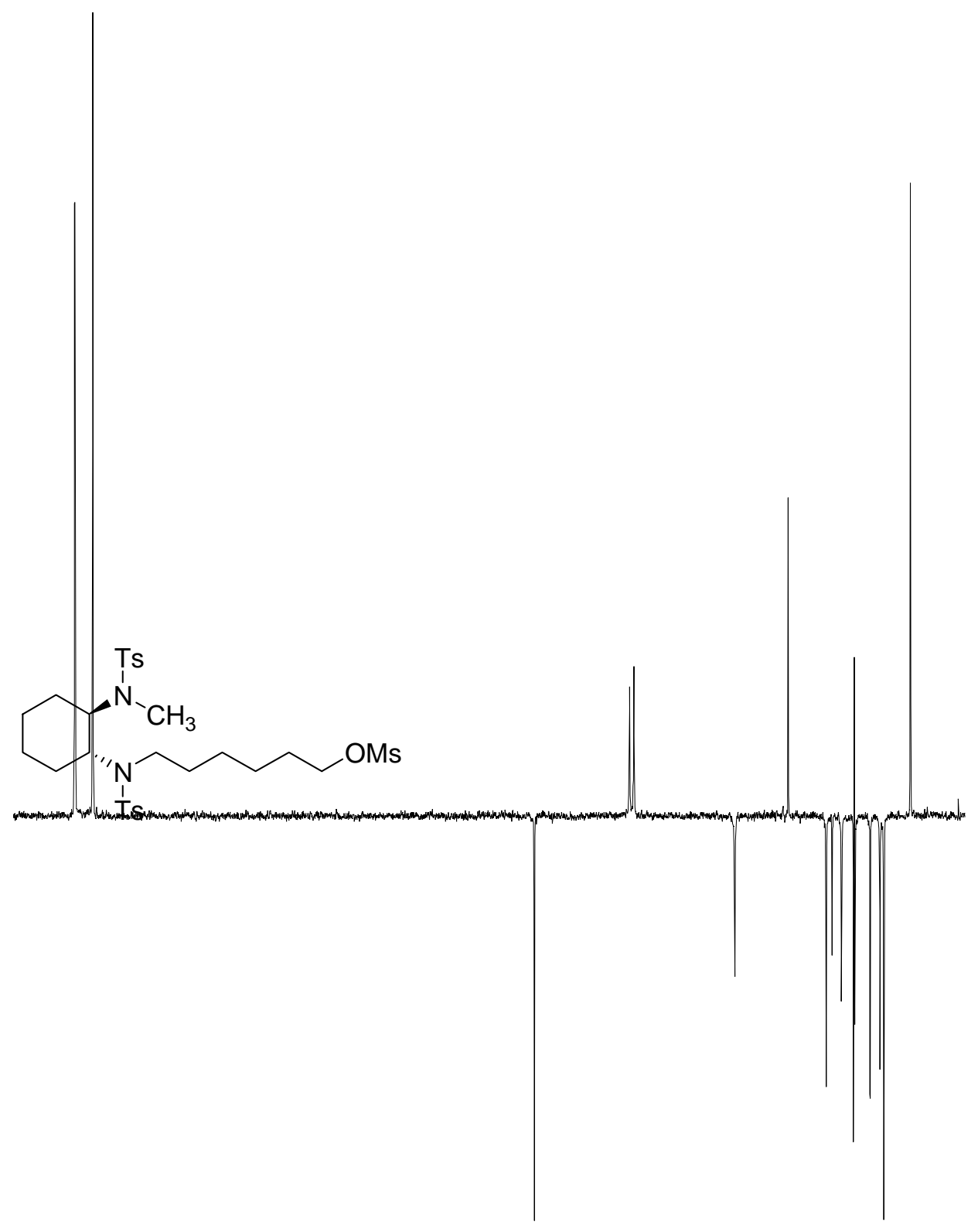

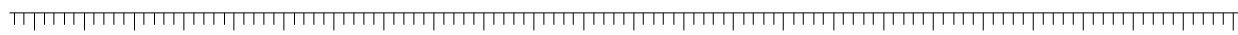
1353025201510050095908580757065605550454035302520 (ppm) 
${ }^{1} \mathrm{H}$ NMR spectrum of $(R, R, R, R, R, R)-6 \mathbf{b}\left(\mathrm{CDCl}_{3}, 300 \mathrm{MHz}\right)$

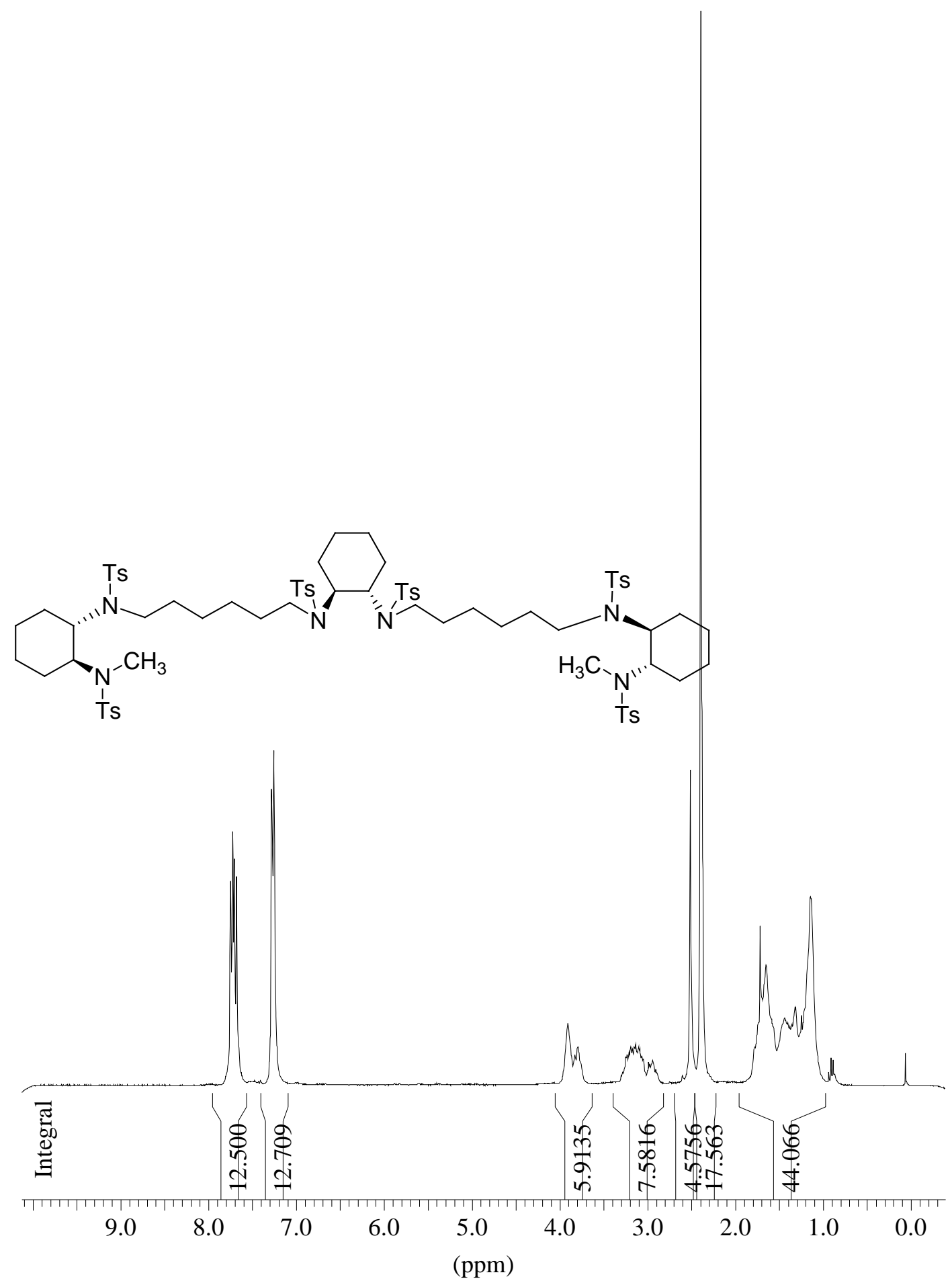


${ }^{13} \mathrm{C}$ NMR spectrum of $(R, R, R, R, R, R)-6 \mathbf{b}\left(\mathrm{CDCl}_{3}, 75 \mathrm{MHz}\right)$

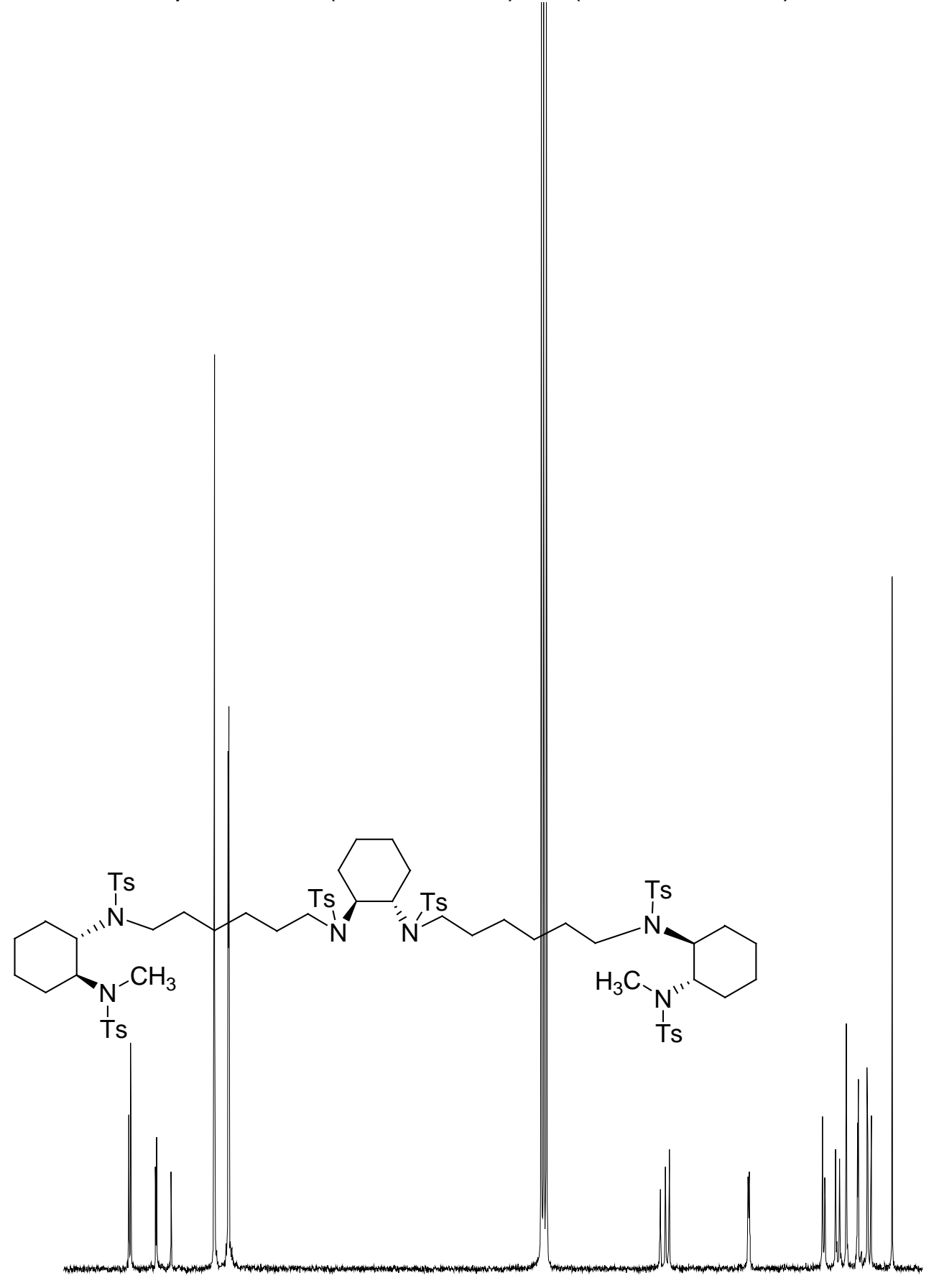

TाTाTा|

$150140130120110100 \quad 90 \quad 80 \quad 70 \quad 60 \quad 50 \quad 40 \quad 30 \quad 20$

(ppm) 
DEPT NMR spectrum of $(R, R, R, R, R, R)-6 \mathbf{b}\left(\mathrm{CDCl}_{3}, 75 \mathrm{MHz}\right)$

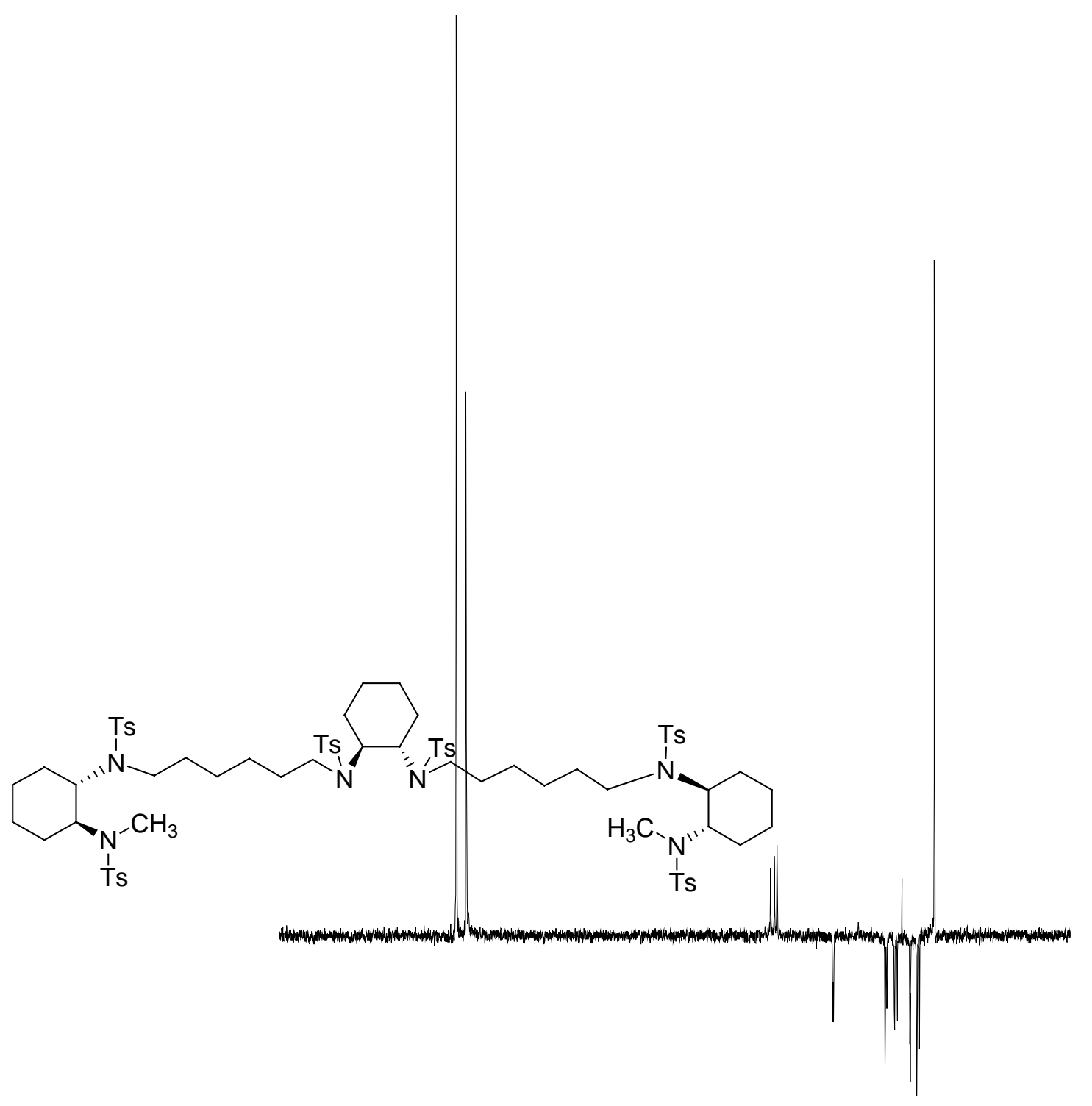

(ppm) 
${ }^{1} \mathrm{H}$ NMR spectrum of $(R, R, R, R, R, R)-7 \mathbf{b}\left(\mathrm{D}_{2} \mathrm{O}, 300 \mathrm{MHz}\right)$

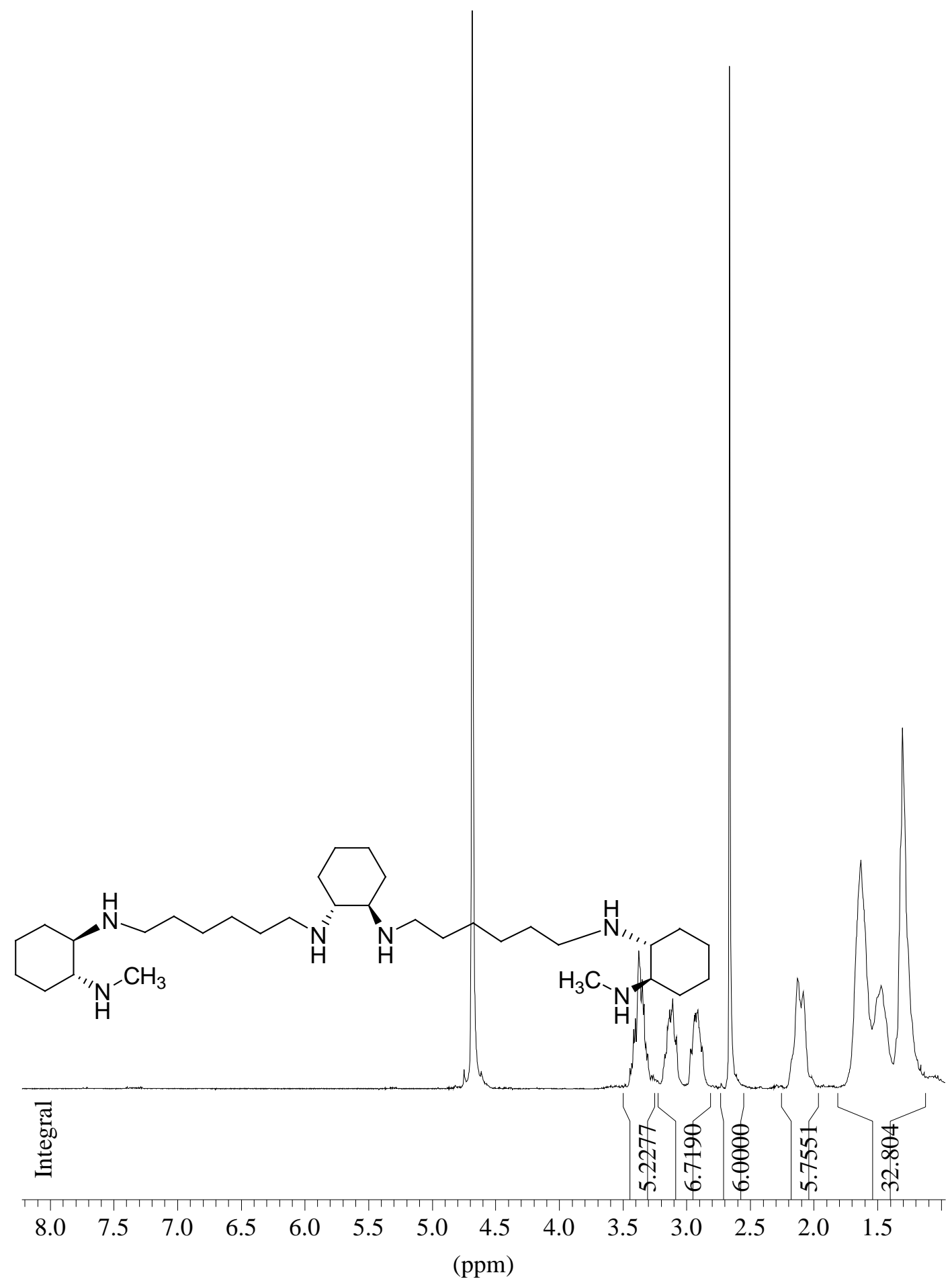


${ }^{13} \mathrm{C}$ NMR spectrum of $(R, R, R, R, R, R)-7 \mathbf{b}\left(\mathrm{D}_{2} \mathrm{O}, 75 \mathrm{MHz}\right)$

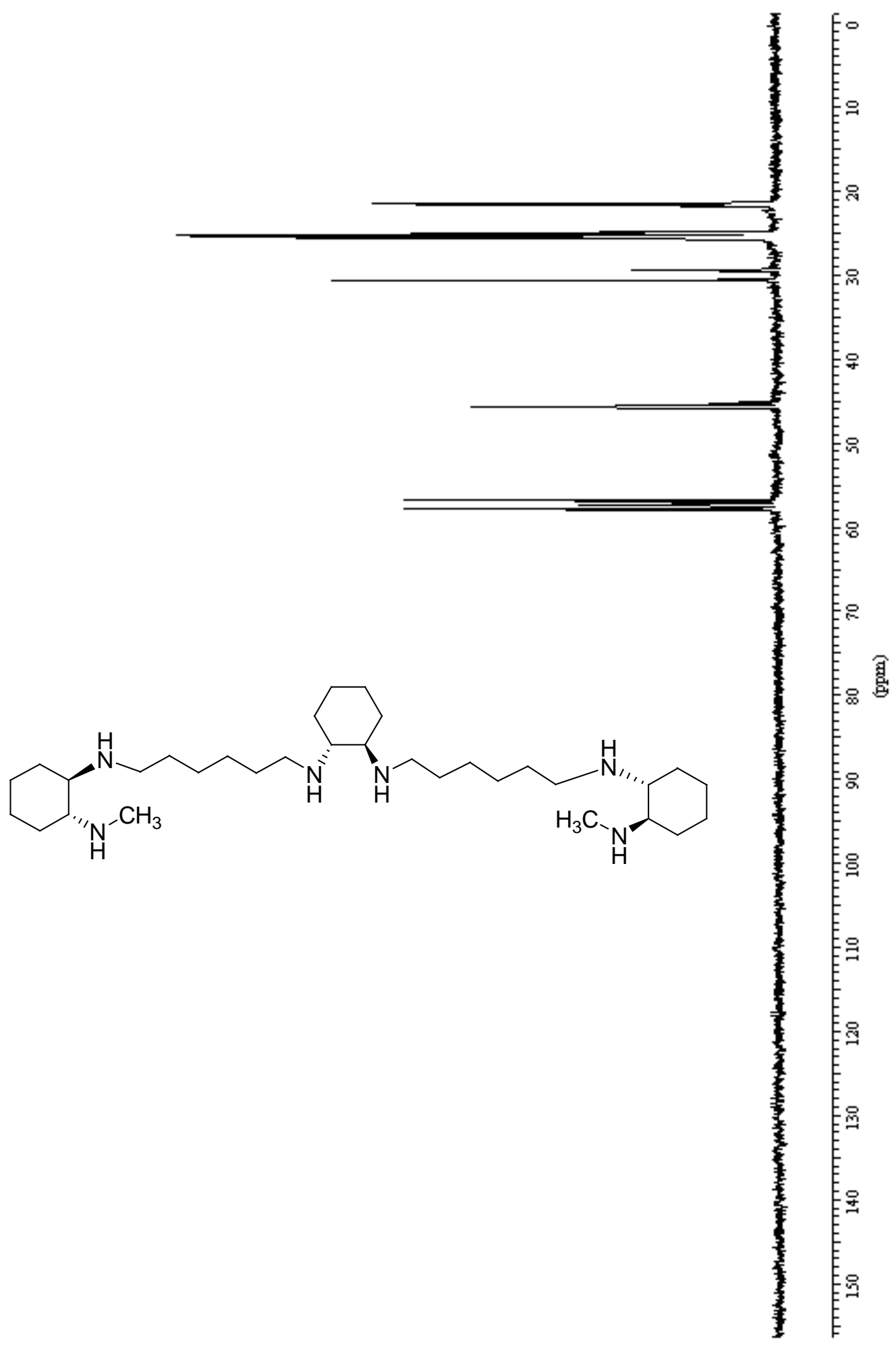


DEPT NMR spectrum of $(R, R, R, R, R, R)-7 \mathbf{b}\left(\mathrm{D}_{2} \mathrm{O}, 75 \mathrm{MHz}\right)$<smiles>CNC1CCCCC1NCCCCCCNC1CCCCC1NCCCCCCNC1CCCC[C@@H]1NC</smiles>

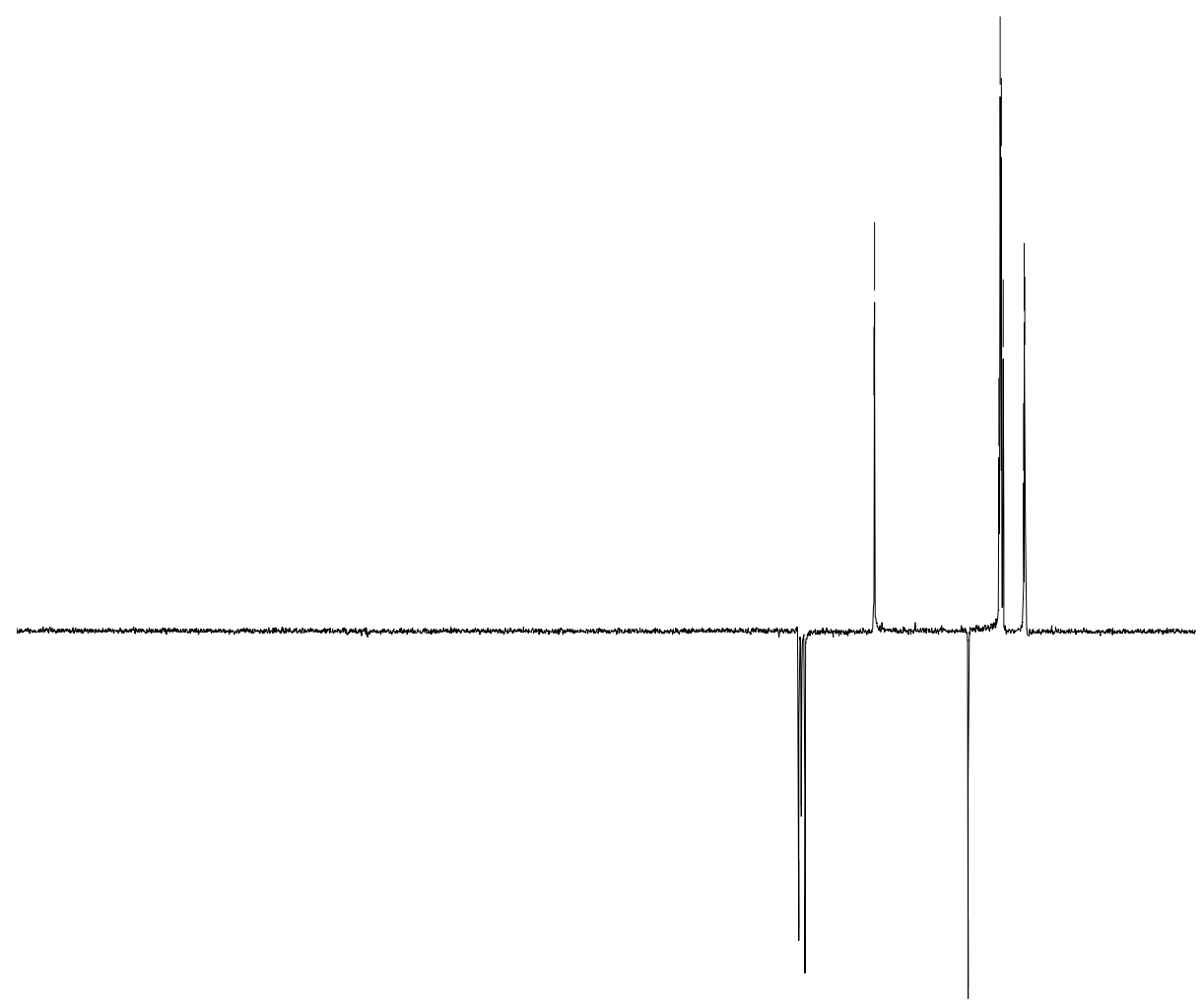

(ppm) 
${ }^{1} \mathrm{H}$ NMR spectrum of $(R, R, R, R, R, R)-8\left(\mathrm{CDCl}_{3}, 300 \mathrm{MHz}\right)$

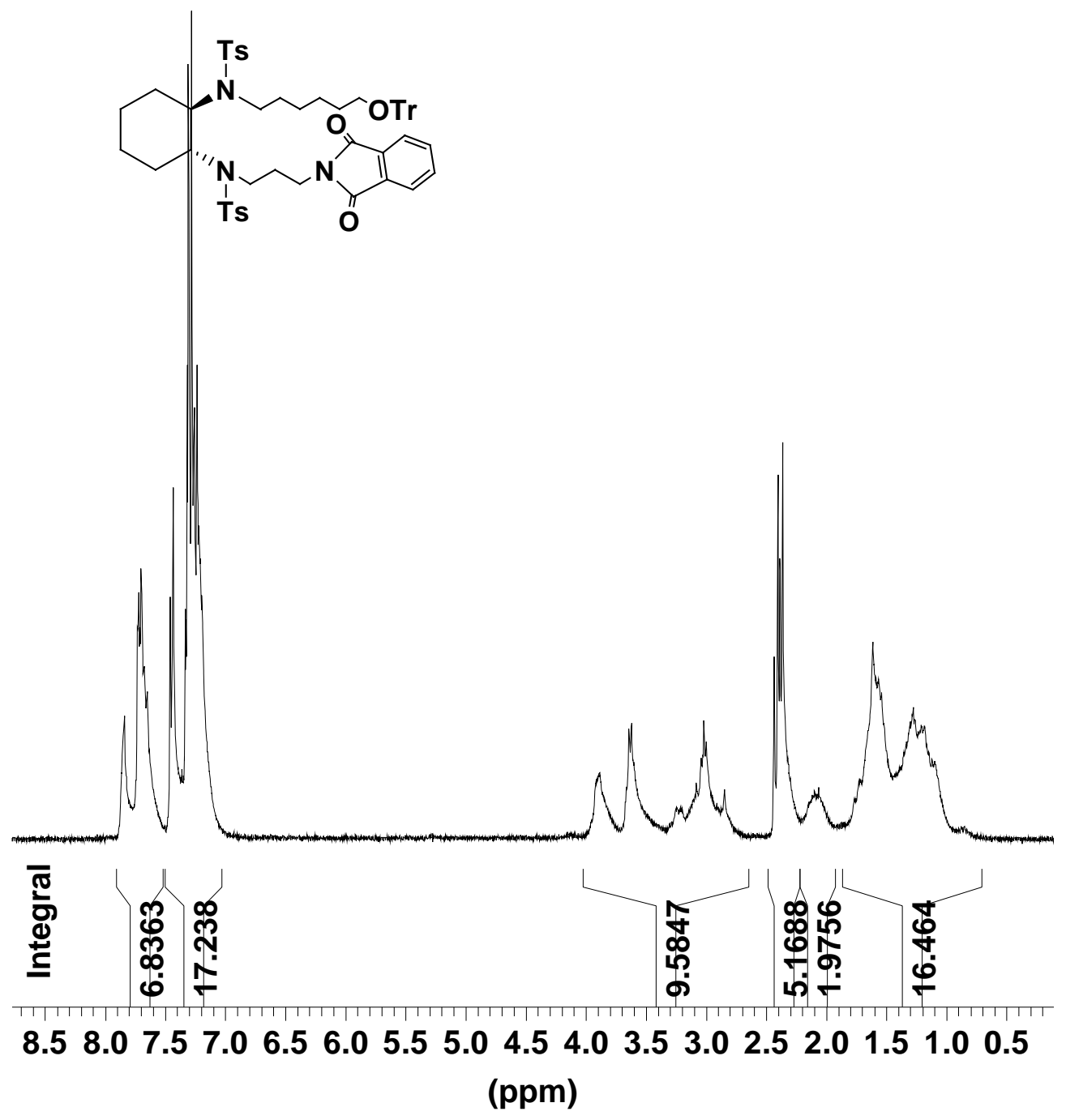


${ }^{13} \mathrm{C}$ NMR spectrum of $(R, R, R, R, R, R)-8\left(\mathrm{CDCl}_{3}, 75 \mathrm{MHz}\right)$

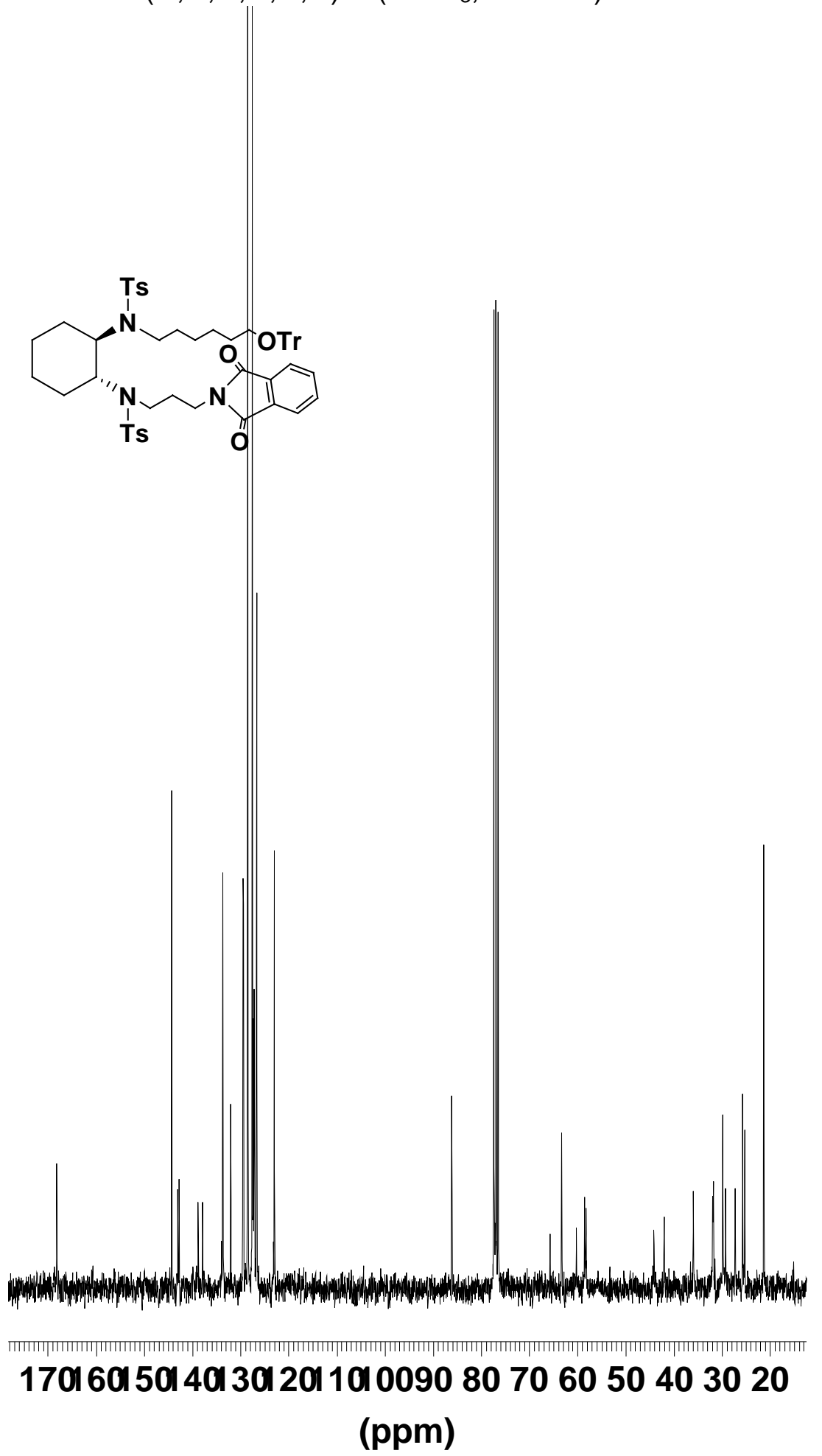

S-48 
DEPT spectrum of $(R, R, R, R, R, R)-8\left(\mathrm{CDCl}_{3}, 75 \mathrm{MHz}\right)$

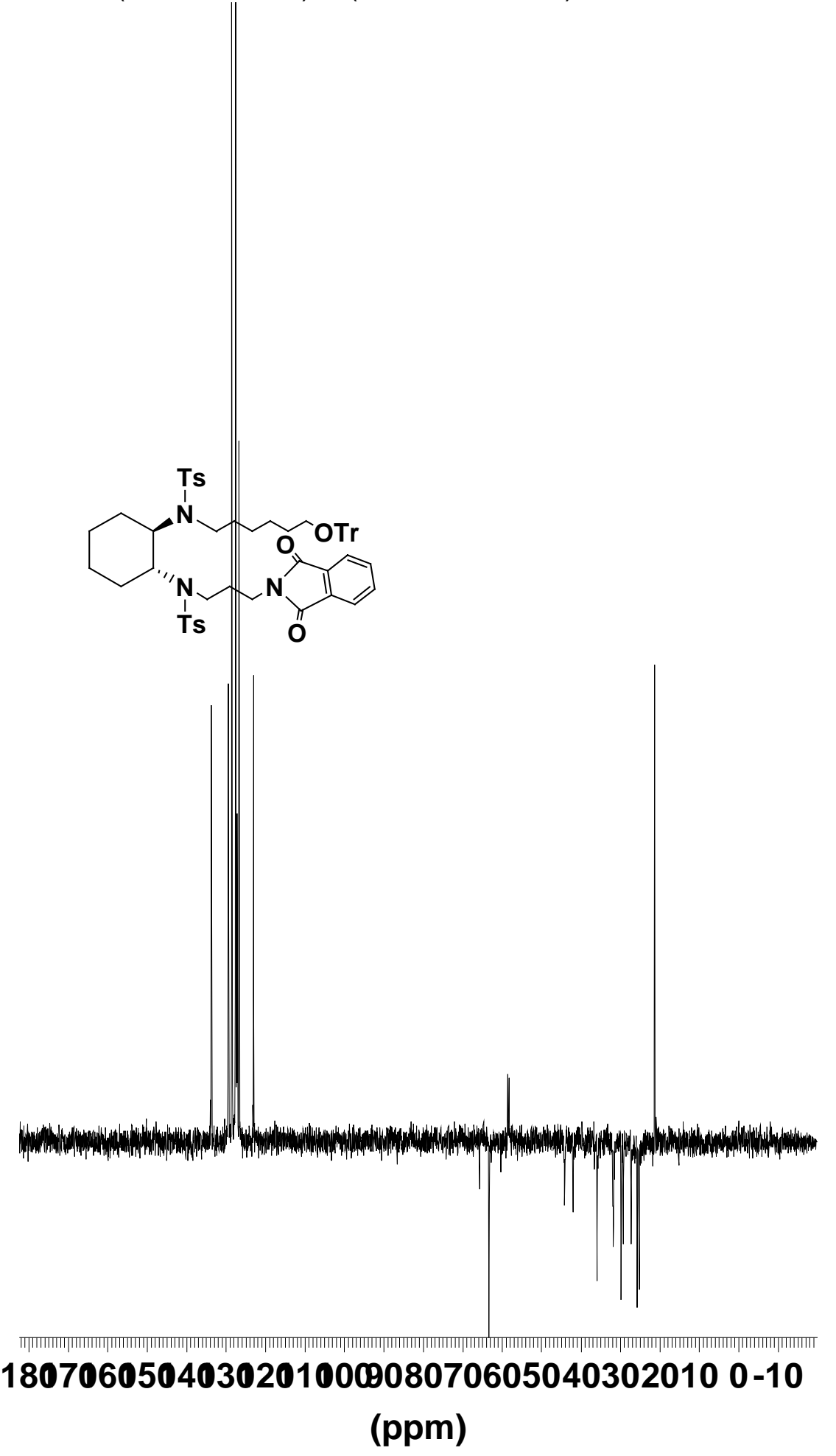


${ }^{1} \mathrm{H}$ NMR spectrum of $(R, R, R, R, R, R)-9\left(\mathrm{CDCl}_{3}, 300 \mathrm{MHz}\right)$
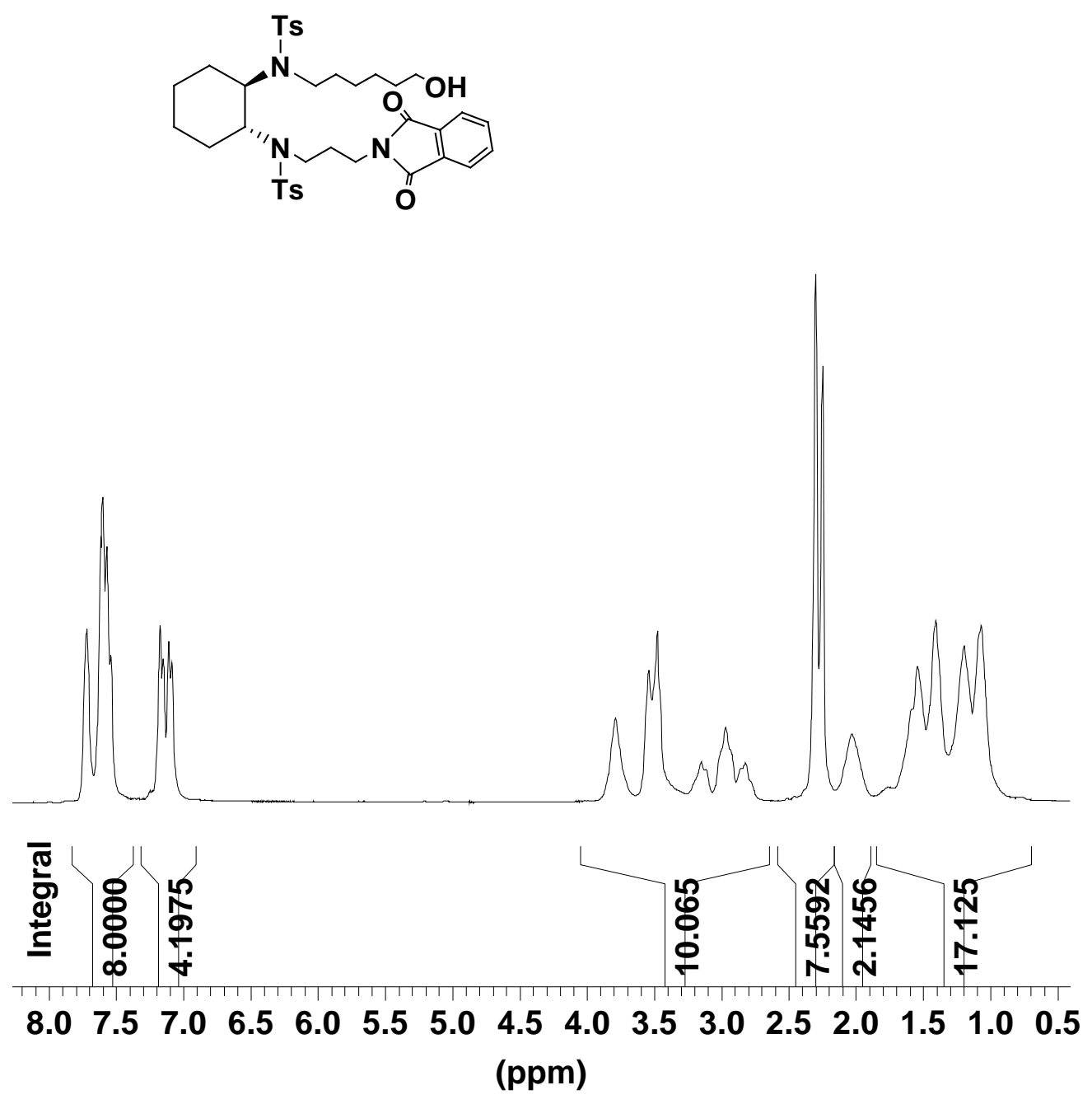
${ }^{13} \mathrm{C}$ NMR spectrum of $(R, R, R, R, R, R)-9\left(\mathrm{CDCl}_{3}, 75 \mathrm{MHz}\right)$
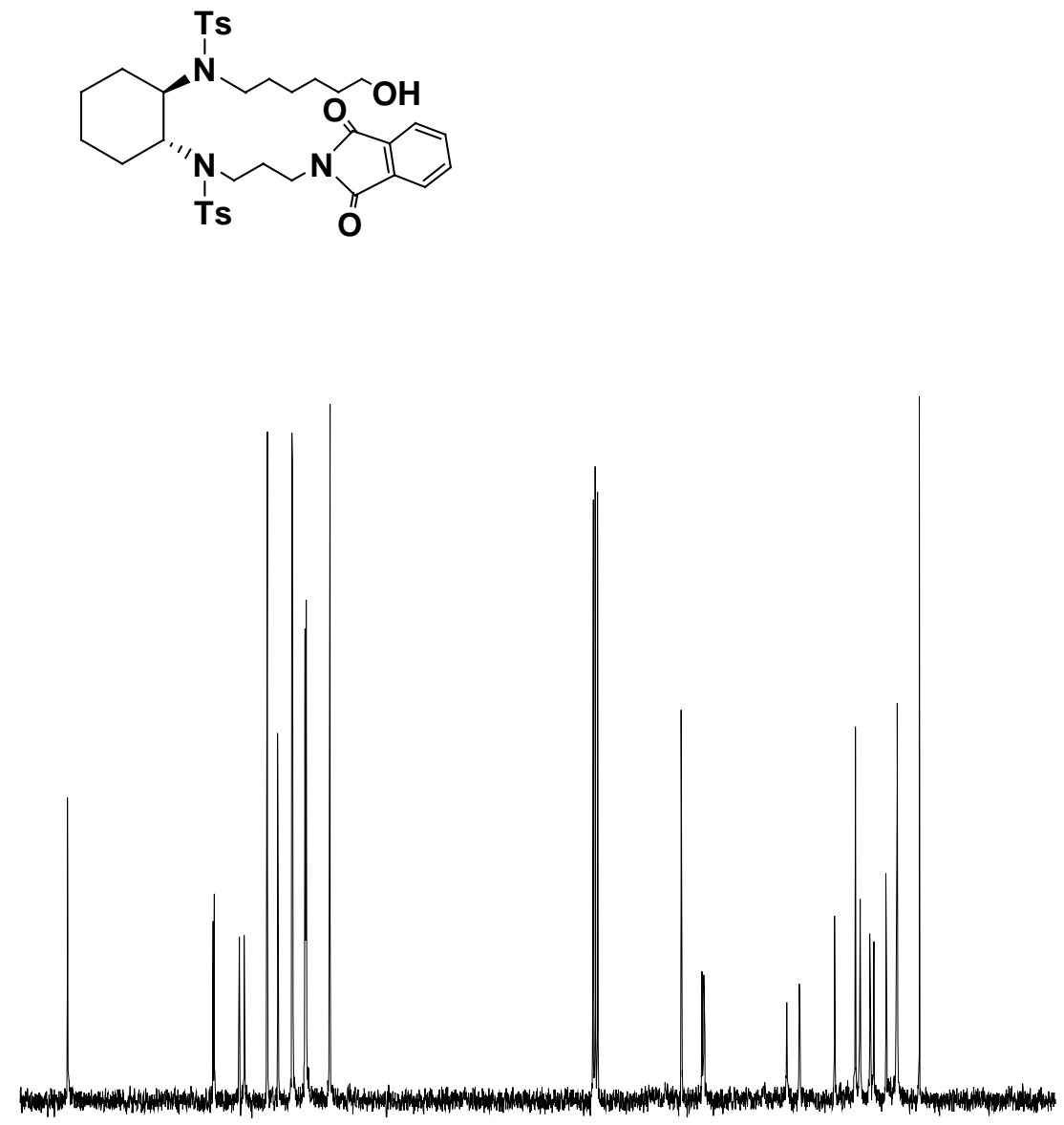

170605040302010009080706050403020100 (ppm) 
DEPT spectrum of $(R, R, R, R, R, R)-9\left(\mathrm{CDCl}_{3}, 75 \mathrm{MHz}\right)$

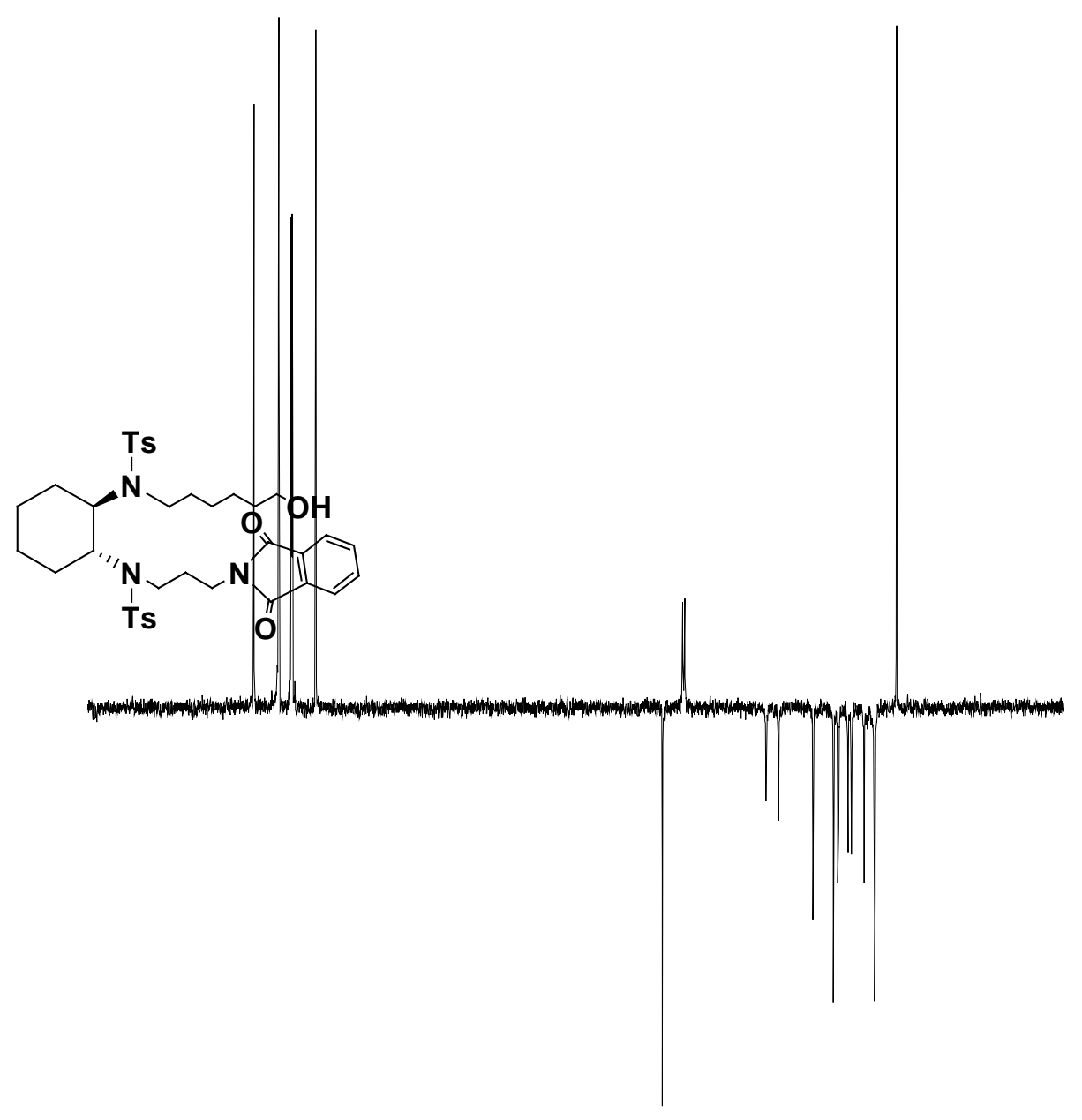

1601501401301201101009080706050403020100

(ppm)

S-52 
${ }^{1} \mathrm{H}$ NMR spectrum of $(R, R, R, R, R, R)-10\left(\mathrm{CDCl}_{3}, 300 \mathrm{MHz}\right)$

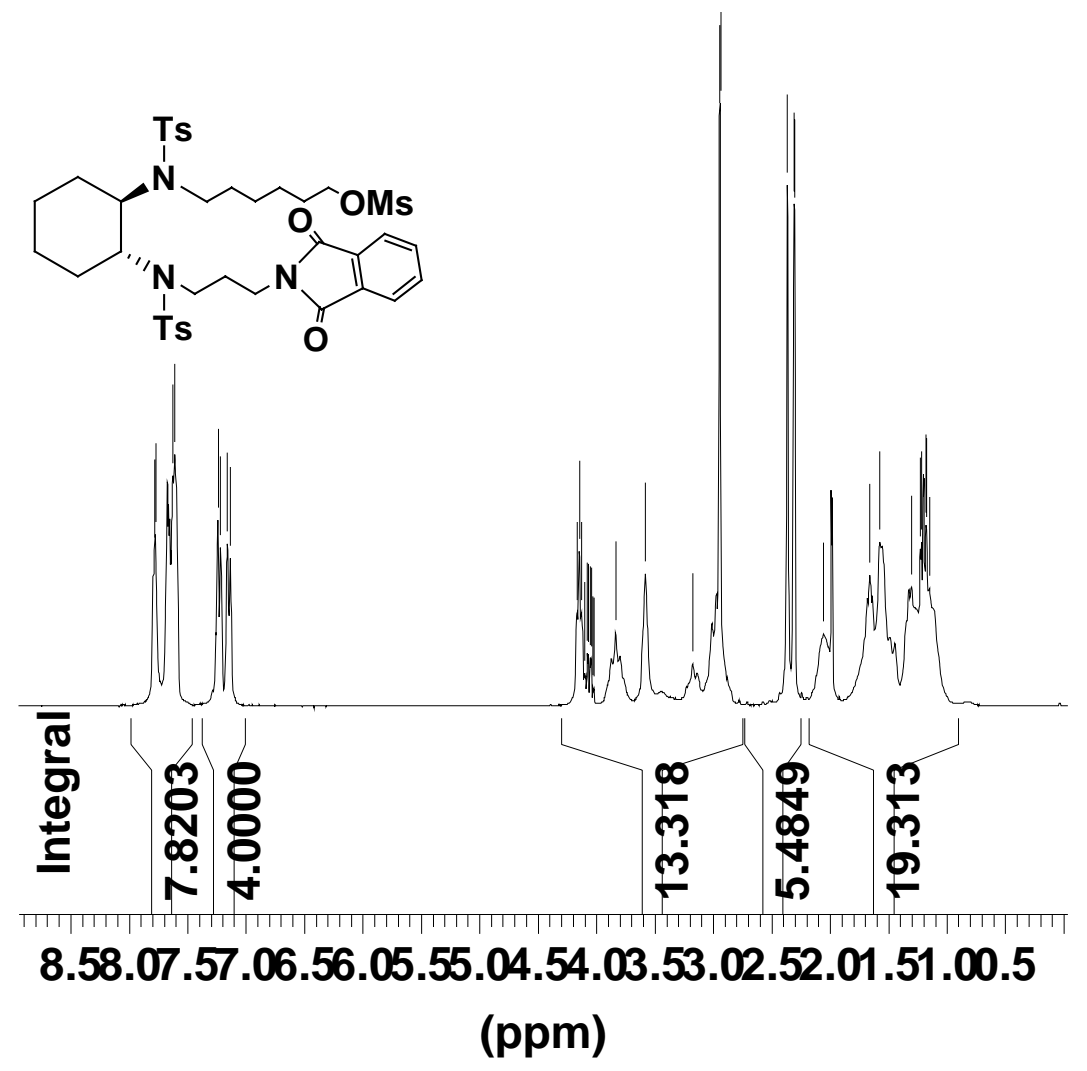

S-53 
${ }^{13} \mathrm{C}$ NMR spectrum of $(R, R, R, R, R, R)-10\left(\mathrm{CDCl}_{3}, 75 \mathrm{MHz}\right)$

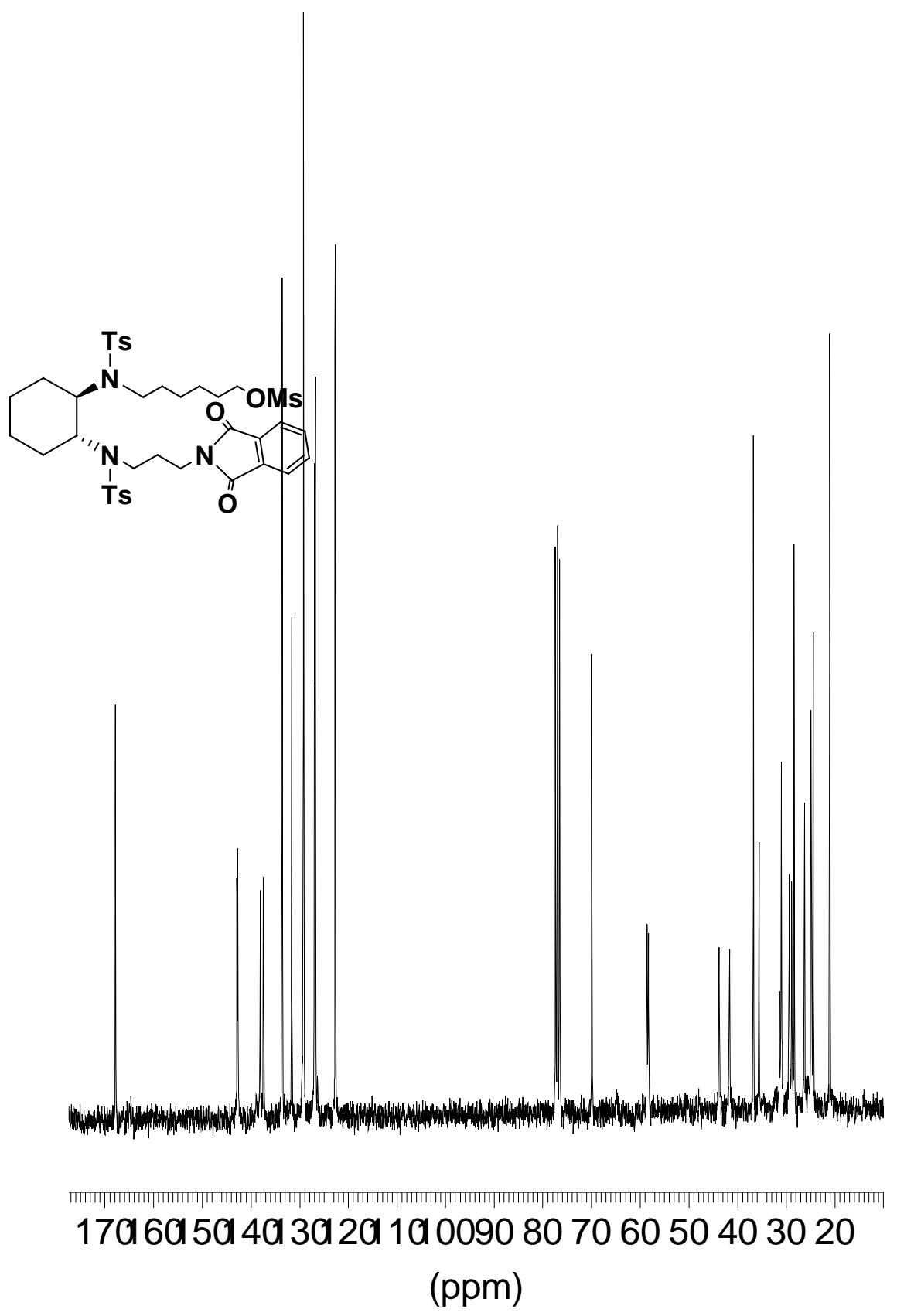

S-54 
DEPT spectrum of $(R, R, R, R, R, R)-10\left(\mathrm{CDCl}_{3}, 75 \mathrm{MHz}\right)$

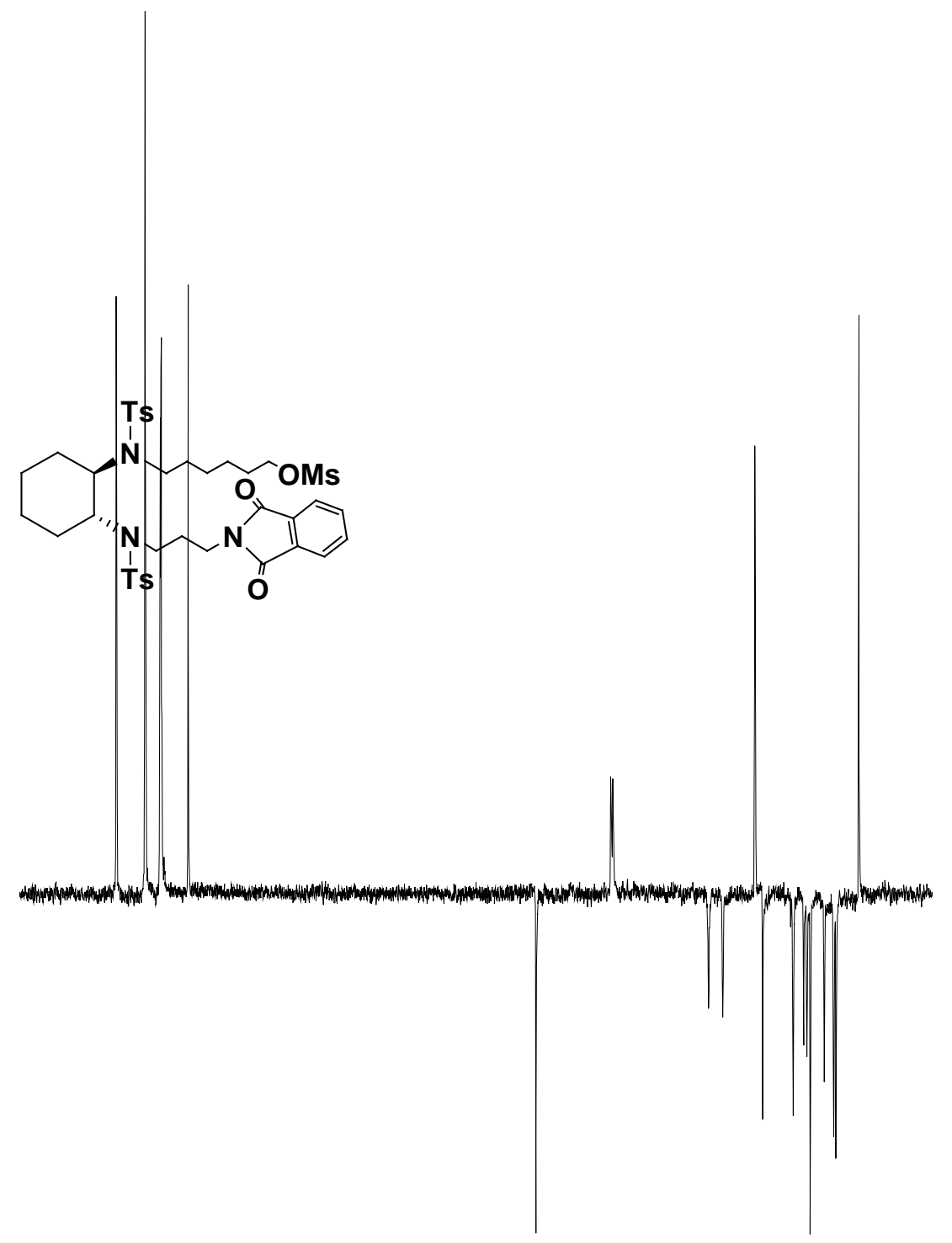

\section{$1401301201101009080706050 \quad 403020$ (ppm)}


${ }^{1} \mathrm{H}$ NMR spectrum of $(R, R, R, R, R, R)-11\left(\mathrm{CDCl}_{3}, 300 \mathrm{MHz}\right)$

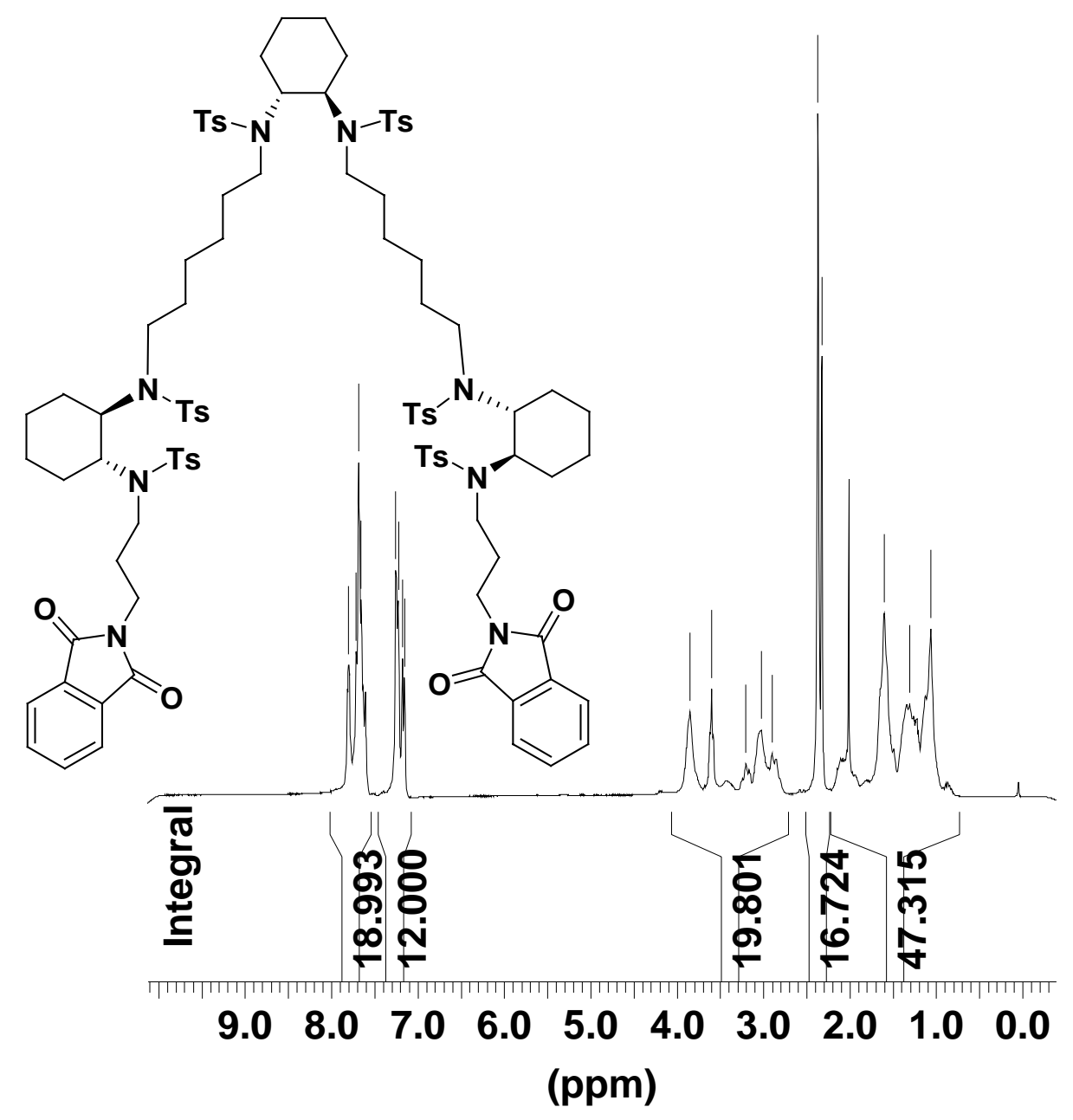


${ }^{13} \mathrm{C}$ NMR spectrum of $(R, R, R, R, R, R)-11\left(\mathrm{CDCl}_{3}, 75 \mathrm{MHz}\right)$

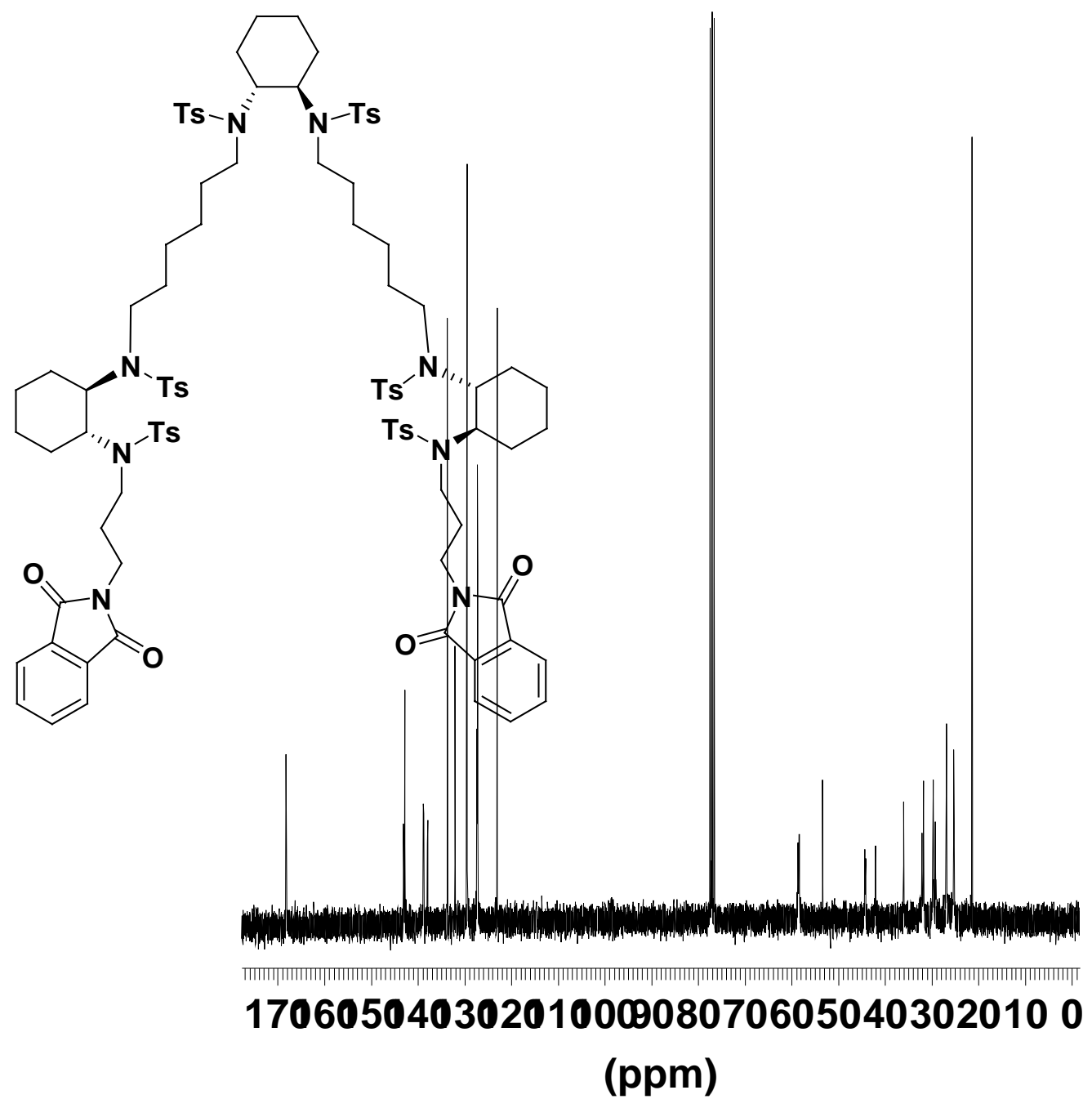


DEPT spectrum of $(R, R, R, R, R, R)-11\left(\mathrm{CDCl}_{3}, 75 \mathrm{MHz}\right)$

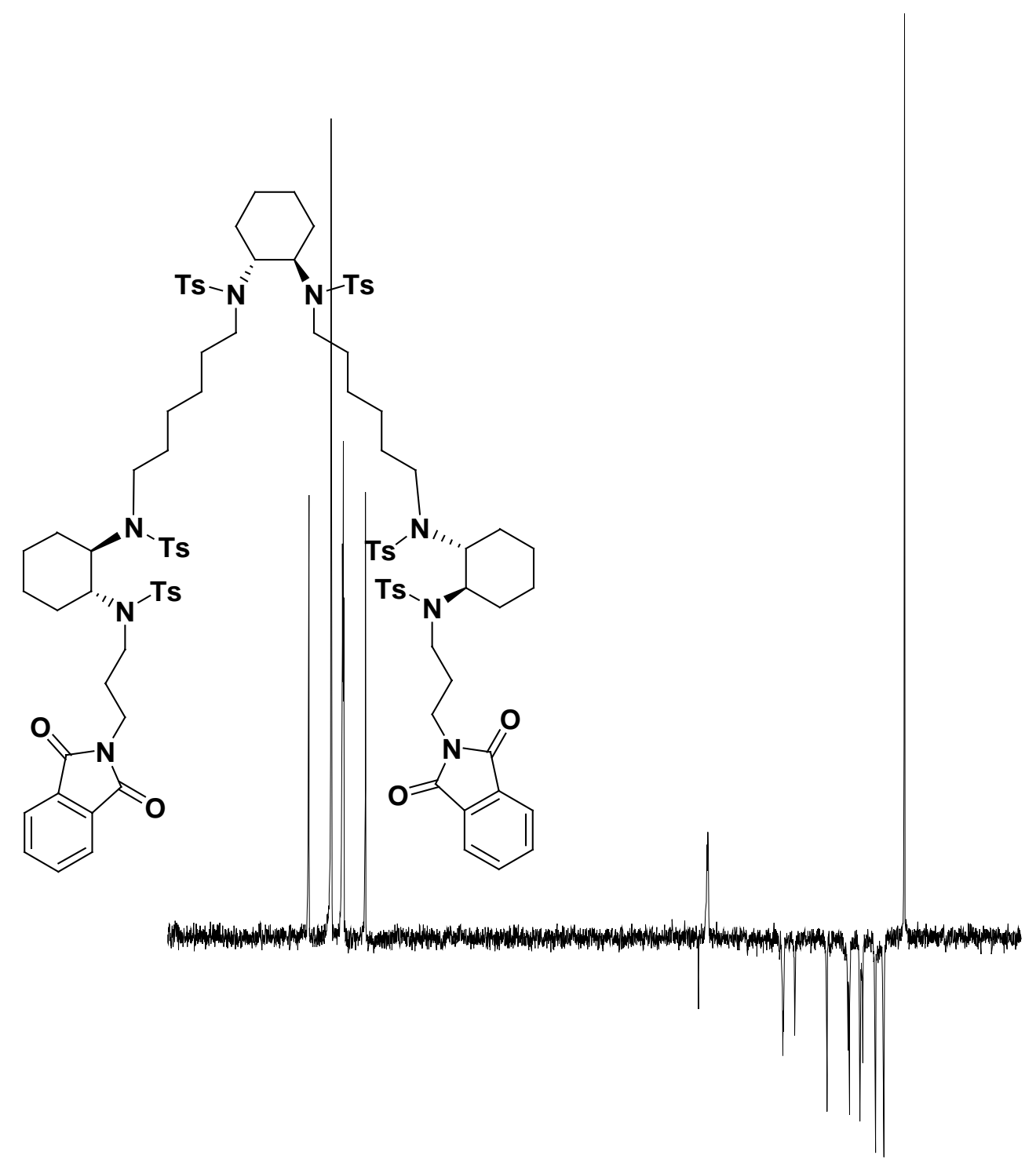

1501401301201101009080706050403020100 (ppm) 
${ }^{1} \mathrm{H}$ NMR spectrum of $(R, R, R, R, R, R)-12\left(\mathrm{CDCl}_{3}, 300 \mathrm{MHz}\right)$

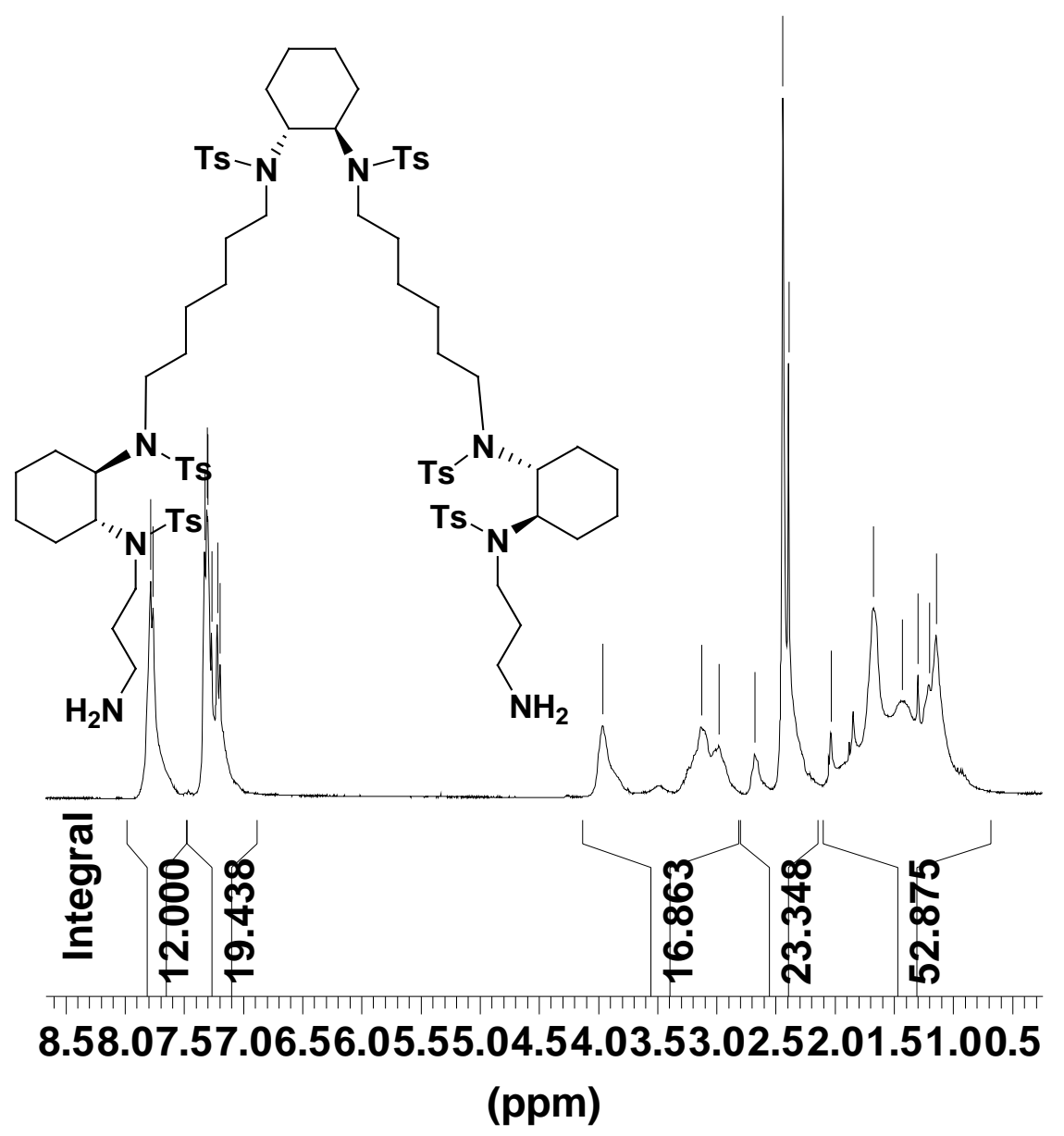


${ }^{13} \mathrm{C}$ NMR spectrum of $(R, R, R, R, R, R)-12\left(\mathrm{CDCl}_{3}, 75 \mathrm{MHz}\right)$

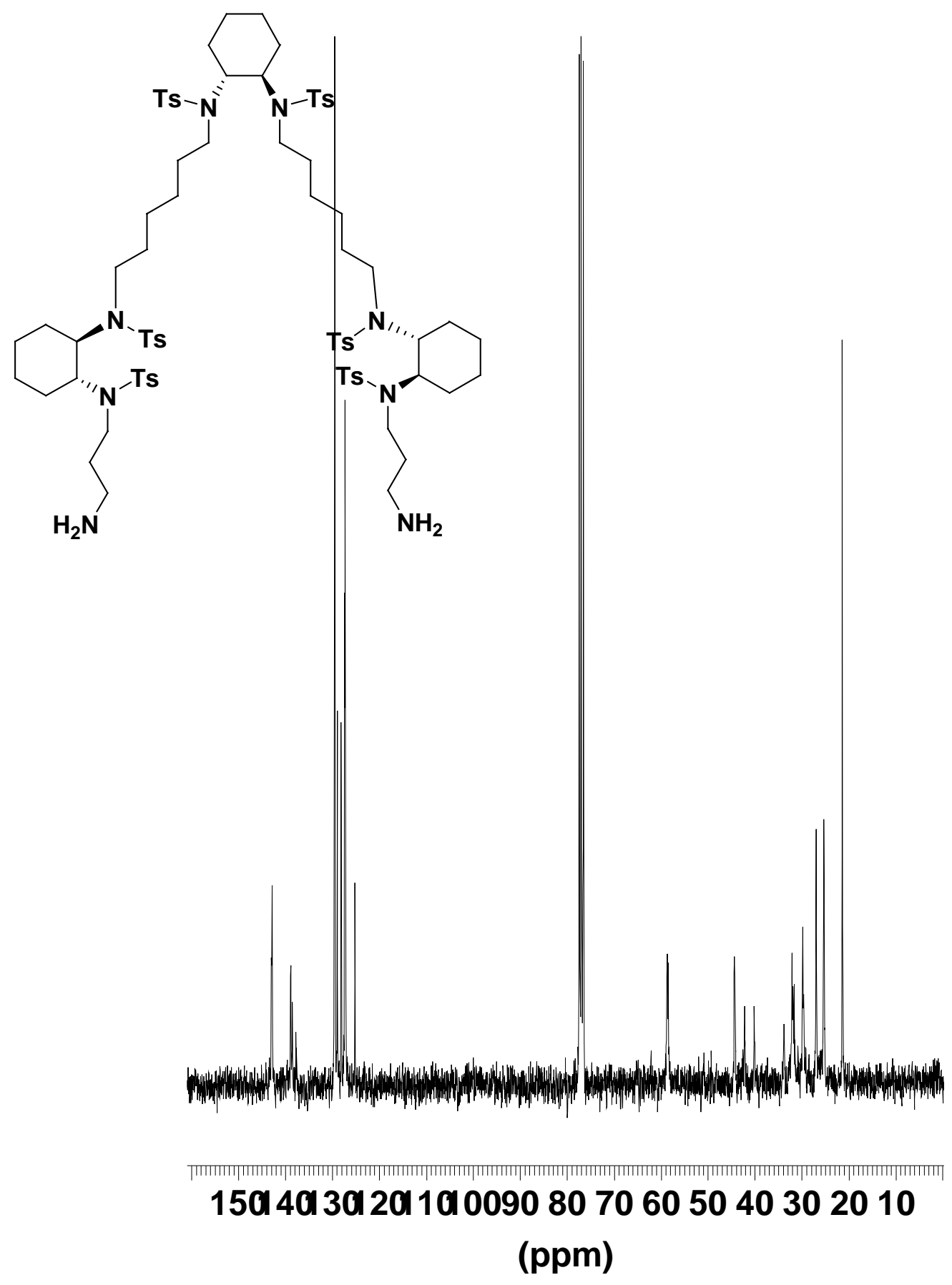

S-60 
DEPT spectrum of $(R, R, R, R, R, R)-12\left(\mathrm{CDCl}_{3}, 75 \mathrm{MHz}\right)$

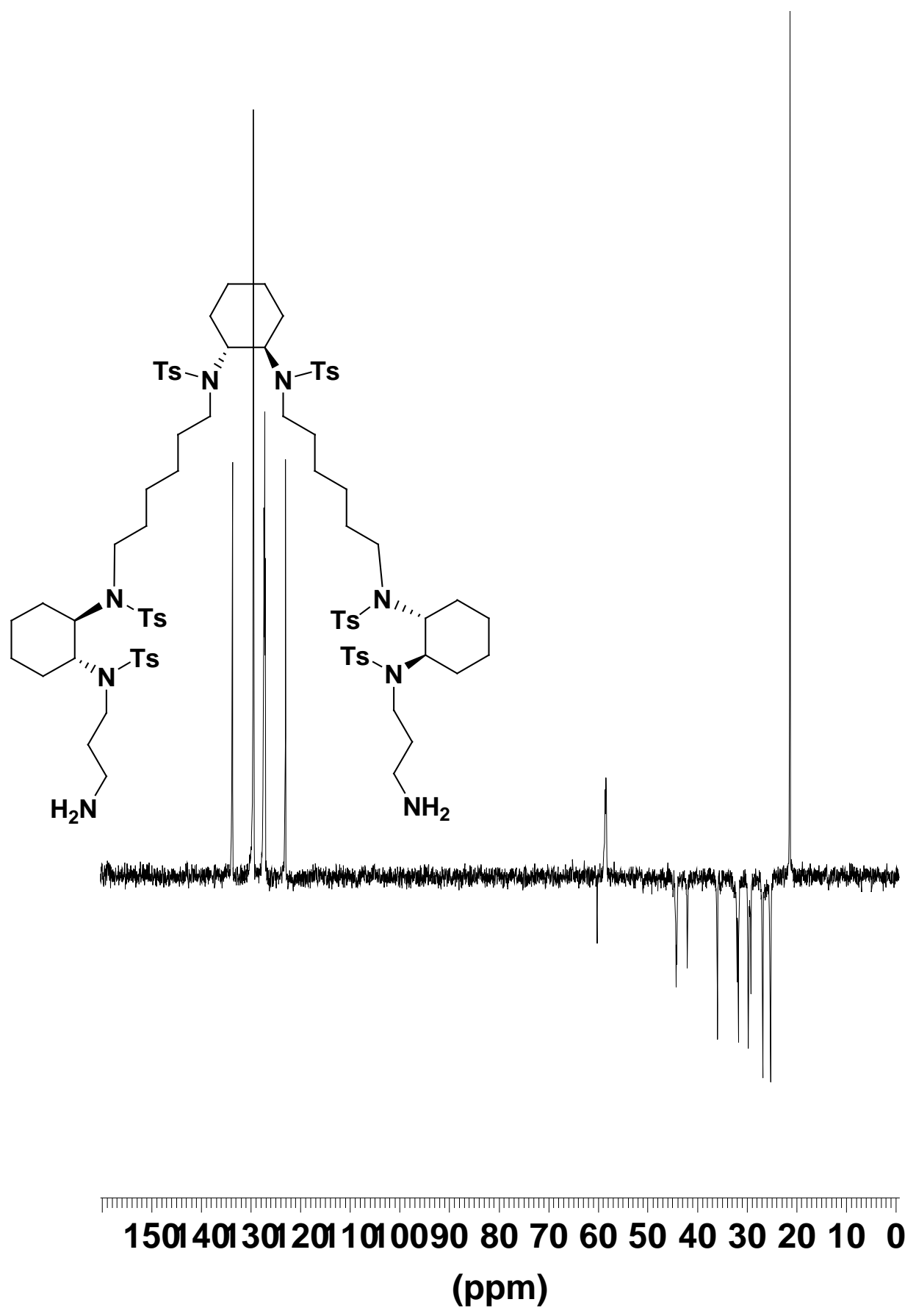


${ }^{1} \mathrm{H}$ NMR spectrum of $(R, R, R, R, R, R)-13\left(\mathrm{D}_{2} \mathrm{O}, 300 \mathrm{MHz}\right)$

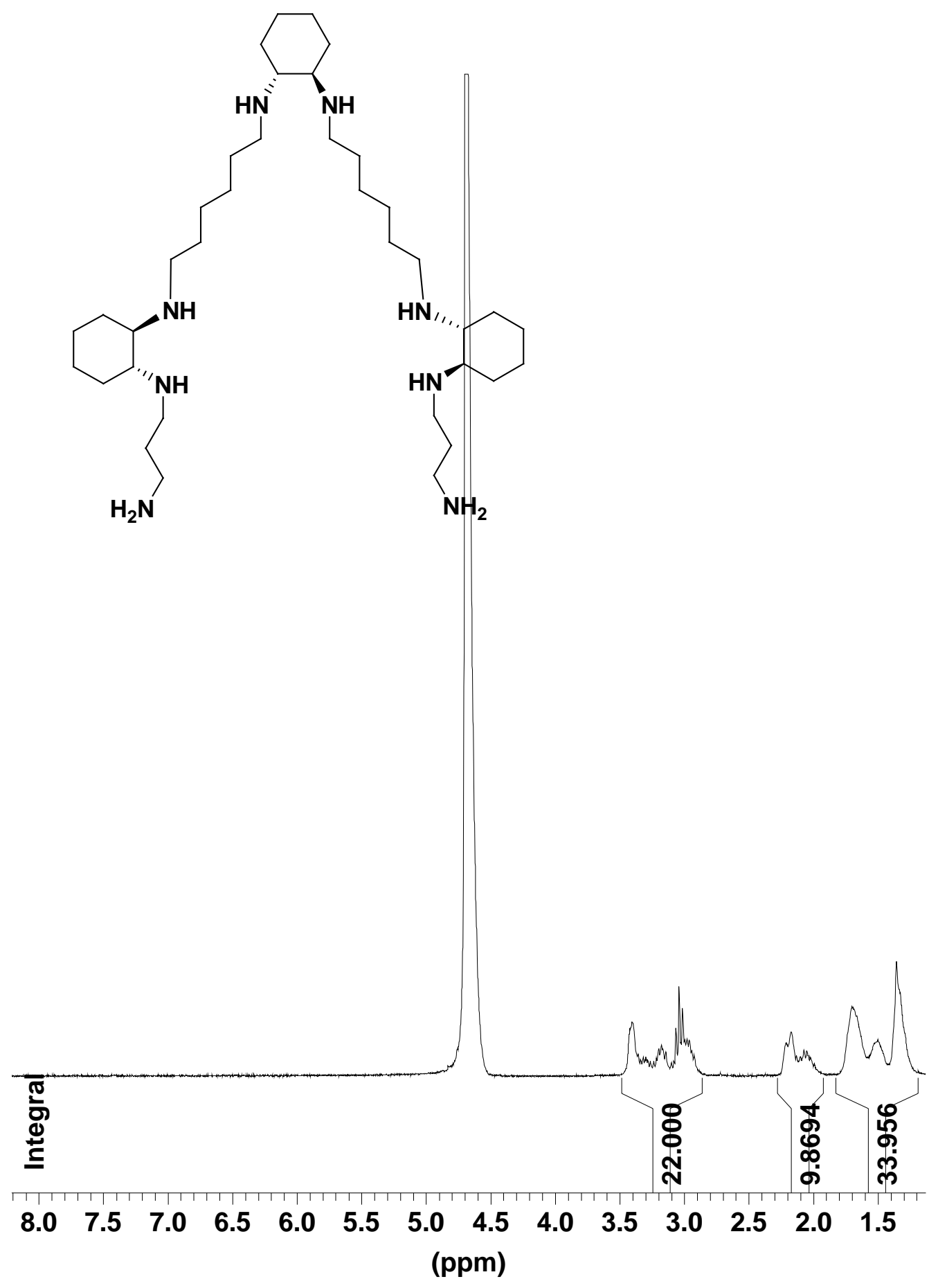


${ }^{13} \mathrm{C}$ NMR spectrum of $(R, R, R, R, R, R)-13\left(\mathrm{D}_{2} \mathrm{O}, 75 \mathrm{MHz}\right)$

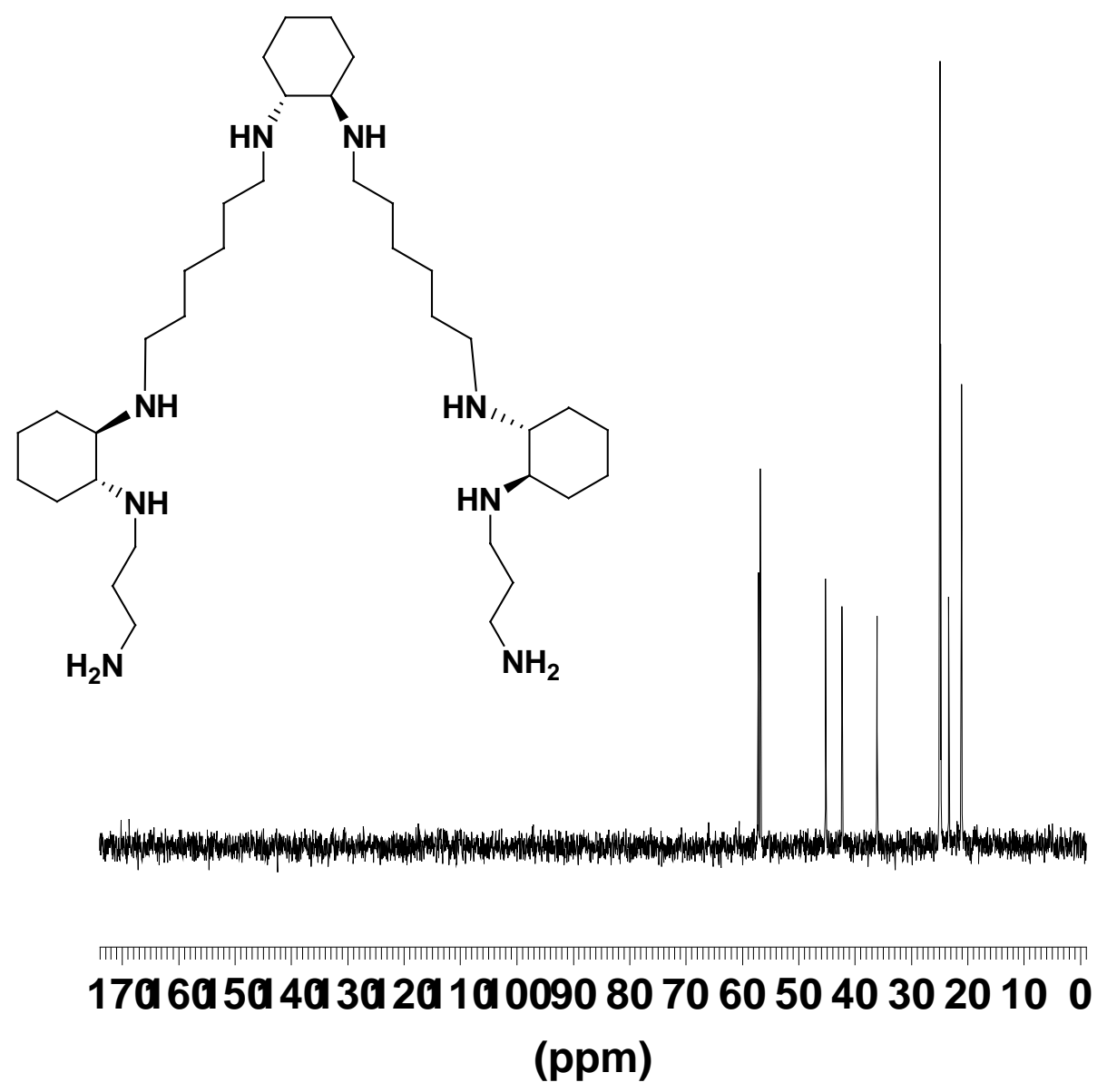


DEPT NMR spectrum of $(R, R, R, R, R, R)-\mathbf{1 3}\left(\mathrm{D}_{2} \mathrm{O}, 75 \mathrm{MHz}\right)$

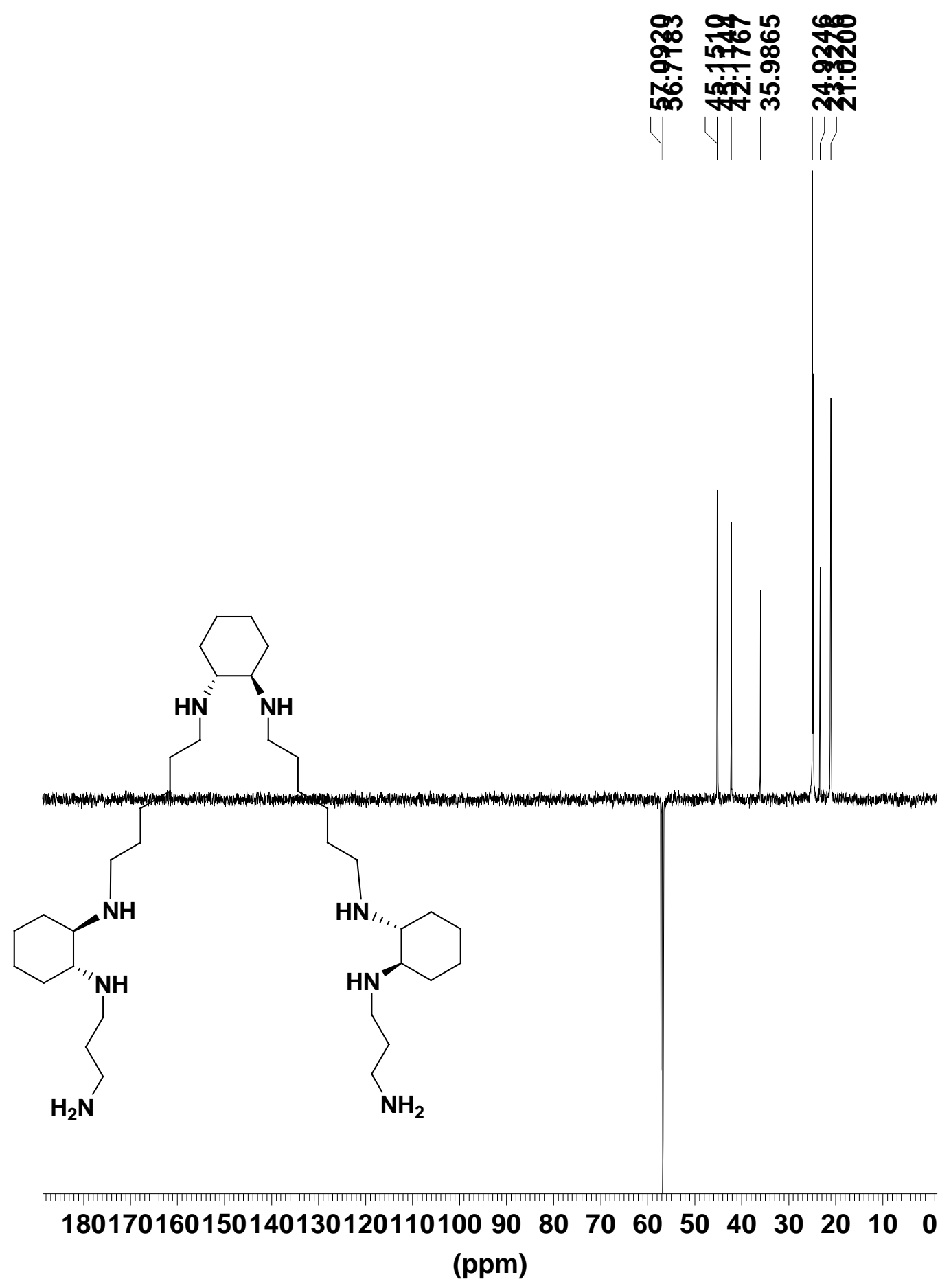

